Portland State University

PDXScholar

10-24-2007

\title{
"Questions About Stuff You Don't Normally See on a Map:" A Study of Sixth-Graders' Abilities to Understand Quantitative Thematic Maps
}

Stephanie Lynn Gaspers

Portland State University

Follow this and additional works at: https://pdxscholar.library.pdx.edu/open_access_etds

Part of the Geography Commons

Let us know how access to this document benefits you.

\section{Recommended Citation}

Gaspers, Stephanie Lynn, "'Questions About Stuff You Don't Normally See on a Map:" A Study of SixthGraders' Abilities to Understand Quantitative Thematic Maps" (2007). Dissertations and Theses. Paper 2425.

https://doi.org/10.15760/etd.2422

This Thesis is brought to you for free and open access. It has been accepted for inclusion in Dissertations and Theses by an authorized administrator of PDXScholar. Please contact us if we can make this document more accessible: pdxscholar@pdx.edu. 


\section{THESIS APPROVAL}

The abstract and thesis of Stephanie Lynn Gaspers for the Master of Science in Geography were presented October 24, 2007, and accepted by the thesis committee and the department.

COMMITTEE APPROVALS:

Jdseph Poracsky, Chair

Teresa L. Bulman

David Banis

Thomas Kindermann

Representative of the Office of Graduate Studies

DEPARTMENT APPROVAL:

Martha A. Works, Chair

Department of Geography 
ABSTRACT

An abstract of the thesis of Stephanie Lynn Gaspers for the Master of Science in Geography presented October 24, 2007.

Title: "Questions About Stuff You Don't Normally See on a Map:" A Study of Sixth- Graders' Abilities to Understand Quantitative Thematic Maps

Middle school students work with many types of maps in school, however most maps they use are qualitative thematic maps that only show differences in kind as compared to quantitative thematic maps that show differences in amounts. This thesis investigates sixth-grade students' abilities to analyze three types of thematic maps: dot maps, choropleth maps, and graduated circle maps. Two hundred and two Oregon sixth-graders were tested on their abilities to interpret map symbology, make inferences from the data, categorize values into regions, and ask geographic questions concerning data distributions. The results indicate that students can understand these three quantitative thematic maps for these purposes. These results also raise the question, "Why aren't there more quantitative thematic maps presented to students in middle school curriculum?" Key words: thematic maps, children's cartography, geographic education. 
"QUESTIONS ABOU1' STUFF YOU DON'T NORMALLY SEE ON A MAP:" A STUDY OF SIXTH-GRADERS' ABILITIES TO UNDERSTAND QUANTITATIVE THEMATIC MAPS

\author{
by \\ STEPHLANIE LYNN GASPERS
}

A thesis submitted in partial fulfillment of the

requirements for the degree of

\author{
MASTER OF SCLENCE \\ in \\ GEOGRAPHY
}

Portland State University

2007 
To Joe, it's been an honor 


\section{ACKNOWLEDGMENTS}

When I first started the graduate program, I wasn't quite sure where I was headed. However, with the help of countless people here at Portland State University, I have found my niche in geography. I am forever indebted to Dr. Joe Poracsky. Without his patience, hospitality, guidance, and most importantly—his encouragement-I would not have finished this thesis. Surely, I thought revisions to the thesis drafts would never end as I tried to meet his standards, but I am grateful for the time he invested in my endeavors. He is truly a mentor in every sense of the word and has passed onto me his immense knowledge of traditional cartography that is seemingly beginning to disappear in the war between cartography and GIS.

Thanks to my committee members for their assistance that enabled me to unite cartography and geography education for my research. Dr. Teresa Bulman was the link to the Oregon Geographic Alliance Teachers. Additionally, her research agenda has given me opportunities to improve my cartographic skills for use in geography education. David Banis provided many cartographic experiences and was there to laugh with me at the interesting things that present themselves throughout the production stages. We have spent many hours critiquing different cartographic techniques that have enhanced my understanding of cartography for children. I must also thank Dr. Thomas Kindermann, whom I was first introduced to as he curiously wanted to know what a geographer was doing in his psychology class. After sharing my research ideas with him, he has 
only kind and encouraging words to send my way and even asked if he could serve on my committee. I am pretty sure that is the first recorded incidence of a professor asking to be part of a thesis committee to take on extra work, but the fact that he did illustrates to me that this thesis means something outside of geography. His excitement has been a source of encouragement.

Thanks also to those who allowed the actual field study to be conducted. Four sixth-grade teachers welcomed me into their classrooms to work with their wonderfully curious students. Obviously, without their willingness to participate, there would be no study to conduct. Additionally, my helpers: Jenny McKay, Joel Stewart, and Joe Poracsky gave up a day in their busy schedules to assist me in the classrooms answering questions from students.

Thank you to the funding sources that made this study possible. The Association of the American Geographers Cartography Specialty Group and PSU's Chapter of the American Society of Photogrammetry and Remote Sensing provided research grants and the PSU Geography Department awarded the John Rockie Scholarship to help fund this study. Graduate student salaries are not conducive to making color copies of these maps and this funding has been essential to the completion of this research.

I must thank those who guide the department and thus directly impact my graduate experience. Karin Waller has made the bureaucracy of graduate school fairly painless. She is always there to remind us of deadlines, take care of way more paperwork than I care to think about, and most importantly, be there to 
offer a friendly smile. Additionally, Martha Works keeps all of us in the department relatively happy (which isn't an easy task!) by offering her friendly assistance and expertise. I appreciate her undying concern, support, and kindness apparent in all that she does.

Finally, I send the most heart-felt thank you to all of you who were part of the PSU geography department while I was here (especially the unforgettable characters that comprised my cohort). Without the laughs, the tears, and the amazing times together in this beautiful city, I would not have completed the program and would definitely not be the person I am today. Unfortunately, the English language affords me few other options besides the words "thank you" to communicate my gratitude in this short acknowledgment and thus I can only hope that along the way I've shown my appreciation to the aforementioned people. 


\section{TABLE OF CONTENTS}

ACKNOWLEDGEMENTS .ii

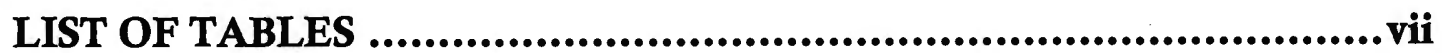

LIST OF FIGURES...............................................................viii

1. INTRODUCTION ........................................................1

Standards for Geography Curriculum ..............................................2

Map Types and Use ......................................................................4

Review of Maps Used in Geography Curriculum ............................9

Research Objectives and Hypotheses .............................................11

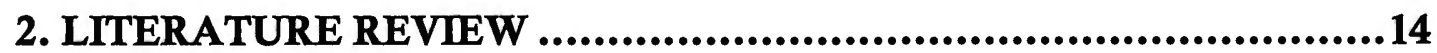

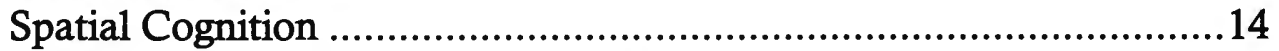

Piaget's Theory of Cognitive Development .....................................15

Vygotsky's Sociocultural Theory of Cognitive Development..............19

Cognitive Processes Critical to Thematic Maps ..................................220

Symbology Understanding ..................................................21

Ability to Compare and Contrast ............................................23

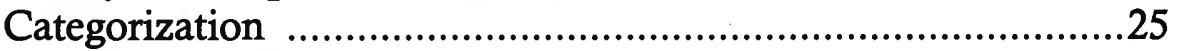

Cognitive Abilities Directly Tied to Cartography .......................27

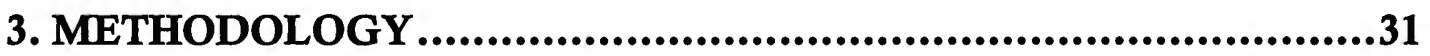

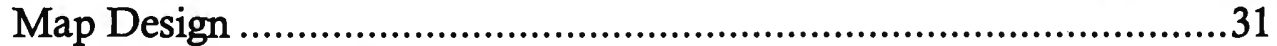

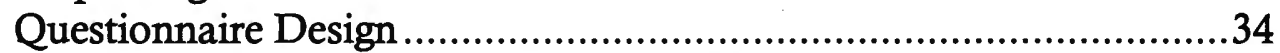

Questions 1 and 3: Interpreting the Symbolization ....................35

Question 2: Making Inferences ..............................................35

Question 4: Spatial Pattern Recognition....................................36

Question 5: Spatial Relationships and Inquiry ............................37

Question 6: Symbolization Understanding ..............................38

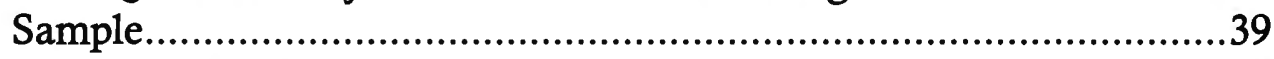

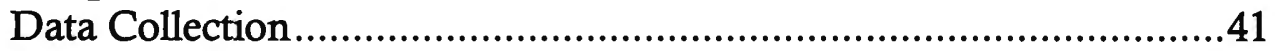

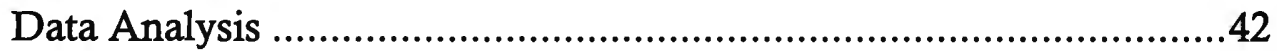

4. RESULTS................................................................44

Questions 1 and 3: Interpreting the Symbolization ..........................46

Question 2: Making Inferences .................................................53

Question 4: Spatial Pattern Recognition........................................56

Question 5: Thinking Geographically ...........................................59

Question 6: Understanding the Symbolization...................................63 
Student Demographic Characteristics ..........................................66

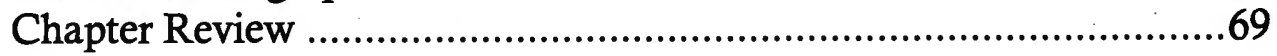

5. ANALYSIS AND DISCUSSION .........................................71

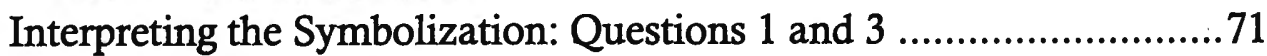

Making Inferences: Question 2 ...................................................76

Spatial Pattern Recognition: Question 4 ........................................8 80

Thinking Geographically: Question 5 ............................................89

Understanding the Symbolization: Question 6...............................91

Overall Performance ................................................................92

Student Demographic Characteristics ..........................................99

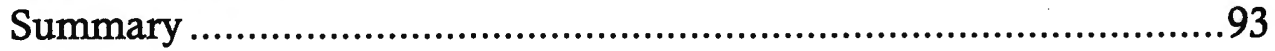

6. RECOMMENDATIONS AND CONCLUSIONS .........................95

WORKS CITED............................................................103

APPENDIX A. Middle School Social Studies Textbooks........................106

APPENDIX B. Map Creation Methodology ...................................107

APPENDIX C. Map Surveys .................................................115

APPENDIX D. Human Resources Approval ...................................128

APPENDIX E. Script.......................................................129 


\section{LIST OF TABLES}

Table 1. Results of middle school textbook survey ........................................10

Table 2. Young's results of student's map reading .......................................28

Table 3. Comparison of thematic maps.........................................................32

Table 4. Participating sixth-grade classes ....................................................39

Table 5. Completion time summary statistics ...............................................45

Table 6. Chi-square contingency table for Question 1 ....................................51

Table 7. Chi-square contingency table for Question 3 ...................................53

Table 8. Chi-square contingency table for Question 2.................................55

Table 9. Chi-square contingency table for Question 4...................................59

Table 10. Responses to Question 5 ..........................................................61

Table 11. Chi-square contingency table for Question 5 .................................62

Table 12. Chi-square contingency table for Question $6 \ldots \ldots \ldots \ldots \ldots \ldots \ldots \ldots \ldots \ldots \ldots . . . . . .65$

Table 13. Overall performance by map group .............................................67

Table 14. Chi-square contingency table for overall scores ...............................68

Table 15. Chi-square values of characteristics and overall performance...........68

Table 16. Percentage of students who recorded lowest value in key as answer.74

Table 17. Percentage of students who marked Multnomah County ................77

Table 18. Chi-square contingency table for Multnomah County answers .........78

Table 19. Chi-square contingency table for answers that labeled each county..83

Table 20. Chi-square contingency table for boundaries that follow counties ....86

Table 21. Summary statistics for number of regions drawn ............................87

Table 22. Types of correct questions asked...................................................90

Table 23. Hypothesis overview .................................................................94 


\section{LIST OF FIGURES}

Figure 1. Example of a general reference map.............................................5

Figure 2. Example of a thematic map …................................................

Figure 3. Thematic map symbol types ......................................................

Figure 4. Levels of map reading skills .....................................................12

Figure 5. Approximate ages corresponding with Piaget's stages.....................16

Figure 6. Examples of conservation problems ................................................18

Figure 7. Choropleth map depciting population density ..............................24

Figure 8. Graduated circle map depicting county population...........................26

Figure 9. Number of each gender that participated ........................................40

Figure 10. Age of participants .............................................................40

Figure 11. Box and whisker plots of map completion time ...........................45

Figure 12. Distribution of answers given for Question 1 on choropleth map....49

Figure 13. Distribution of answers given for Question 1 on dot map...............49

Figure 14. Distribution of answers given for Question 1 on graduated circle ...49

Figure 15. Correct answers to Question 1 .............................................50

Figure 16. Correct answers to Question 3 ................................................52

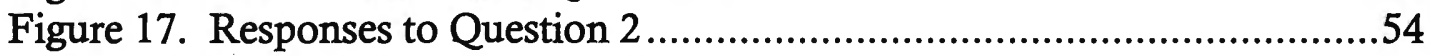

Figure 18. Performance on Question 2 ....................................................55

Figure 19. Examples of Question 4 answers...........................................57

Figure 20. Correct responses to Question 4 ..............................................58

Figure 21. Percentage of correct responses to Question 5...............................61

Figure 22. Percentage of correct responses to Question 6...............................65

Figure 23. Overall performance by map group .............................................66

Figure 24. Range of map values covered by each map legend ........................73

Figure 25. Lowest value in each map key .....................................................74

Figure 26. Examples of county labeling .....................................................81

Figure 27. Percentage of incorrect answers that only had counties labeled ......82

Figure 28. Examples from students illustrating lines on borders.....................84

Figure 29. Percentage of correct answers that followed county boundaries......85

Figure 30. Examples of supplemental map keys ...........................................100 


\section{CHAPTER 1:}

\section{INTRODUCTION}

Geographers rely on maps as a primary tool for dissemination of information because maps efficiently show data distributions. Maps present data in a manner that enables people to visualize spatial distributions and identify spatial patterns (Sauer 1956). Once map readers identify patterns, they can begin to ask questions such as "Why are things located there?" and "What significance does this distribution have for other phenomena?" Asking and answering such questions helps people acquire knowledge and is an essential skill that geographic educators advocate for as part of school curriculum (Geography Education Standard Project [GESP] 1994).

This skill can be practiced using many types of maps. Traditionally, maps are divided into two broad categories: general reference maps and thematic maps. In the classroom, students most often encounter thematic maps, especially those that illustrate population distributions, climatic patterns, historical events, and economic activity. Thematic maps may be further grouped into qualitative thematic maps, that depict differences in kinds of phenomena, and quantitative thematic maps, that emphasize differences in amounts of phenomena. Although there are many types of both qualitative and quantitative thematic maps available, textbooks rarely employ quantitative thematic maps for use by middle school students.

This thesis will be guided by the following thesis statement: 


\section{If children can understand quantitative thematic maps, then more of these types of maps should be used in classrooms to teach geographic skills instead of primarily relying on qualitative thematic maps.}

To support this argument, this thesis will explore the types of maps middle school students encounter and are expected to use in school. It will describe the cognitive development required to understand spatial patterns and relationships that may help explain the results of this study. It will detail the methods and analysis used to test sixth-grade students' abilities to read and comprehensively understand three types of quantitative thematic maps. Finally, it will discuss the results in a broader context to explain the importance of the results.

\section{Standards for Geography Curriculum}

Curriculum standards are one of the most influential elements of a student's education because they guide what teachers choose to instruct and thus determine what students are expected to use. Curriculum standards in the United States are established separately by each state's education department, resulting in a spectrum of "important" geography concepts taught across the nation. In 1994, as a means to make more consistent standards that covered all aspects of geography, a consortium of geography scholars developed the National Geography Standards to ensure every student becomes a "geographicallyinformed person" (GESP 1994, 29). 
This 1994 document describes eighteen standards, arranged into six overarching themes, that each state can either use or disregard as it wishes. These standards provide descriptions and examples of specific geography subject matter, skills, and perspectives. Three of these eighteen standards explain map skills students should be expected to know, illustrating the importance of maps as tools for geographers (GESP 1994).

The authors of the Oregon Department of Education (ODE) Geography Standards also incorporate map skills into state standards to ensure students are proficient at map reading. By the end of fifth grade, ODE's Geography Content Standard 2 requires students to "Use maps and other geographic tools and technologies to acquire, process, and report information from a spatial perspective" (ODE 2006). To achieve this goal, students will be tested on their abilities to "Examine and understand how to prepare maps, charts, and other visual representations to locate places and interpret geographic information." Additionally, by the time students reach eighth grade, ODE's Content Standard 1 requires them to be able to, "Read, interpret, and understand how to construct geographic representations to analyze information, understand spatial relationships, and compare places" (ODE 2006). To achieve this goal, students will be tested on their ability to "use maps, charts, graphs, and photographs to analyze spatial distributions and patterns" (ODE 2006). Both the National Geography Standards and ODE's Geography Standards emphasize the importance of map skills to geography education. More importantly, the 
standards recognize that students should be competent with a variety of map types to fully understand geographic distributions and patterns.

\section{Map Types and Use}

While stressing variety, the standards fail to set guidelines to ensure students become proficient at reading specific types of maps. In traditional cartographic literature, maps are frequently categorized into two broad groups: general reference maps and thematic maps (Slocum et al. 2005). General reference maps are commonly used for data storage as illustrated in Figure 1. They represent and depict data that actually exist as physical entities and are observable on the ground. Examples of features found on general reference maps include water bodies, roads, schools, and parks. These types of maps are the first students encounter in school and are conventionally used to teach them scale, distance, direction, and navigation skills (e.g. Downs et al. 1988, Blades et al. 1995, Sowden et al. 1996, Leinhaardt et al. 1998). Most academic research on children's cartography employs general reference maps and focuses on how well children understand them for navigational purposes. Children's performance on the navigation tasks then become generalized to explain students' overall spatial abilities (e.g. Downs et al. 1988, Blades et al. 1995, Leinhaardt et al. 1998). 


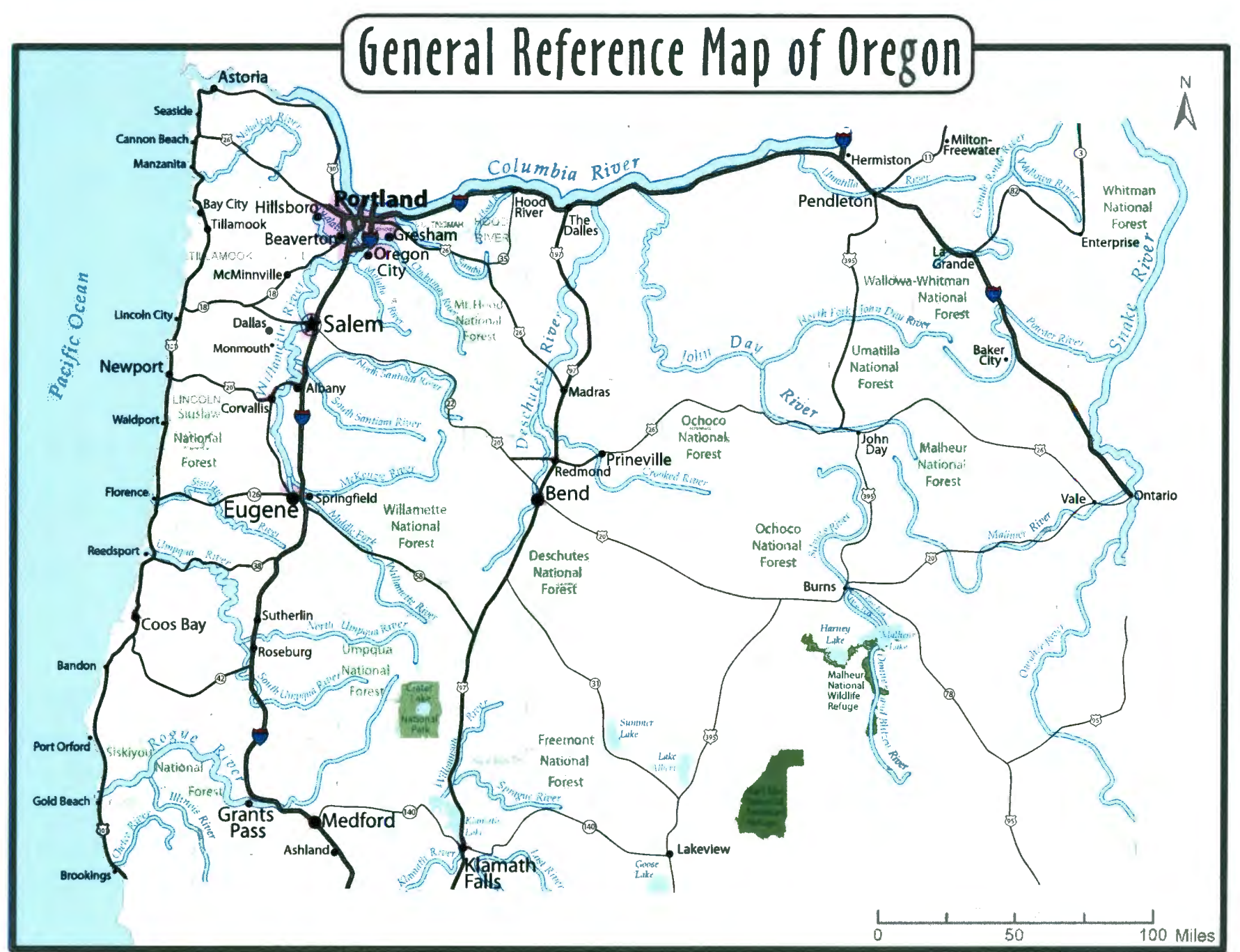

Figure 1. Example of a general reference map. All the features on this map exist as physical entitites on the ground where they are depicted on the map. 
Although research has mainly focused on general reference maps, most maps students encounter in school are thematic maps (Young 1994). Thematic maps are used to show spatial patterns of phenomena and communicate how they differ in both kind and amount (Slocum et al. 2005). Features on thematic maps may exist as physical entities at the symbol location, but frequently represent values derived from point samples or counts that are generalizations of broad patterns present in large areas as illustrated in Figure 2. Examples of information depicted on thematic maps include barometric pressure, median income levels, and population of towns. Because these maps depict generalizations of data to illustrate patterns of certain phenomena, they communicate a theme, hence the term "thematic."

Thematic maps can be further divided into three types based on the symbolization they employ: point, line, and area as illustrated in Figure 3 (Dent 1996). Point symbol thematic maps can show specific ground locations of phenomena or total quantities of data. Line symbol thematic maps show patterns of linear phenomena. Area symbol thematic maps can show either distributions of area phenomena or distributions of quantities in each area unit (Dent 1996).

Although there are numerous types of thematic maps available for use in the curriculum, researchers have found that few types are actually employed in the classroom, as illustrated in three particular studies discussed below. Not only are few types used, but those present in classrooms rely mainly on nominal level, or qualitative data. For the remainder of this thesis, "nominal scale data" may be 


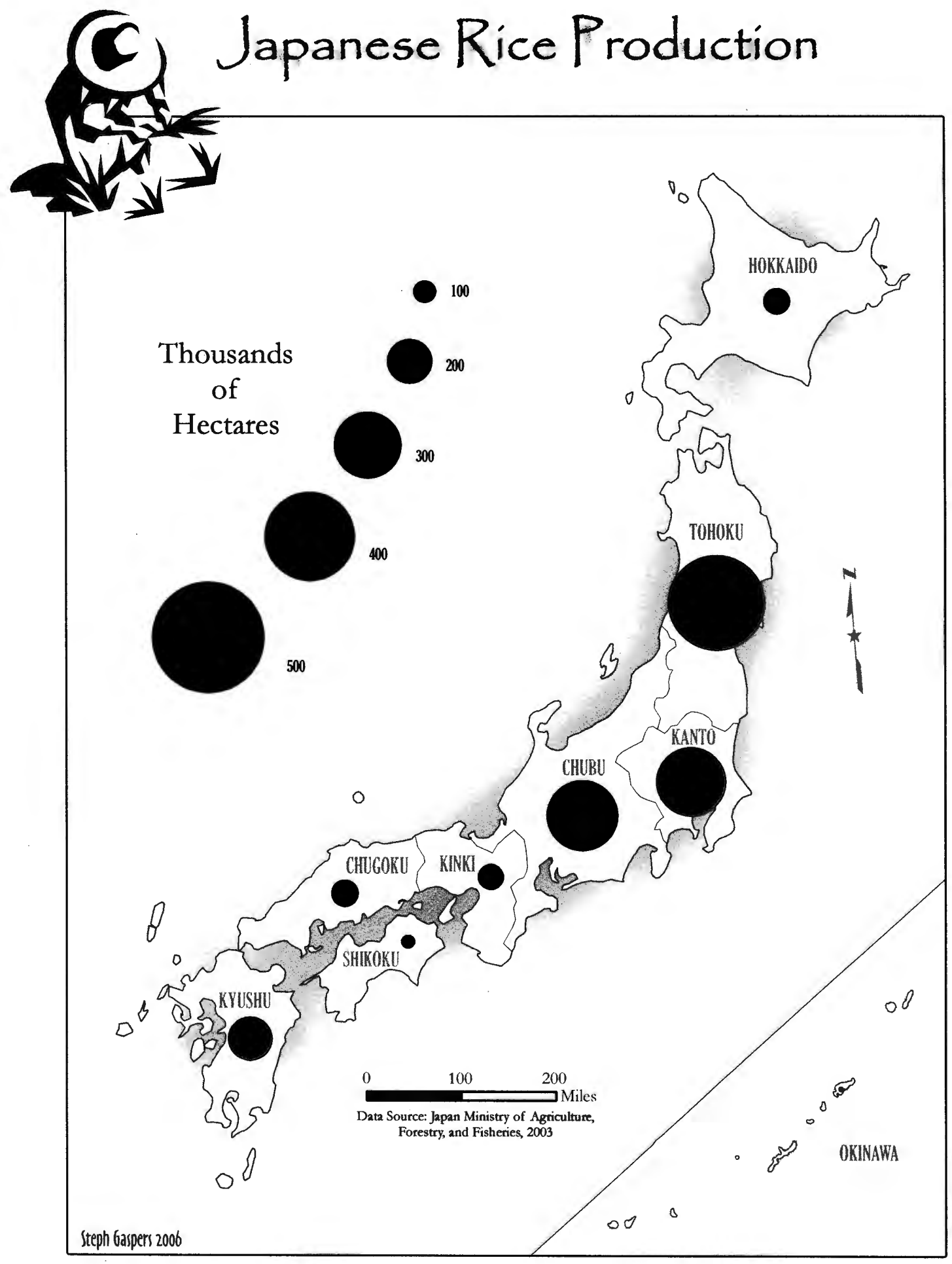

Figure 2. Example of a thematic map. It has different sized circles (the symbols) to represent hectares of rice produced by prefecture (the symbology). Its symbology was calculated to show generalizations of large areas, as the symbols cannot be found as physical entities on the ground as depicted. 

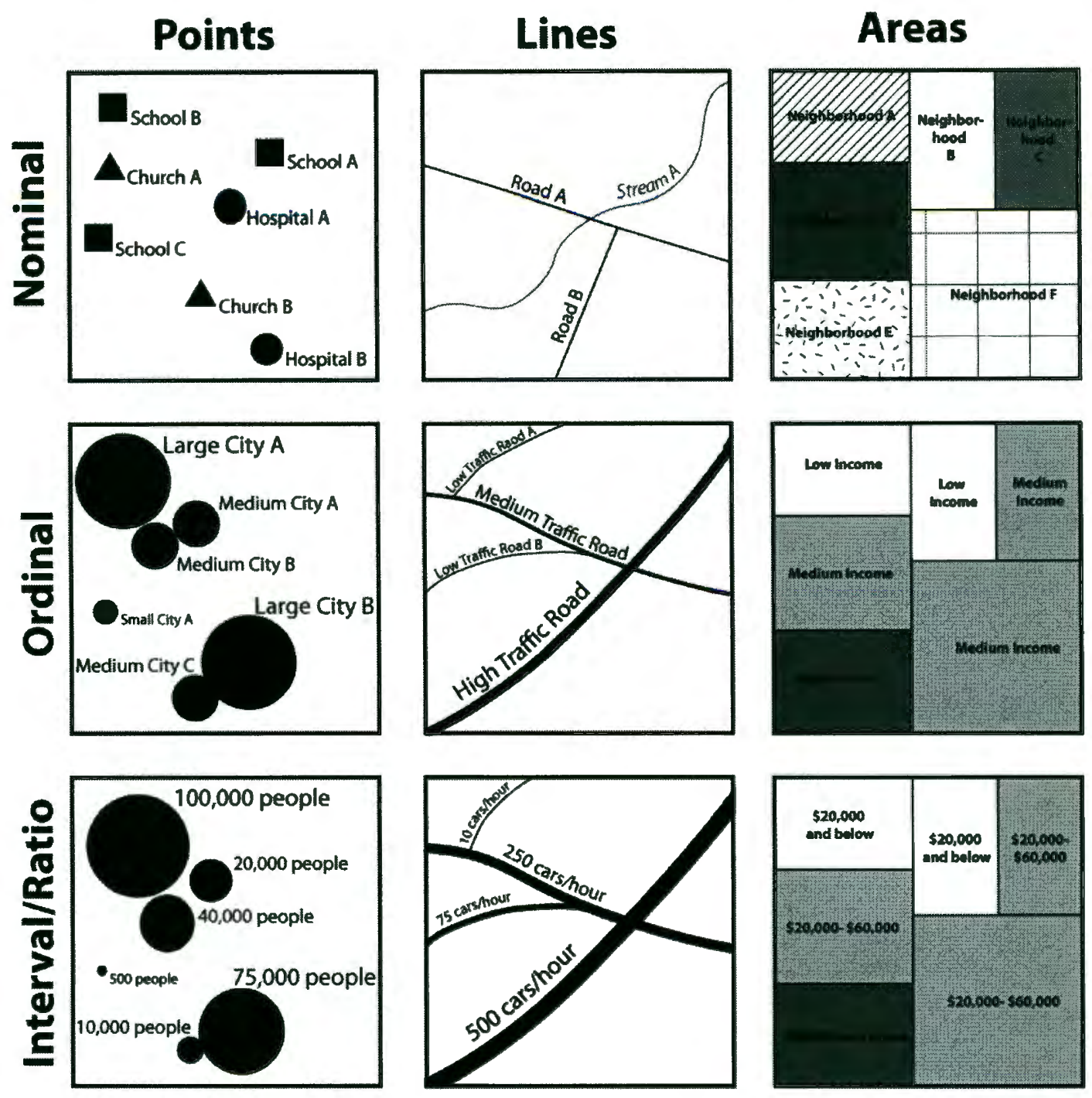

Figure 3. Thematic map symbol types. Point, line, and area symbols can display nominal, ordinal, and interval/ratio data with different symbolization. The interval/ratio data allows people to not only differentiate between kind, but also to compare quantities. Adapted from: O'Sullivan, D. and D. J. Unwin. 2003.

used interchangeably with "qualitative data" to refer to data that only represent

kind, and do not include information on amount. "Ratio scale data" may be used interchangeably with "quantitative data" to refer to data that identifies amount. 


\section{Review of Maps Used in Geography Curriculum}

Young (1994) examined twenty-six middle school social studies textbooks as part of his $\mathrm{PhD}$ dissertation and found that thematic maps are most frequently employed, compared to general reference maps, at a ratio of 3:1. Qualitative thematic maps in the form of "bounded area maps," also known as "color patch maps," comprised forty percent of these thematic maps. He notes that "textbook maps often depicted qualitative information and seldom showed quantitative data" (22). Young concluded that students can interpret quantitative symbols and suggested that more quantitative maps should be present in classroom instruction.

Castner (1997) also found a lack of a diverse representation of map types in school curriculum. He argues that children should be exposed to many different types of maps to expand their analytical skills, but notes that North Carolina curriculum only used three of the forty maps he identified as potential maps to use. He explains,

This suggests that we aren't doing a very good job of demonstrating to students the great diversity of map tools that are available to them and the tasks that are representative of geographic thinking, especially when data manipulation and mapping are involved. How will they, at the end of grade four, as Geography for Life asserts, be able to distinguish geographic from non-geographic questions? (59-60)

Castner argues that providing children with the opportunity to work with a variety of maps is necessary for them to reach the geographic goals educators deem important. 
I also conducted a survey of map types in twelve middle school social studies textbooks approved by the Oregon Department of Education (Appendix A). Of a total 1,258 color maps, 76 percent were thematic, while 24 percent were general reference. Similarly to Young's findings, I also discovered that over three quarters of the thematic maps depicted solely nominal level, or qualitative, data as summarized in Table 1.

Table 1. Results of middle school textbook survey. For more information on the types of maps presented in the table, see Dent (1996).

\begin{tabular}{|c|c|c|c|}
\hline Map Type & $\begin{array}{c}\text { Scale of } \\
\text { Measurement }\end{array}$ & $\begin{array}{l}\text { Map } \\
\text { Count }\end{array}$ & $\begin{array}{c}\% \text { of total } \\
\text { Thematic } \\
\text { Maps }\end{array}$ \\
\hline Color Patch & Nominal & 513 & 53.6 \\
\hline Point Symbol & Nominal & 132 & 13.8 \\
\hline Point \& Line Symbol & Nominal & 101 & 10.6 \\
\hline Flow Map & Nominal & 97 & 10.1 \\
\hline \multicolumn{2}{|c|}{ Nominal-Scale Sub-Total } & 843 & 88.1 \\
\hline Isopleth & Ratio & 90 & 9.4 \\
\hline Choropleth & Ratio & 11 & 1.2 \\
\hline Dot Map & Ratio & 6 & 0.6 \\
\hline Graduated Circle & Ratio & 5 & 0.5 \\
\hline Cartogram & Ratio & 2 & 0.2 \\
\hline \multicolumn{2}{|c|}{ Ratio-Scale Sub-Total } & 114 & 11.9 \\
\hline & Grand Total & 957 & 100.0 \\
\hline
\end{tabular}

Table 1 illustrates the strong reliance on map types that display only qualitative, or nominal scale data. It also illustrates that textbooks rarely include choropleth, dot, and graduated circle maps that are often used to present information to adults. If students have the ability to interpret quantitative map symbology, limiting the geography curriculum primarily to nominal scale data may underestimate students' abilities. 


\section{Research Objectives and Hypotheses}

To determine if choropleth maps, dot maps, and graduated circle maps are useful geographic tools for middle school students, I will test four hypothesis to identify whether students can effectively use these three quantitative thematic maps:

Hypothesis 1: Sixth-grade students can interpret symbolization used on graduated circle maps, dot maps, and choropleth maps.

Hypothesis 2: Sixth-grade students can make inferences by analyzing information on graduated circle maps, dot maps, and choropleth maps.

Hypothesis 3: Sixth-grade students can identify and classify regions of similar values on graduated circle maps, dot maps, and choropleth maps.

Hypothesis 4: Viewing symbologies on these maps can help students formulate geographic questions. 
I chose to test these four map reading skills because each provides different levels of understanding about the data on the map as illustrated in Figure 4.

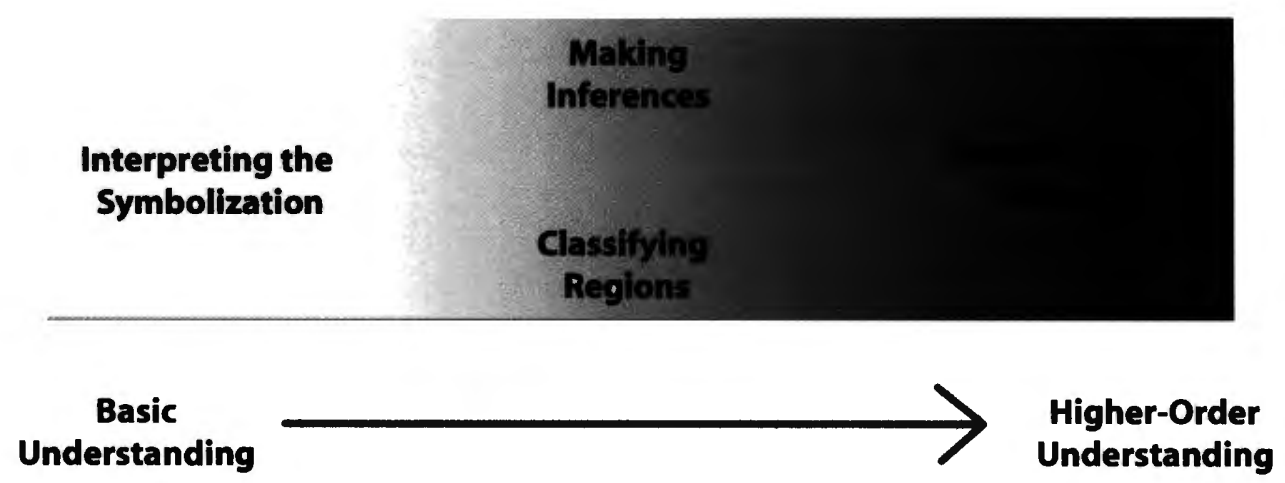

Figure 4. Levels of map reading skills.

Understanding symbology is the first step required before anything else can be understood about the map. Once values are comprehended, students must then be able to identify relationships between multiple attributes in one location or recognize relationships between many locations of one attribute so they can classify regions and make inferences (Robinson et al. 1984).

This should be a manageable task for middle school students. According to the Oregon Department of Education standards, at the end of fifth grade, students should have learned multiplication and division; rounding; algebraic operations involving representation of a mathematical variable (using a symbol such as a letter from the alphabet); and measurements of central tendencies (mean, median, mode, and range). Thus, middle school students do have experience with these mathematical skills that are required to understand 
quantitative thematic maps and they should be able to group similar values together into regions and/or make inferences about the data.

Once students fully understand the data by analyzing patterns, they can then begin to question why certain patterns exist and how the distributions are related to other phenomena's distributions. Breaking the broad term of "map reading" down into these four skills will also allow us to see if different maps are better for certain tasks instead of relying on an "overall" assessment that may generalize too much and give us a less accurate conclusion.

I chose to use the choropleth, dot, and graduated circle maps for several reasons. First, they rely on simple quantitative and graphical concepts that middle school students should be able to comprehend. Since the maps display quantitative data, they challenge students to recognize spatial patterns of amounts instead of simply requiring students to determine if one group is different than another as they would have to on qualitative maps. Second, these maps are the most underrepresented map types in textbooks. Third, these maps are widely used in atlases intended for adult audiences. Thus, they represent a level of knowledge that students will soon be attempting to reach. The results obtained from testing these hypotheses will allow us to understand sixth-grade students' ability to use these three quantitative thematic maps for analyzing patterns of spatial phenomenon. 


\section{CHAPTER 2:}

\section{LITERATURE REVIEW}

A combination of research from several disciplines is needed to provide a framework for my research questions. Studies on children's cognitive spatial abilities and cartographic design choices provide the contextual background to this thesis. A brief review of this literature is provided to explain these primary components.

\section{Spatial Cognition}

Geographers, psychologists, and education researchers agree that spatial cognition affects map reading ability (e.g. Downs et al. 1988, Rittschof and Kulhavy 1996, Rossano and Morrison 1996, Verdi and Kulhavy 2002, Blaut et al. 2003, Michaelidou et al. 2004). Human cognition is a complex mental activity that allows humans to perceive, learn, and reason about the world around them. Spatial cognition, or the process of acquiring knowledge about space, is just one component of human cognition, yet it is essential to human existence. Newcombe and Huttenlocher $(2000,1)$ explain that "spatial knowledge is essential to life in the world, since anything concretely existing in the world must have some spatial location."

Although spatial cognition is traditionally a subject that psychologists study, geographers are also interested in how people develop spatial understanding so that they can better communicate geographical concepts 
(Downs et al. 1988). This is especially true for those planning geography curriculum. They must know when certain concepts should be taught to students in order to correspond with a child's cognitive development. School is one source from which children learn skills important to adult life, including geographical inquiry. If geographers understand the theories psychologists have identified to explain spatial cognitive development, then better geography lessons can be created to advance learning.

Although spatial cognition includes many diverse aspects, this discussion will only focus on cognitive development that is pertinent to thematic map use. Cartographers often employ Piaget's cognitive development model because it details changes in children's spatial knowledge that can be tested in geographic research for which other psychological theories offer little explanation (Bluestein and Acredolo 1979, Downs et al. 1988, Downs and Liben 1991, Young 1994). Although Piaget's cognitive development theory provides an effective theoretical framework, cartographic researchers should also recognize that social structures, including school and family networks, may aid children in learning skills earlier than Piaget hypothesized, or conversely, retard that learning. In short, Piaget's work is a model and does not explain each individual case of development.

\section{Piaget's Theory of Cognitive Development}

Piaget's theory of cognitive development explains children's acquisition of hierarchical knowledge in stages. Piaget's theory is a linear model, where 
children cannot obtain knowledge in one stage before they have mastered knowledge in the previous stage as seen in Figure 5. Piaget explains that as

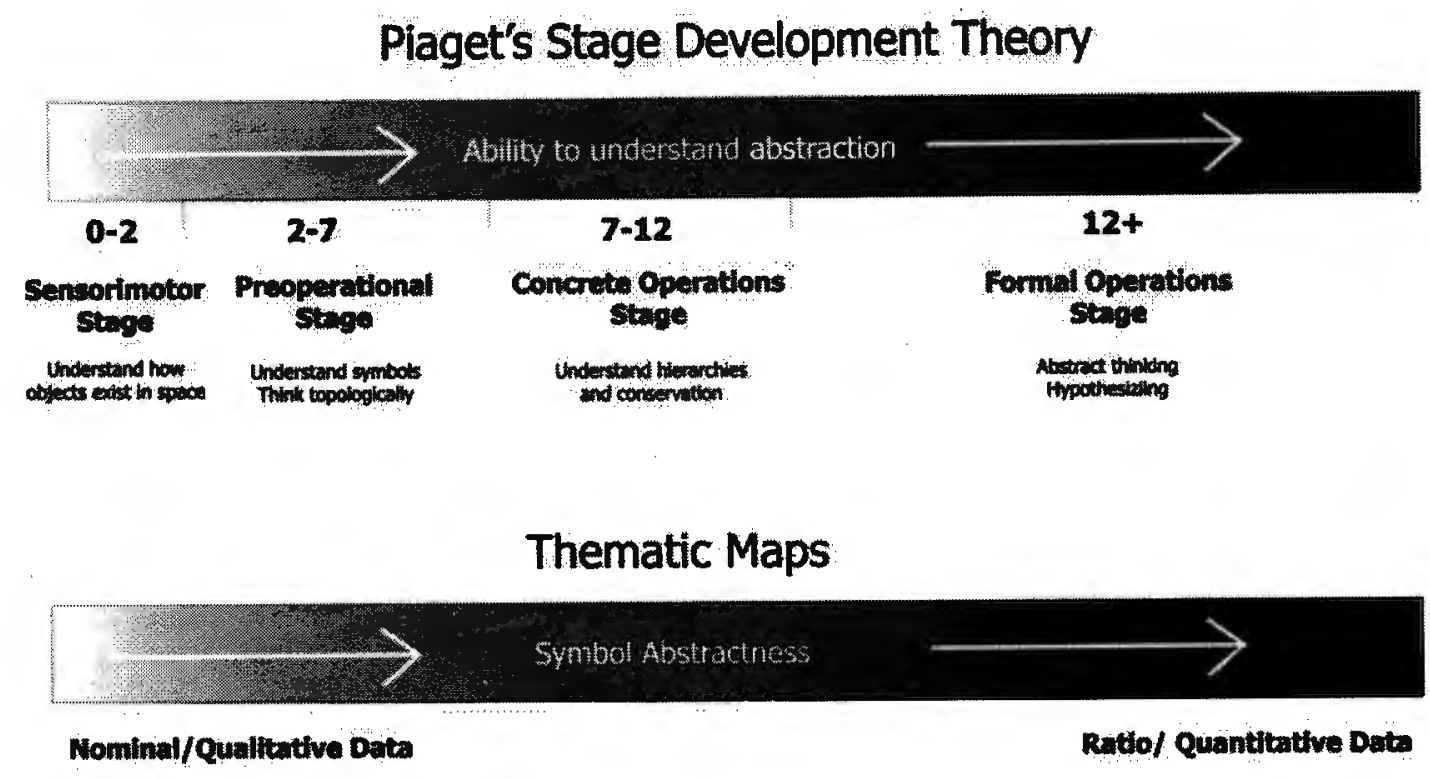

Figure 5. Approximate ages corresponding with Piaget's developmental stages. As children pass through development stages, they can understand more abstract concepts. This can be tied to the ability to interpret thematic maps. More abstract thinking is required to understand quantitative thematic maps than maps depicting nominal scale data.

children explore their world, they are confronted with unfamiliar information (Piaget 1970). They must either assimilate the new information into existing schema or create new schema to accommodate for information that will not fit into existing structures (Piaget 1970).

Piaget's first stage, the sensorimotor stage, is characterized by exploration. Children, beginning at birth, interact with objects in their environment and begin to understand how they exist in space (Hetherington et al. 2006). This is an 
essential building block for understanding spatial concepts necessary for geographic thought.

Once children understand objects' permanent positions in space, they move onto the second stage of cognitive development called the preoperational stage. Piaget asserted that children between the ages of two and seven developed essential spatial skills in this stage. They begin to understand symbols. In other words, they begin to understand that an object can stand for another object, an understanding essential to map reading. They also begin to categorize objects (Ault 1983) and understand objects' relationships as they develop topological thought processes (Newcombe and Huttenlocher 2000). This means that they can determine relative positions of objects. For example, they can understand when two objects are close to each other, inside each other, or distant from each other (Newcombe and Huttenlocher 2000). To identify patterns and relationships of spatial data present on maps, children must have developed this cognitive ability.

After they reach the preoperational stage of knowledge, at about the age of seven, they move into the stage Piaget terms concrete operations. Between the ages of seven and twelve, Piaget asserts children begin to understand more complex tasks. They begin to understand hierarchies and logical reasoning (Ault 1983). By the end of this stage, they will also master what Piaget referred to as conservation, or the idea that as objects change appearance, their basic properties remain constant (Hetherington et al. 2006). Figure 6 presents several examples of conservation. Conservation is important to map use because children must be 


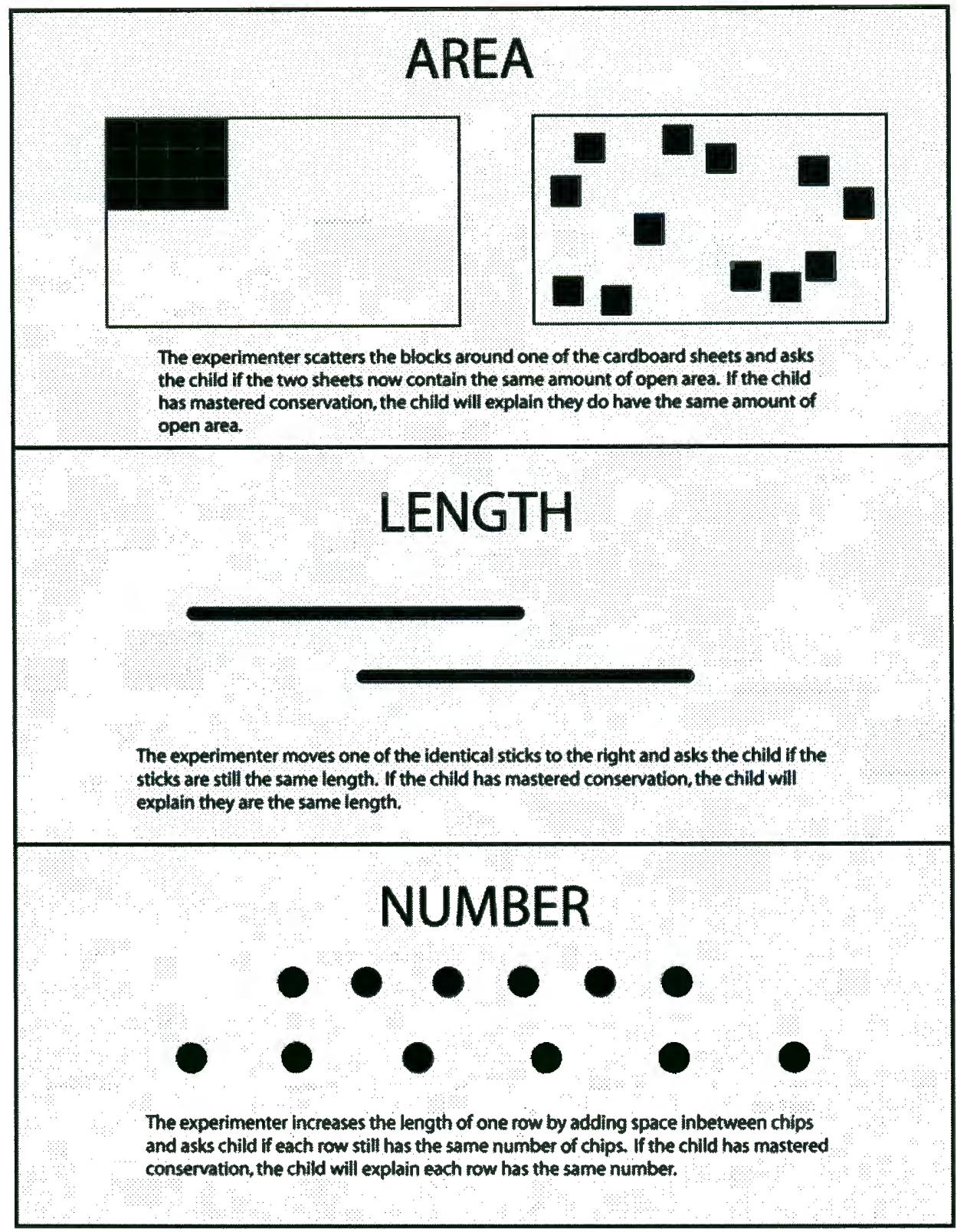

Figure 6. Examples of conservation problems. Once children master conservation, they understand that changing an object's position or arrangement in space does not change its physical properties such as area, length, or number. Adapted from Hetherington, E.M., Parke, R.D., Gauvain, M., Locke, V.O. 2006.

able to see that two values, such as county population densities, are the same regardless of shape or size of their respective enumeration unit before they can properly compare and contrast two regions on a map. 
After age twelve, children enter the formal operations stage where abstract thinking and hypothesizing occurs (Hetherington et al. 2006). Children begin to see spatial representations in projective and Euclidean space, meaning they can now picture abstract objects on arbitrary grids (Newcombe and Huttenlocher 2000).

\section{Vygotsky's Sociocultural Theory of Cognitive Development}

Piaget's identification of these developmental stages provides important guidelines for cartographers to consider when researching children's map skills. However, to help explain why some children develop certain cognitive skills earlier or later than Piaget would expect, Vygotsky's theory of cognitive development should supplement Piaget's theory.

Vygotsky reasoned that children's cognitive development is guided by interaction with people in society, including their teachers, peers, and parents. Of greatest interest to geographers is his concept of the zone of proximal development. Vygotsky argued that children have a range of possible achievement levels and that certain influences -- such as guidance from parents, teachers, or more experienced peers -- would cause children to obtain a higher understanding than if they tried to learn by themselves (Vygotsky 1978). This is often done through the process of scaffolding, where the parent or teacher gives less guidance to the child as he or she gains more understanding of a concept. When paired with Piaget's theory of cognitive development, Vygotsky's two concepts help explain why we 
should expect students to differ in abilities. Together, Piaget's and Vygotsky's theories provide at least a partial explanation of the mechanisms or processes that bring about cognitive growth and change that are essential to students' map reading abilities.

\section{Cognitive Processes Critical to Thematic Maps}

In order to understand the cartographic terminology I am about to use, I first must distinguish the differences between the word "symbol" and "symbology" as used in this thesis and illustrated in Figures 1 and 2 (on pages 5 and 7). I will use the word "symbol" to refer to the actual mark drawn on a map. It may be iconic, such as the traditional rail line symbol that closely resembles an actual railroad track, or it may also be abstract, such as a square or circle, used to represent towns. In Figure 2, for example, the "symbols" are the different sized black circles, or the actual marks on the paper. I will use the word "symbology" to refer to the meaning that the symbol carries. Therefore, in the same example, these black circles represent the amount of hectares devoted to rice production. I would argue that thematic maps employ more abstract symbologies because each symbol represents generalized information for an area on the map. It would seem reasonable that the cognitive skills required to read thematic maps differ from those needed for general reference maps because the symbols and symbologies thematic maps use are more abstract. 
To understand these more abstract thematic maps, children must be able to 1) interpret symbologies carried by each symbol, 2) compare and contrast regions, and 3) classify symbols and their representative values. Unfortunately, cartographers and psychologists have done little research on these types of cognitive abilities using experiments with maps (Young 1994). However, psychologists have explored children's abilities to perform these tasks in other situations that can be used to explain map reading.

\section{Symbology Understanding}

Carl Sauer, in his 1956 presidential address to the Association of American Geographers, observed that, "The map speaks across the barriers of language; it is sometimes claimed as the language of geography" (Sauer 1956, 289). Sauer's view of the role of maps provides a powerful introduction to symbol usage. In a sense, symbols on maps are like words in a book. Both are representations of real world phenomena. However, unlike language, symbols on maps are tied to geographic locations. They are symbols that not only represent phenomena, but also their place in space in relation to other objects, which may be more complex for children to understand (Newcombe and Huttenlocher 2000).

The cognitive structures needed to understand symbols appear in the preoperational stage when children begin to understand that language, models, and maps stand for real phenomena. As children experiment with basic symbols, they can begin to transfer the knowledge they gained about these earlier, more 
simplistic symbols to use in new, more abstract symbol situations (Marzolf and DeLoache 1994).

DeLoache, Miller, and Pierroustakos (1998) found that the more similar a pictorial symbol is to its real life object, the better children, especially between the ages of two and four, can understand its symbolization. Dent $(1996,16)$ refers to these pictorial symbols as replicative symbols and explains they represent tangible objects. Conversely, he refers to geometric shapes used to symbolize amounts as abstract symbols because they can "represent anything and require sophistication of the map user." For example, a replicative symbol imitating a house with windows and a peaked roof would be better recognized as a symbol for a house than would a dot placed in the same location. Thematic maps often employ abstract symbols and they may be too conceptual for preoperational children to comprehend. However, as discussed previously and illustrated in Figure 5 (page 16), as children advance through developmental stages, they begin to understand more abstract concepts such as maps that employ more abstract symbols.

For this reason, thematic maps are introduced in schools to children in formal and concrete operations stages. Marzolf and DeLoache (1994) conclude that children understand more abstract symbols better if they are presented with more concrete representations previously. Therefore, I would also argue that if children are presented with less abstract thematic maps at an earlier age, by the time they reach the formal and concrete operation stages, they should be ready to understand the next level of complexity added to the map: more abstract 
symbologies. These symbologies not only represent elements on earth, but also represent certain numerical values associated with the symbols. Thus, once children understand this concept, they obtain basic a understanding of the map.

\section{Ability to compare and contrast}

The next step of thematic map reading geographers are interested in is the ability to compare values between regions. Children must understand that thematic map symbols show quantitative values. They also must understand that symbols and their respective values can be compared to determine values that are larger or smaller than other symbols' values. In the preoperational stage, children have limitations on their abilities to understand what Piaget refers to as conservation. Thus, preoperational children have difficulties understanding that objects retain their area, length, and number despite their arrangement in space as explained in Figure 6. This can affect map reading ability greatly. For example, look at the choropleth map in Figure 7. The fact that County A and County C have the same population density may not be understood by children who have not mastered conservation because County $\mathrm{A}$ has a much larger area than County C.

Although research has not tested this, a preoperational child, not fully comprehending conservation, may argue that County A has a higher density because it has a larger area. Kotovsky and Gentner (1996) found this to be true in 

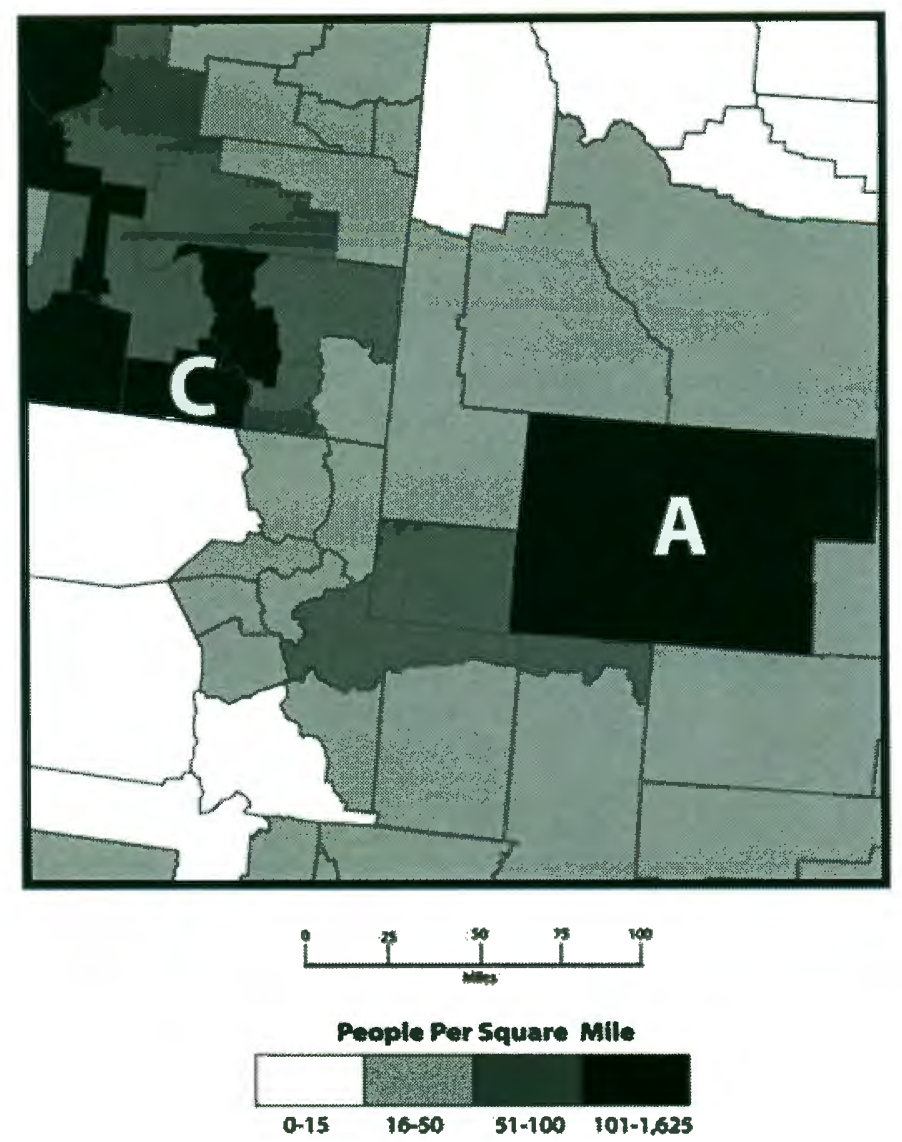

Figure 7. Choropleth map depicting population density.

an exercise comparable to interpreting map symbologies. They tested children's ability to distinguish between two shades of gray on different sizes of geometrical shapes. They found that four-year-olds could only compare objects' colors if they were the same shape and size, but six- and eight-year olds were better at comparing objects' colors regardless of shape or size, although some were better at it than others.

Although this may be a problem that children with less-developed spatial cognitive abilities have, according to Piaget, by the time children reach middle 
school when they are aged eleven or twelve years, they should have mastered conservation knowledge and be able to correctly compare and contrast values.

\section{Categorization}

Third, geographers are interested in the spatial cognitive processes required to categorize thematic map symbologies. Categorization "reduces the diversity of the physical world" (Quinn 2002, 85). The purpose of thematic maps is to show spatial distributions of phenomena in order to allow readers to identify patterns or relationships. For example, in order to comprehend the graduated circle map seen in Figure 8, a person must categorize the circles somehow to make sense of the map.

One approach might be to assign all the larger circles in the northern part of the map into a group and categorize all the smaller circles in the southern portion of the map into another group. Once this is done, one can begin to ask, "why are there more people in the northern part of the state than in the southern portion?" Therefore, categorization is a critical underpinning for geographical inquiry.

The most common experiment that psychologists employ to test these abilities is to evaluate if young children can categorize figures such as play toys into different groups. For example, Mandler, Bauer, and McDonough (1991) found that younger children between seven and twenty-four months can separate global representations such as dogs from trucks into groups, but had difficulty 

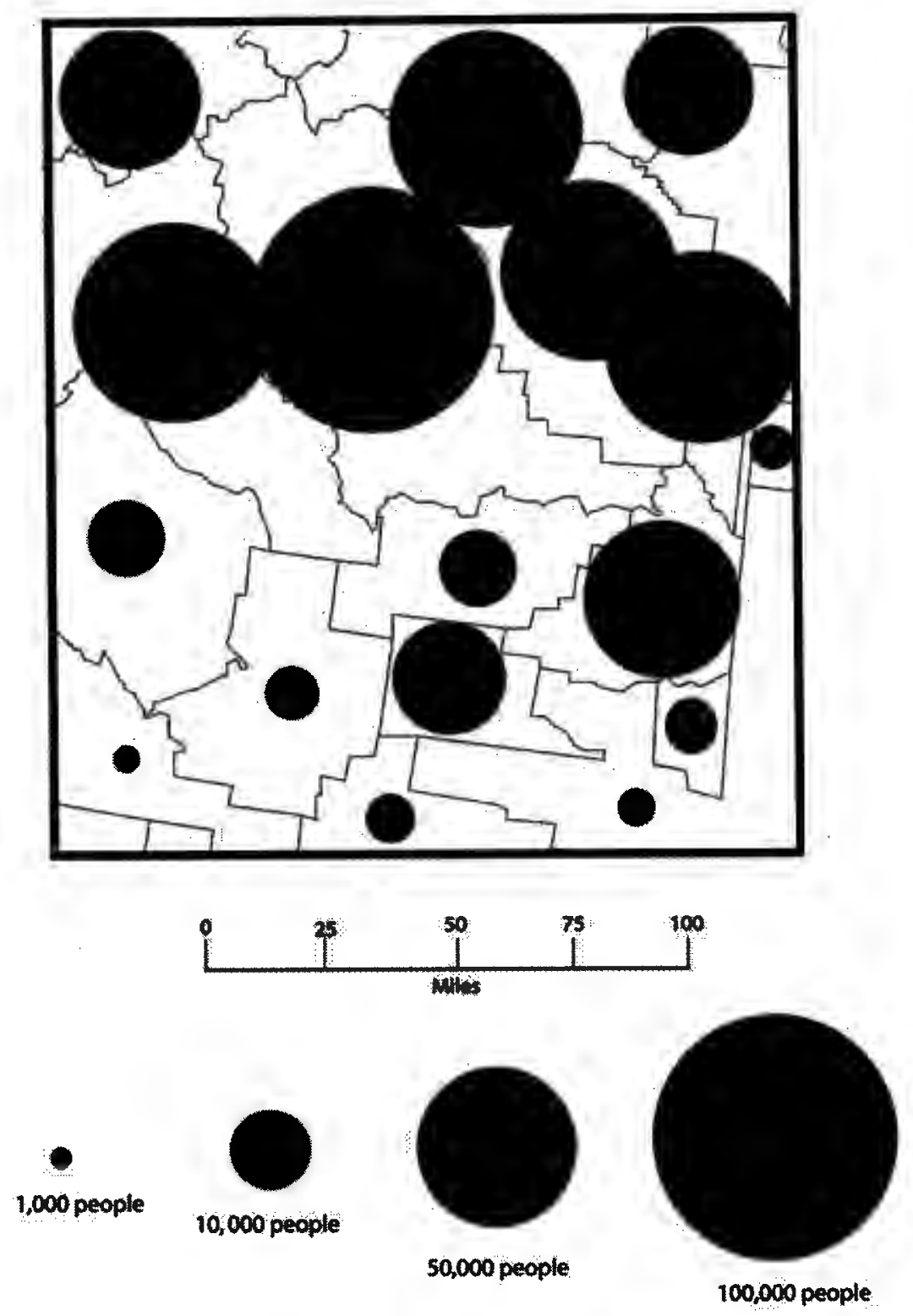

Figure 8. Graduated circle map depicting county population.

grouping dogs into separate groups than cats. This suggests that younger children do have the ability to group, but it is not thoroughly developed.

Another study that may be more applicable to map reading tested children between the ages of four and eight on their ability to group different colored 
symbols drawn on a sheet of paper according to proximity and similarity (LangeKüttner 2006). The results indicate that coding common regions according to similarity and proximity emerged at age five and improved with age. LangeKüttner discusses that children between four and five focus on each symbol individually and as a result have difficulty recognizing overall patterns. However, by age eight, most children were able to see regional patterns because they first create a plan by assessing the overall image. Lange-Küttner concluded that younger children do not scan the entire picture and then create a plan to categorize, but instead begin to group based on the first symbol they encounter. This may indicate that younger children are consumed with the task of interpreting symbologies and therefore have difficulties grouping similar, nearby symbols; whereas older children interpret symbologies and are able to focus on defining rules that govern what is considered a "group." These findings and suggestions can be directly applied to how children understand map symbologies.

\section{Cognitive abilities directly tied to cartography}

The studies conducted within the field of psychology offer some explanation of children's cognitive abilities that can be applied to thematic map reading, but cartographic research on children's abilities to understand thematic maps is less frequently published. Two $\mathrm{PhD}$ dissertations examined children's ability to understand thematic maps. Young (1994) tested 335 children from ages eight to fourteen years to record their performances on dot, graduated circle, 
isoline, choropleth, and color patch maps. He found that younger children had wider ranges of scores than did older children, but all children showed some ability to understand thematic maps. Young also found that differences existed between children's ability to understand different symbologies.

He asked the children to answer five multiple choice questions about each map. This required them to compare quantities for ratio scale data maps or qualities for nominal scale data maps. For example, on the choropleth, dot, and graduated circle maps, children were asked, "Which county has the most people?" or "How many people live in county D?" or "Which county has a large city with many people?" Each question was then followed by four choices from which the student could circle their answer. The mean scores for each map are included in Table 2.

Table 2. Young's Results of Students' Map Reading. Mean scores (out of a possible 5) of 8-14 year olds' performance on each map type illustrate that students performed least well on line symbol maps (isoline) and best on area symbol maps (choropleth and bounded area).

\begin{tabular}{rc} 
Symbolization & $\begin{array}{c}\text { Mean } \\
\text { Score }\end{array}$ \\
\hline Isoline & 3.2 \\
\hline Graduated Circle & 3.8 \\
Dot & 3.9 \\
\hline Color Patch & 4.6 \\
Choropleth & 4.7 \\
\hline
\end{tabular}

Young found students' performance on each of the map types falls into three statistically significantly different groups. First, the children scored highest 
on choropleth and bounded area maps. They scored second highest on point symbol maps that include dot and graduated circle maps. They performed least well on the line symbol map. He found there was no difference between sexes and concluded that "children develop some of the cognitive abilities necessary to understand thematic maps at a younger age than Piagetian theory would predict" (Young 1994, i).

Similarly, Trifonoff (1994) studied 74 second-grade students and found that they were able to recognize spatial patterns on both choropleth and graduated circle maps. Additionally, she found that second-grade students were able to interpret the abstract symbologies used on these maps by answering if one county had a higher or lower value than another. These studies contribute to the underresearched area of thematic mapping abilities of children and affirm that children do possess spatial cognitive skills to understand thematic maps.

Piaget's theory provides important contributions to understanding how children gain knowledge of spatial concepts. However, as noted by Blaut et al. (2003) cognitive development does not occur by act of pure genetics and biological processes. Children are affected by social interaction, such as guidance from parents or teachers. This is important to know so that Piaget's theory provides an underlying framework, not an age-specific manual, that cartographers and geographers can work from. Because primary map skills such as orientation and direction are taught early in school, children obtain basic geographic concepts that can be added to as they encounter more complex concepts. Because there are 
geography standards present in all public schools requiring early use of maps, students should be ready to be exposed to complex thematic maps in middle school to add more abstract geographical understanding to their cognitive structures. 


\section{CHAPTER 3:}

\section{METHODOLOGY}

\section{Map Design}

Cartographic research has established general guidelines for those interested in effective design choices for map elements specific to each map type. These design choices include decisions on colors, symbols, categorization, generalization, and typeface. They help cartographers design clear and concise maps that provide the best opportunity for map readers to understand the presented information. Cartographic research has also concluded that differences in data handling and representation produce distinct symbolization for each map that requires different visual tasks by the reader. The distinctive symbolizations result in separate strengths and weaknesses of each map type that may affect students' abilities to read, comprehend, and analyze the data. These design principles and map characteristics are formally explained in Appendix B and outlined in Table 3. Additionally, the few who do offer guidelines for cartography specifically aimed at children have been incorporated into the design of the maps (Gerber 1984, Wiegand 2003), however the suggestions these researchers offer do not drastically differ from findings discovered by cartographers specializing in thematic maps for adults.

For this study, I designed three thematic maps displaying Oregon population: a choropleth map, a dot map, and a graduated circle map. Each map 
Table 3. Comparison of thematic maps. Found in Figures B1, B2, and B3 in Appendix B.

\begin{tabular}{|c|c|c|c|}
\hline & $\begin{array}{c}\text { Dot Map } \\
\text { (App, B, Figure B1) }\end{array}$ & $\begin{array}{l}\text { Graduated Circle Map } \\
\qquad \begin{array}{c}(A, p p, B, \text { Figure } B 2) \\
\end{array}\end{array}$ & $\begin{array}{l}\text { Choropleth Map } \\
\text { (App-B, Figure B3) }\end{array}$ \\
\hline $\begin{array}{l}\frac{5}{0} \\
\frac{0}{3} \\
\frac{N}{0} \\
\frac{0}{E} \\
\text { के }\end{array}$ & $\begin{array}{l}\text { - Uses one dot to } \\
\text { represent a specified } \\
\text { count of data. } \\
\text { Dot value and dot size } \\
\text { decisions combined } \\
\text { with dot placement } \\
\text { produces a pattern of } \\
\text { dot densities. } \\
\end{array}$ & $\begin{array}{l}\text { Uses different-sized } \\
\text { circles to represent } \\
\text { different counts of data. } \\
\text { - One circle is placed for } \\
\text { each enumeration unit. }\end{array}$ & $\begin{array}{l}\text { - Uses shades of hues to } \\
\text { represent high and low } \\
\text { densities of phenomena } \\
\text { by a chosen enumeration } \\
\text { unit. } \\
\text { - An entire enumeration } \\
\text { unit is shaded to represent } \\
\text { the density class it is in. }\end{array}$ \\
\hline$\frac{\text { 을 }}{\frac{5}{5}}$ & $\begin{array}{l}\text { Raw data counts are } \\
\text { mapped. } \\
\text { Total data count } \\
\text { divided by dot value } \\
\text { equals total number of } \\
\text { dots. } \\
\end{array}$ & $\begin{array}{l}\text { - Raw data are mapped. } \\
\text { - Data values are } \\
\text { mathematically scaled to } \\
\text { equal the area of the } \\
\text { circle. }\end{array}$ & $\begin{array}{l}\text { - Densities or ratios are } \\
\text { mapped. } \\
\text { - Data grouped into classes } \\
\text { or ranges of values. }\end{array}$ \\
\hline $\begin{array}{l}\frac{c}{0} \\
\frac{0}{0} \\
\frac{2}{2} \\
\frac{1}{2} \\
\frac{0}{0} \\
0\end{array}$ & $\begin{array}{l}\text { Ancillary data are } \\
\text { used to determine } \\
\text { where phenomena } \\
\text { most likely occur. } \\
\text { Dots are placed in } \\
\text { local areas or zones of } \\
\text { homogeneity. }\end{array}$ & $\begin{array}{l}\text { At small/medium } \\
\text { scales, circles are placed } \\
\text { at points such as cities. } \\
\text { - At medium/large scales } \\
\text { circles are placed at } \\
\text { centroids of polygonal } \\
\text { enumeration units }\end{array}$ & $\begin{array}{l}\text { - Shading is applied } \\
\text { uniformly over the entire } \\
\text { enumeration unit. } \\
\text { Dark shades equal high } \\
\text { densities, light shades } \\
\text { equal low densities. }\end{array}$ \\
\hline 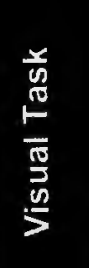 & $\begin{array}{l}\text { Determine relative } \\
\text { densities of dots. } \\
\text { The higher the density } \\
\text { of dots, the more data } \\
\text { present in that area. }\end{array}$ & $\begin{array}{l}\text { - Estimate size of circles. } \\
\text { - The larger the circle } \\
\text { size, the more data are } \\
\text { present in that area. }\end{array}$ & $\begin{array}{l}\text { Organize tones into spatial } \\
\text { regions of similar values. } \\
\text { The more similar tones are } \\
\text { to neighboring tones, the } \\
\text { more homogeneous the } \\
\text { phenomena. }\end{array}$ \\
\hline 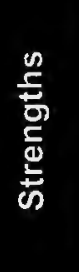 & $\begin{array}{l}\text { Allows readers to see } \\
\text { patterns (Jenks 1974, } \\
\text { Dent 1996). } \\
\text { Provides for a wide } \\
\text { range of densities to be } \\
\text { portrayed. }\end{array}$ & $\begin{array}{l}\text { - Allows readers to } \\
\text { quickly identify and } \\
\text { compare regions. } \\
\text { - Simplifies values for } \\
\text { complex area outlines to } \\
\text { a set of discrete points. }\end{array}$ & $\begin{array}{l}\text { Allows readers to quickly } \\
\text { compare regions. } \\
\text { Tonal values are } \\
\text { intuitively assigned to } \\
\text { high-low numeric values. }\end{array}$ \\
\hline 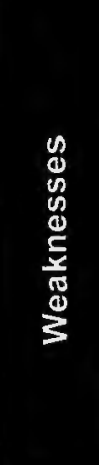 & $\begin{array}{l}\text { Variations of high and } \\
\text { low dot densities are } \\
\text { not classified similarly } \\
\text { by all people (Jenks } \\
\text { 1974, Provin 1977, } \\
\text { Mashoka et. al 1986, } \\
\text { Sadahiro 2000). } \\
\text { Actual data count } \\
\text { numbers are difficult } \\
\text { to determine (Provin } \\
\text { 1977, Sadahiro 2000). }\end{array}$ & $\begin{array}{l}\text { - Perception of circle size } \\
\text { can be affected by other } \\
\text { factors that result in } \\
\text { miscalculation of data } \\
\text { amounts (Flannery } \\
\text { 1971, Gilmartin 1981). } \\
\text { - Circles for polygons may } \\
\text { mask variation within } \\
\text { the polygon. }\end{array}$ & $\begin{array}{l}\text { - Masks density differences } \\
\text { within enumeration units. } \\
\text { - Classification process may } \\
\text { result in misleading } \\
\text { groups } \\
\text { - Continuous symbol for } \\
\text { discrete data may be } \\
\text { misleading. } \\
\text { - Variable enumeration unit } \\
\text { size may impact } \\
\text { interpretation. }\end{array}$ \\
\hline
\end{tabular}


is approximately 7" $\times 4$ " and is printed in color in portrait format on $81 \frac{1}{2}$ " $\times 11$ " white paper. All three maps are derived from the same Oregon 2000 population data obtained from the United States Census Bureau (2000). However, each map uses its distinctive symbolization to show the data.

The choropleth map has a sequence of five orange tones and data are categorized using Jenks Optimal classification in ArcGIS 9.2. The dot map uses black dots on pale yellow with a scale of one dot equal to 500 people, or roughly one third of the lowest population of the thirty-six counties in Oregon. The graduated circle map uses solid black graduated circles directly scaled to the data values. Because I am not testing the effects of color on students' abilities to analyze maps, color was introduced into these maps only in order to closely resemble maps found in textbooks. The orange color palette is often used to show population. When dot maps and graduated circle maps are used, they usually display black dots/circles on a pastel base map.

Although the maps created for this study reflect a number of subjective decisions, they do uphold cartographic design guidelines widely recommended by the literature. Also, they closely resemble maps found in middle school textbooks, suggesting they are similar to maps students would encounter and be expected to use. 


\section{Questionnaire Design}

Nine questions accompanied each map type. The complete questionnaire packet for each map type can be seen in Appendix C. All but one of the nine questions are identical for each of the three maps in order to allow direct comparison of students' performance between map types. The one question that is unique to each map should determine if students understand their specific map's symbolization, and thus must be asked differently to accommodate each of the three symbolizations.

Five questions were asked to evaluate students' abilities to interpret, infer, and inquire about the data on the map. Three questions generated personal information to characterize the subjects by age, gender, and their opinion of the test questions. These questions were used to test differences between distinguishable human traits that may affect map reading ability, but are not as important to the study as the actual test questions about the map content.

These questions were combined into a four-page packet, each packet presenting just one map type. The first three pages each have the same map at the top of the page with one or two questions about the map below. The purpose of reproducing the map three times is to prevent confusion resulting from students flipping between pages to refer back to one map. Additionally, it allows students to focus on the question, not where to find the information, thus resulting in more accurate answers. Lastly, this format separates the students' 
responses into a less crowded area that makes it easier for the students to record their answers and for the tester to read and interpret their responses.

Questions 1 and 3: Interpreting the Symbolization

1. On the map at the right there is a highlighted county. Find the same county in the map above. According to the map above, how many people would you estimate live in that county?

3. On the map at the right, look at the highlighted County $A$ and County B. Find these counties in the map above. Looking at the map above, which county has more people: county $A$ or county $B$ ?

Questions 1 and 3 are designed to analyze students' ability to understand the map and its symbolization. They are not included consecutively and on the same page in the survey because of formatting and logistical purposes. Young (1994) asked similar questions, but in a multiple choice format. I have changed the answers to short answer to reduce the likelihood of obtaining the correct answer by chance. Additionally, regardless of whether it is multiple choice or not, students must consult the legend and determine county population size before answering the questions.

Question 2: Making Inferences

2. Mark an $X$ in one county on the map at the top of the page that you think has a city with many people living in it. There may be more than one correct answer, but only put an $X$ in one county. 
Question 2 requires students to make inferences based on the data, an essential task to comprehending the map. Young (1994) also required students to make inferences with similar questions, but again asked the questions in a multiple choice format. Because I am not asking multiple choice questions for reasons noted above, I have explained in the question that "there is more than one correct answer" to help minimize confusion for students.

\section{Question 4: Spatial Pattern Recognition}

4. Geographers say that a region is an area that includes more than one county. A region does not have to follow county borders. Many people see regions of high, medium, and low population on the map at the top of the page. On the map at the top of the page, draw lines around the regions that you think are high, medium, and low and label each with the word "High," "Medium," or "Low." When you get done, every place in Oregon should be in a high, medium, or low region.

Question 4 requires students to think about spatial patterns. This requires two skills: the ability to interpret symbols and the ability to categorize similar values. Comparable tests were performed by Jenks (1974) where he was able to determine where college students saw high and low density patterns on dot maps. His study provides evidence that people can classify regions on maps and there are "normal" or "usual" patterns that emerge. He then researched which individuals deviated from the "normal" patterns. I am not interested in which individuals deviate from the expected, but rather interested in identifying: a) whether children can also recognize spatial patterns to classify them into regions; 
and, b) whether there are any patterns of region drawing performance either

within or between map types. The final sentence of the question is intended to force students not to simply identify the "easy" areas of high and low, but to address more difficult issues of categorizing all data.

\section{Question 5: Spatial Relationships and Inquiry}

5. Imagine that you are a reporter from another state and you wanted to write a story about where people live in Oregon. You look at this map as a starting point and learm where people live in Oregon. However, looking at the map and where it shows people living, you begin to think of other questions about Oregon's population. If you can find answers to these questions, it will help you write an informative story on Oregon population. Before you do any more research, you need to have questions that you will try to find answers to. So, think of some questions you have about Oregon's population by looking at the map.

In the space below, write down 2 questions that the map makes you ask about where people live in Oregon that will be important to writing your story. Do not write the answers, just the questions.

Question 5 requires students to examine spatial relationships and record the inquiry process it evokes. Requiring students to identify a question is a technique similar to a technique Gregg (1997) used. She determined that students who posed questions about maps understood the map more thoroughly than those who did not. However, the way she phrased her questions required students to ask questions about map design. Thus, the answers she obtained reflected how well students understood the map's elements and navigational properties. I want to determine if students can understand the data and their 
distribution patterns and what those patterns mean for other geographical phenomena associated with population. For example, students should recognize that there are parts of Oregon that are more populated than others (and are tested on this ability in Question 4). Geographic questions probing the cause or ramification of these distributions, such as "Why do few people live in Eastern Oregon and many people live in Western Oregon?" should be the next step toward a fuller understanding of the geographic data. The answers to this question will determine: a) whether students do develop questions by looking at thematic maps as the National Geography Standards would like them to; and b) whether there are differences between the types of questions resulting from each map.

\section{Question 6: Symbolization Understanding}

Question 6 is different for all three maps because it requires students to explain the symbology of their specific map, showing they fully understand how it "works." Question 6 for each of the three maps are as follows:

Choropleth:

The whole map is shaded a color, but people don't live in every square mile in Oregon because there are forests, lakes, and rivers where people cannot live. But, the map is still correct. Explain how:

Dot

People live in parts of Oregon where there are no dots on the map, but the map is still correct. How can this be?

Graduated Circle

People live in areas in Oregon that aren't covered by the circle in each county, but the map is still correct. How can this be? 
The purpose of this question is to provide more insight on students' performance about the survey and uses language more appropriate for sixth-graders than directly asking them to "explain how the map works."

\section{Sample}

After obtaining approval from the human subjects review board

(Appendix D), I identified eight sixth-grade classes in which to conduct this study as presented in Table 4. Two hundred and two students, both male and female aged 10-13 (Figure 9 and Figure 10) participated in this study. Each student participated voluntarily.

Table 4. Participating sixth-grade classes.

\begin{tabular}{ccc} 
Location & Teacher & $\begin{array}{c}\text { Number of } \\
\text { Students }\end{array}$ \\
\hline \hline Fairview, OR & A & 23 \\
Eugene, OR & B & 24 \\
Eugene, OR & B & 30 \\
Eugene, OR & B & 24 \\
Beaverton, OR & C & 27 \\
Beaverton, OR & C & 23 \\
Beaverton, OR & D & 26 \\
Beaverton, OR & D & 24 \\
\hline & Total & 202
\end{tabular}




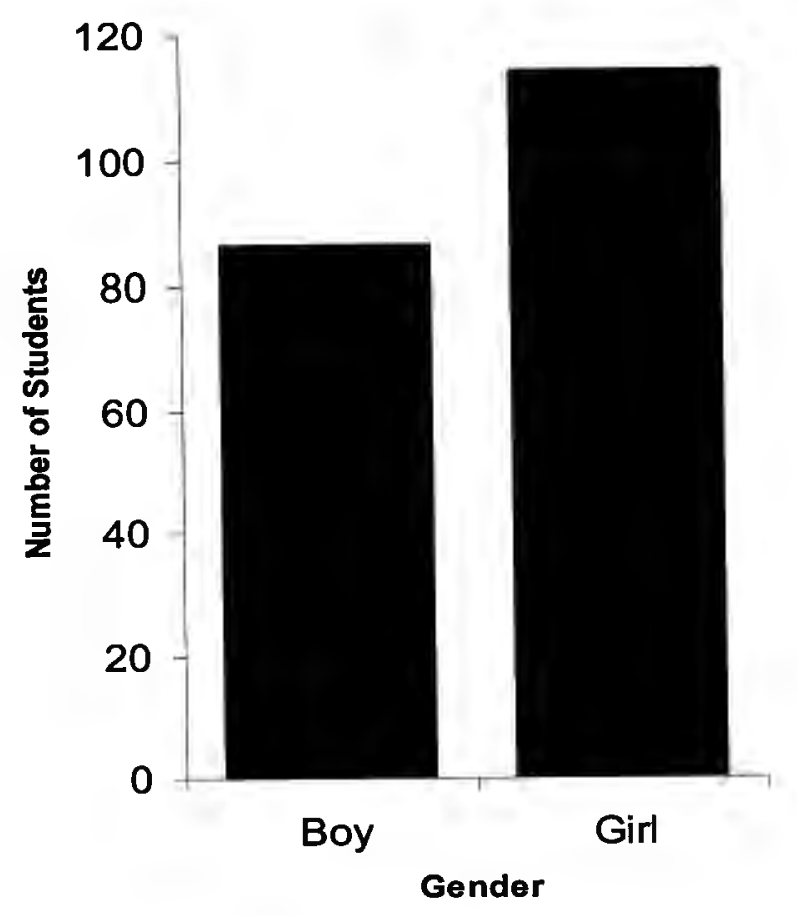

Figure 9. Number of each gender that participated.

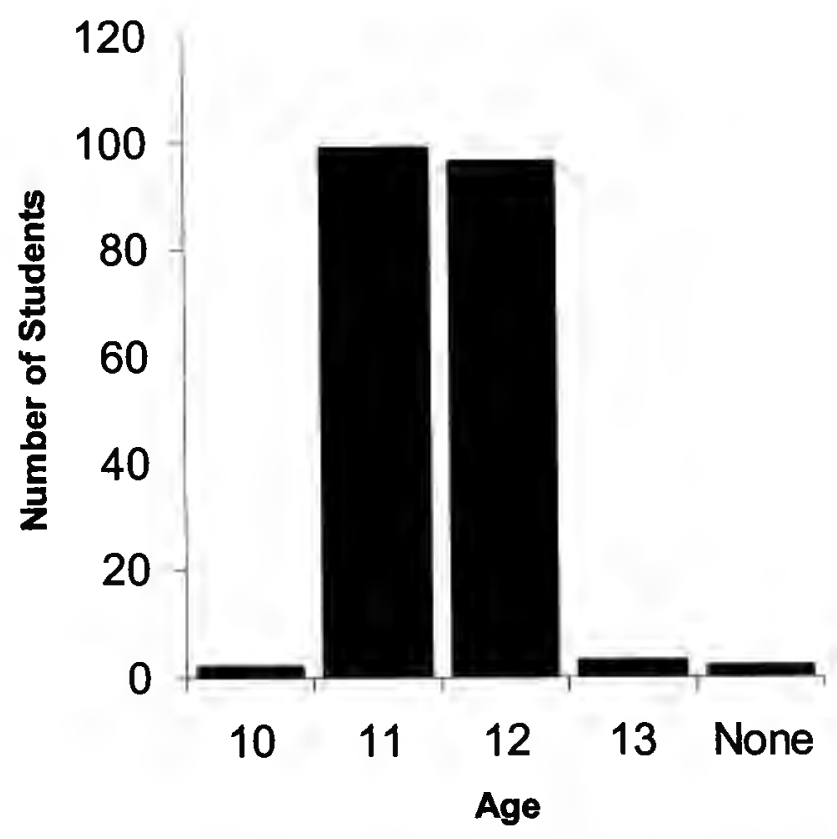

Figure 10. Age of participants. Most students included in the study were 11 and 12 years old. Two students gave no age. 


\section{Data Collection}

A helper and I went to the eight sixth-grade classrooms between February $21^{\text {st }}$ and February $28^{\text {th }}, 2007$. All surveys were distributed in the social studies portion of the students' class time. In Oregon, all sixth-graders are enrolled in a social studies class that includes lessons on geography. The first class we visited was in Fairview, OR. It served as a pre-test to determine if the questions could be realistically answered by students. Because this pre-test did not identify any crucial problems, I kept the format of the questions and included this class' responses in the study.

Within each class, my helper distributed each of the three map type packets randomly to students, so approximately one third of the class analyzed the dot map, one third analyzed the graduated circle map, and one third analyzed the choropleth map. As the maps were being distributed, I read the instructions from a script (Appendix E) that directed students to keep their map packet face down on their desks until all the instructions were read. I emphasized that any time during the exercise if the students had questions, they could raise their hand and I or my helper would assist them. Once the instructions were clear, the students were directed to turn over their map packet and answer the questions at their own pace. The students marked answers directly on their maps/answer sheet. When they finished, they were instructed to raise their hand so we could collect their packet and record the time it took them to finish the exercise. A total 
of 202 map packets were collected: 68 choropleth maps, 69 dot maps, and 65 graduated circle maps.

\section{Data Analysis}

I entered the data we collected from the students into an Excel spreadsheet for tabulation and summarization. I assigned a number to the test packet that links it back to the teacher and class, but retains students' anonymity because no names were collected. I then analyzed these data in SPSS 12.0 using Pearson's Chi-Square test to measure goodness of fit. The chi-square index measures whether the observed frequencies of correct and incorrect answers deviate from frequency distributions expected by chance (McGrew and Monroe 2000). If the frequencies deviate from expected frequencies beyond a $95 \%$ confidence interval, the chi-square test will produce a statistically significant result. A statistically significant result indicates that correct and incorrect answers for one or more of the map types deviated greater than could be expected by chance. In other words, one or more of the map types presented data more or less effectively than expected for the task.

To determine which map contributed to the significant chi-square result, I consulted the standardized residuals. The standardized residuals explain how much one observed value differed from what was expected divided by its standard error. If the absolute value of the standardized residual is greater then 2.0 , then it is a contributing factor to the overall significant chi-square test result (McGrew and Monroe 2000). If the results are insignificant, then we can 
conclude that the maps presented data equally well for students to perform the specified task. The chi-square test, in addition to descriptive statistics, will provide answers to my research questions and allow me to make conclusions from the results. 


\section{CHAPTER 4:}

\section{RESULTS}

This chapter presents results to describe performance on each of the four map reading tasks. Tables and graphs illustrate summary statistics, providing a numerical and visual overview of the data collected from the students' surveys. Inferential statistical tests identify if there are performance results that statistically deviate from expected performance for each map reading task that may allow us to conclude one map's data presentation makes it easier or more difficult for a specific task. Overall findings are presented as an additional measure of a map's ability to display information for spatial data analysis.

In order to properly compare results of students' performances, it must first be determined if one map presented students with more overall problems. One indication that a map might be more problematic is if students spend more time answering questions for one map type than another. To investigate any differences, I analyzed the time it took students in each map group to complete their surveys (Table 5 and Figure 11). A one-way ANOVA test revealed no statistically significant differences between map groups, $F(2,197)=1.23, p>.05$.

The ANOVA test for completion time indicates there were no major problems that arose in one map type that inhibited students' performance. This finding allows us to fairly compare students' performances between map groups. 
Table 5. Completion time summary statistics. Although the times (in seconds) do differ between groups, there is not a statistically significant difference between them to suggest one map was more difficult for students than another. Two packets did not have a recorded time and thus were not included in these results.

\begin{tabular}{c|c|c|c|c|c|c} 
Map Type & \multicolumn{1}{c}{$\begin{array}{c}\text { Mean } \\
\text { Time }\end{array}$} & $\begin{array}{c}\text { Median } \\
\text { Time }\end{array}$ & $\begin{array}{c}\text { Std. } \\
\text { Dev. }\end{array}$ & \multicolumn{1}{c}{$\begin{array}{c}\text { Min. } \\
\text { Time }\end{array}$} & \multicolumn{1}{c}{$\begin{array}{c}\text { Max. } \\
\text { Time }\end{array}$} & Range \\
\hline Choropleth & 677 & 670 & 172 & 288 & 1260 & 972 \\
\hline Dot & 637 & 621 & 159 & 368 & 1032 & 664 \\
\hline Graduated & 639 & 630 & 172 & 315 & 1130 & 815 \\
\hline
\end{tabular}

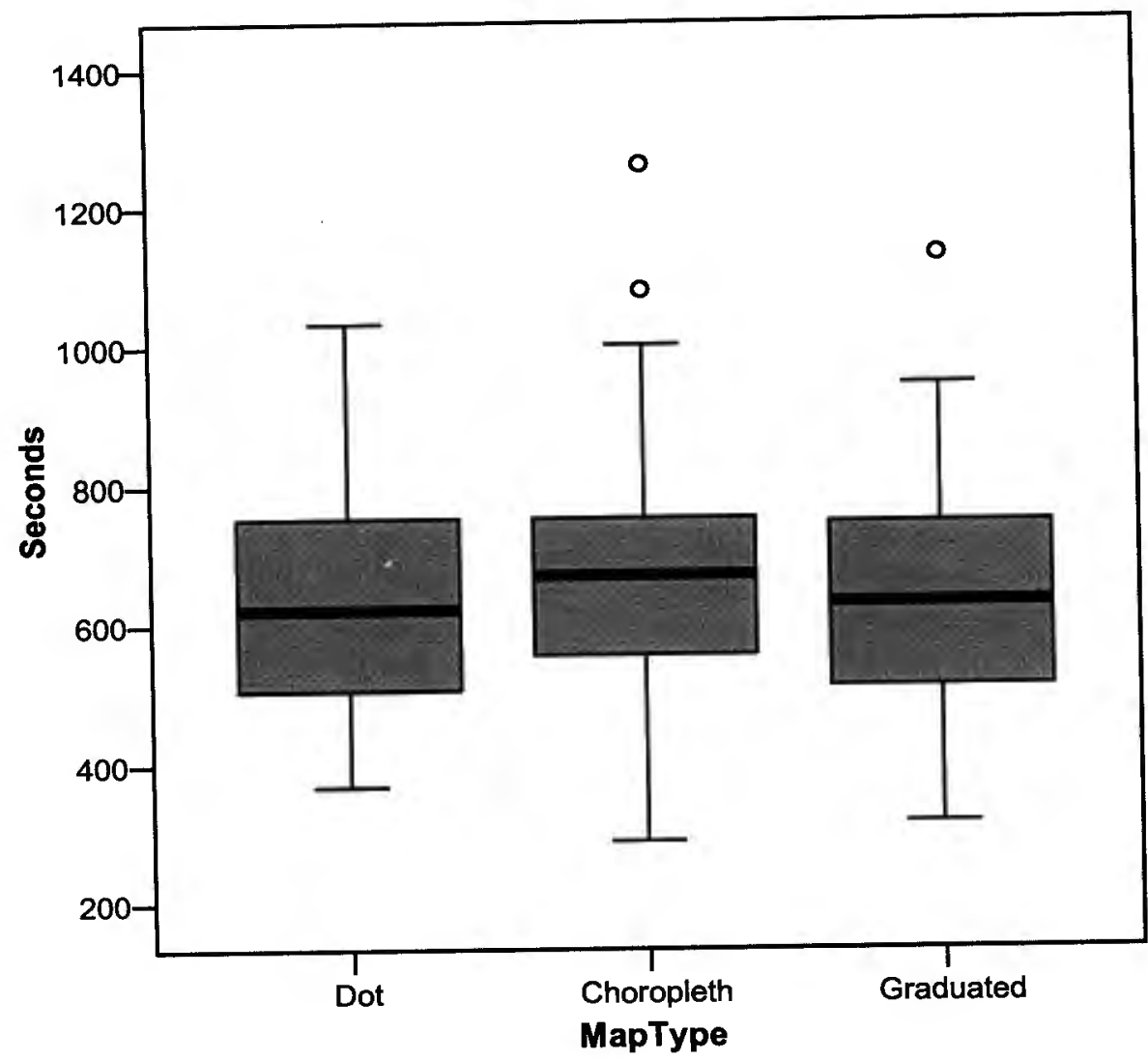

Figure 11. Box and whisker plots of map completion time. Each of the three box and whisker plots represents the entire spread of the data, while the three dots represent cases that SPSS recognized as outliers. Both the interquartile range (represented by the gray boxes) and the mean (represented by the black line inside the boxes) are similar for all three map types. 


\section{Questions 1 and 3: Interpreting the Symbolization}

1. On the map at the right there is a highlighted county. Find the same county in the map above. According to the map above, how many people would you estimate live in that county?

3. On the map at the right, look at the highlighted County $A$ and County $B$. Find these counties in the map above. Looking at the map above, which county has more people: county $A$ or county $B$ ?

Questions 1 and 3 test if students can interpret data by using the map key. Question 1 instructs students to determine the exact amount of people that live in Wheeler County, OR and then write their answer on the line provided for them. I determined if their answers fell within a range of correct values. The range includes any answer plus or minus 100 people of the number of people who reside in Wheeler County, OR (1,547 people). This value range was used because if the limits were expanded to a range such as plus or minus 500 people of the correct answer, values could include answers from students who misread an extra dot on the dot map (one dot $=500$ people).

A problem arose with the choropleth map responses to this question that made data interpretation and analysis tricky. Many students responded with the answer of simply "0-15." Without a label, one could interpret this many ways:

a) the student thinks 0 to 15 people live in the respective county, instead of $0-15$ people per square mile (in other words, the student doesn't understand the symbolization is a ratio, not a raw count) 
b) the student knew to match the county's hue to the hue in the map key, but was simply copying the key because he/she does not understand the symbology.

c) the student was abbreviating the answer and left off "people per square mile" but still understands the symbolization is a ratio for each square mile within the respective county.

Although it is impossible to tell without additional study, I developed a proxy to determine what the student meant by examining Question 6 responses that asked students to explain how the map worked. If a student explained in Question 6 that units of the map represented density, the " $0-15$ " answers could be counted correct for Question 1.

This is not the perfect solution because some students in each map group who answered Question 1 correctly did not explain their symbolization correctly for Question 6. However, the majority of those who correctly answered Question 1 also correctly answered Question 6. Without retesting all the students with additional questions, this method can serve as a proxy to clarify the choropleth Question 1 answers of " $0-15 . "$

Out of the 33 students who simply answered " $0-15$ " for Question 1, there was only one student who I counted correct because this student provided the following answer: "Because people live per mile so its shaded that because every square mile is about a family are present." The remaining 32 of the 33 students did not explain that the units represented density and their answers to Question 1 
were thus considered incorrect. The dot map and the graduated circle map answers to Question 1 did not have any comparable reoccurring complications.

Once I solved that data interpretation problem, I was able to plot the range of responses for each map displayed in Figures 12, 13, and 14. Figures 12, 13, and 14 illustrate that the data obtained from this question differ between map types. The choropleth map answers cluster around 0 , or an incorrect answer. The answers to the dot map cluster near the correct answer of 1,500, and the graduated circle map answers mostly cluster considerably above the correct answer. The summarized version of these figures are presented in Figure 15. 
CHOROPLETH Map Question 1:

How many people live in this county?

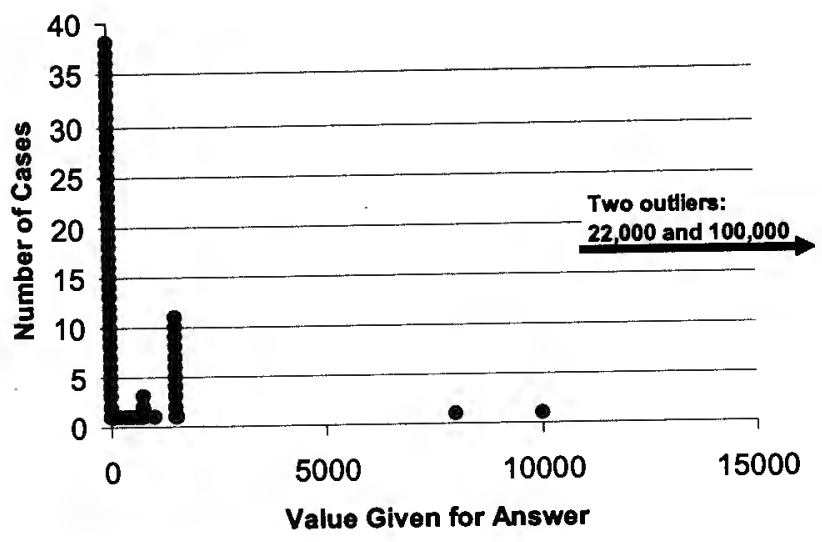

Figure 12. Distribution of answers given for Question 1 on the choropleth map (correct answer is $+/-100$ of 1500 people). For the sake of this graph, those who wrote " $0-15$ " were entered as " 0 " because they were incorrect answers. Those who wrote " $0-15$ people per square mile" were marked as recording " 1500 " since they are the same amount.
DOT Map Question 1:

How many people live in this county?

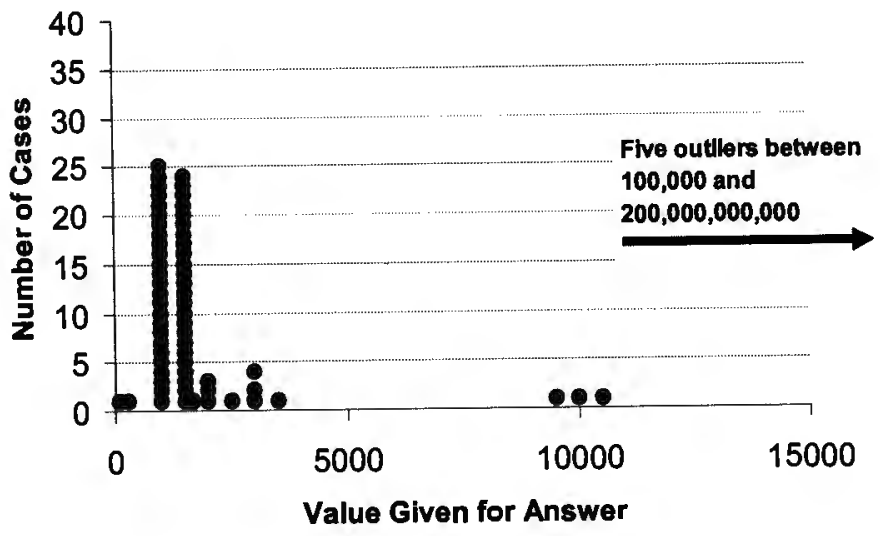

Figure 13. Distribution of answers given for Question 1 on the dot map (correct answer is $+/-100$ of 1500 people).
GRADUATED Circle Question 1:

How many people live in this county?

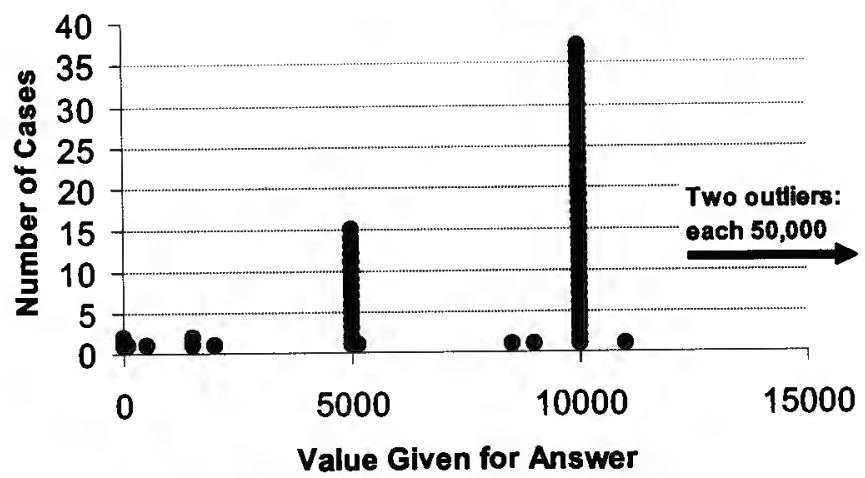

Figure 14. Distribution of answers given for Question 1 on the graduated circle maps (correct answer is + /100 of 1500 people). Note that the two values recorded as " 0 " were actually a) "very cold" and b) no answer. 
Question 1: How many people live in this county?

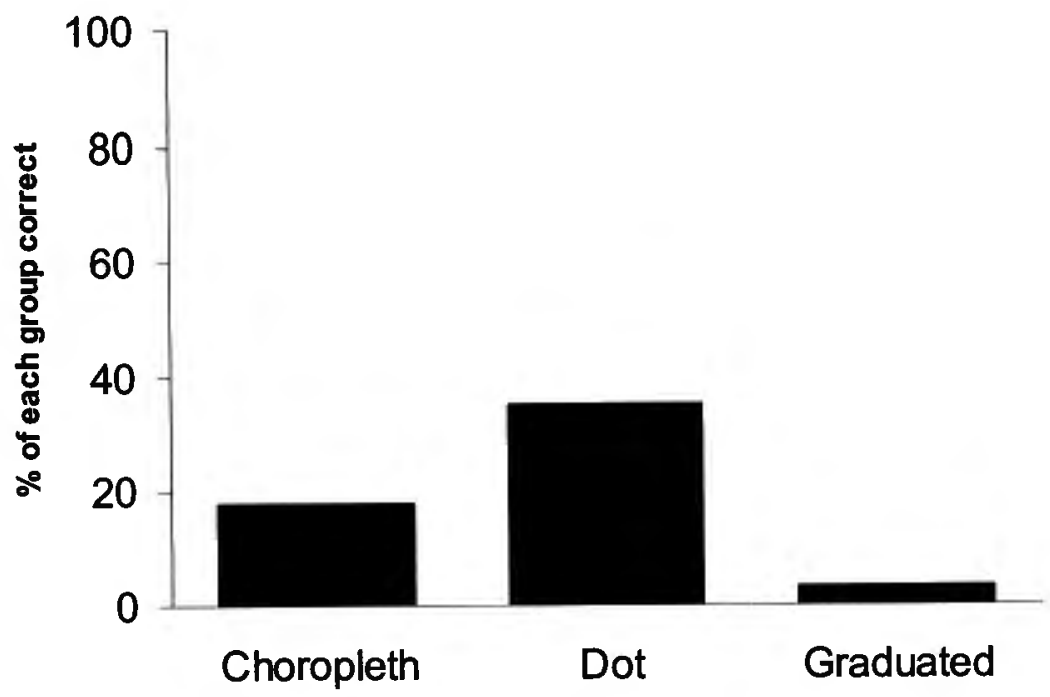

Figure 15. Correct answers to Question 1.

The bar graph in Figure 15 shows that very few students from each group could correctly extract population values from a single county. Additionally, compared to the dot map, the graduated circle map has a much lower percentage of students able to correctly identify that Wheeler County has a population of 1,500 people.

A Pearson's chi-square test on the counts of correct and incorrect answers for each map group produced statistically significant results, $\chi^{2}(2,202)=21.6$, $\mathrm{p}<.05$ (Table 6). This reveals that students' performance on interpreting symbologies in Question 1 statistically deviates from what is expected by chance. Additionally, the standardized residuals allow us to conclude that students performed statistically better than expected on the dot map, statistically worse than expected on the graduated circle map, and did not deviate from what was expected on the choropleth map. In other words, the dot map's symbology 
Table 6. Chi-square contingency table for Question 1.

\begin{tabular}{clrrr} 
Map Type & & Incorrect & Correct & Total \\
\hline \multirow{4}{*}{ Choropleth } & Expected Count & 54.9 & 13.0 & 68 \\
& Observed Count & 55.0 & 13.1 & 68 \\
& Residual & 0.1 & -0.1 & \\
\cline { 2 - 5 } & Std. Residual & $\mathbf{0 . 0}$ & $\mathbf{0 . 0}$ & \\
\hline \multirow{4}{*}{ Dot } & Expected Count & 55.7 & 13.3 & 69 \\
& Observed Count & 45.0 & 24.0 & 69 \\
& Residual & 10.7 & -10.7 & \\
\cline { 2 - 5 } & Std. Residual & $\mathbf{- 1 . 4}$ & $\mathbf{2 . 9}$ & \\
\hline \multirow{4}{*}{ Graduated Circle } & Expected Count & 52.5 & 12.5 & 65 \\
& Observed Count & 63.0 & 2.0 & 65 \\
& Residual & -10.5 & 10.5 & \\
\cline { 2 - 5 } & Std. Residual & $\mathbf{1 . 5}$ & $\mathbf{- 3 . 0}$ & \\
\hline & \multicolumn{1}{c}{ Total } & 163 & 39 & 202
\end{tabular}

presented data better than expected by chance and the graduated circle's symbology presented data worse than expected for the task of extracting exact values.

Question 1's results must be considered with the results of Question 3 to ascertain if students can interpret symbology. The second task (Question 3 ) tests students on their abilities to compare data by instructing students to identify if County A or County B has a higher population. This task requires students to interpret symbology, but on an ordinal scale, meaning they only have to determine which county has a higher population than the other.

All students marked an " $\mathrm{X}$ " in either County A (the correct answer) or County B. The percentage of students in each group recording County A as their response are presented in Figure 16. The bar graph reveals that many students 
Question 3: Does County A or County B have a

higher population?

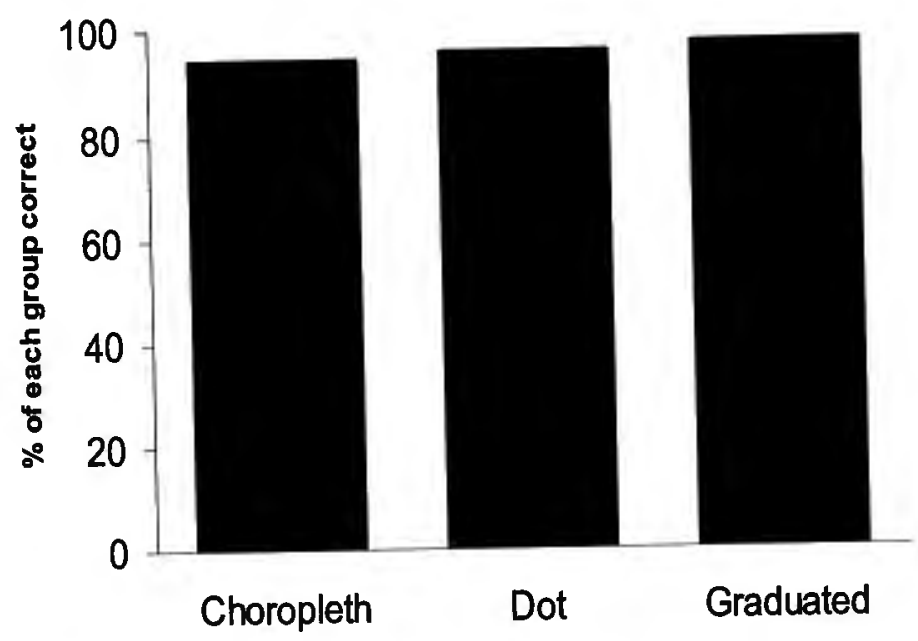

Figure 16. Correct answers to Question 3.

from each group were able to correctly identify that Linn County (County A) had a higher population than Grant County (County B). Each map group performed very well, with over 90 percent of all students able to interpret the symbology to conclude that County A had a higher population.

Students' performance on interpreting symbologies in Question 3 showed no statistically significant deviance from performance expected by chance as determined by a Pearson's chi-square test, $\chi^{2}(2,202)=2.25, \mathrm{p}>.05$ (Table 7). In other words, each map's symbology presented data equally well to allow students to compare population values.

To summarize students' abilities to interpret symbology, students performed poorly on Question 1 that required them to determine exact population values for Wheeler County. A Pearson's chi-square test concluded that dot map's 
Table 7. Chi-square contingency table for Question 3.

\begin{tabular}{|c|c|c|c|c|}
\hline Map Type & & Incorrect & Correct & Total \\
\hline \multirow{4}{*}{ Choropleth } & Expected Count & 4.4 & 63.6 & 68 \\
\hline & Observed Count & 6.0 & 62.0 & 68 \\
\hline & Residual & 1.6 & -1.6 & \\
\hline & Std. Residual & 0.8 & -0.2 & \\
\hline \multirow{4}{*}{ Dot } & Expected Count & 4.4 & 64.6 & 69 \\
\hline & Observed Count & 2.0 & 67.0 & 69 \\
\hline & Residual & -2.4 & 2.4 & \\
\hline & Std. Residual & -1.2 & 0.3 & \\
\hline \multirow{4}{*}{ Graduated Circle } & Expected Count & 4.2 & 60.8 & 65 \\
\hline & Observed Count & 5.0 & 60.0 & 65 \\
\hline & Residual & 0.8 & -0.8 & \\
\hline & Std. Residual & 0.4 & -0.1 & \\
\hline & Tota & 13.0 & 189.0 & 202 \\
\hline
\end{tabular}

symbology presents data better for students to extract exact population values.

Additionally, students performed very well on Question 3 where they determined which of two counties had a higher population. A Pearson's chi-square test revealed that the maps present data equally well for this task.

\section{Question 2: Making Inferences}

2. Mark an $X$ in one county on the map at the top of the page that you think has a city with many people living in it. There may be more than one correct answer, but only put an $X$ in one county.

Question 2 asks students to identify a county that probably has a large city in it. They must understand that a large city would most likely be found in a county with a high population. To answer this question, students marked an " $\mathrm{X}$ " on the map in their chosen county. All counties that had a city with a population over 
50,000 people were counted correct. Correct answers include Multnomah, Lane, Marion, Washington, Jackson, and Deschutes Counties. The summation of students' answers are displayed in Figure 17. As the Figure illustrates, students most frequently chose Multnomah County as the county most likely to have a city in it.

\section{Question 2: Which county probably has a city in it?}

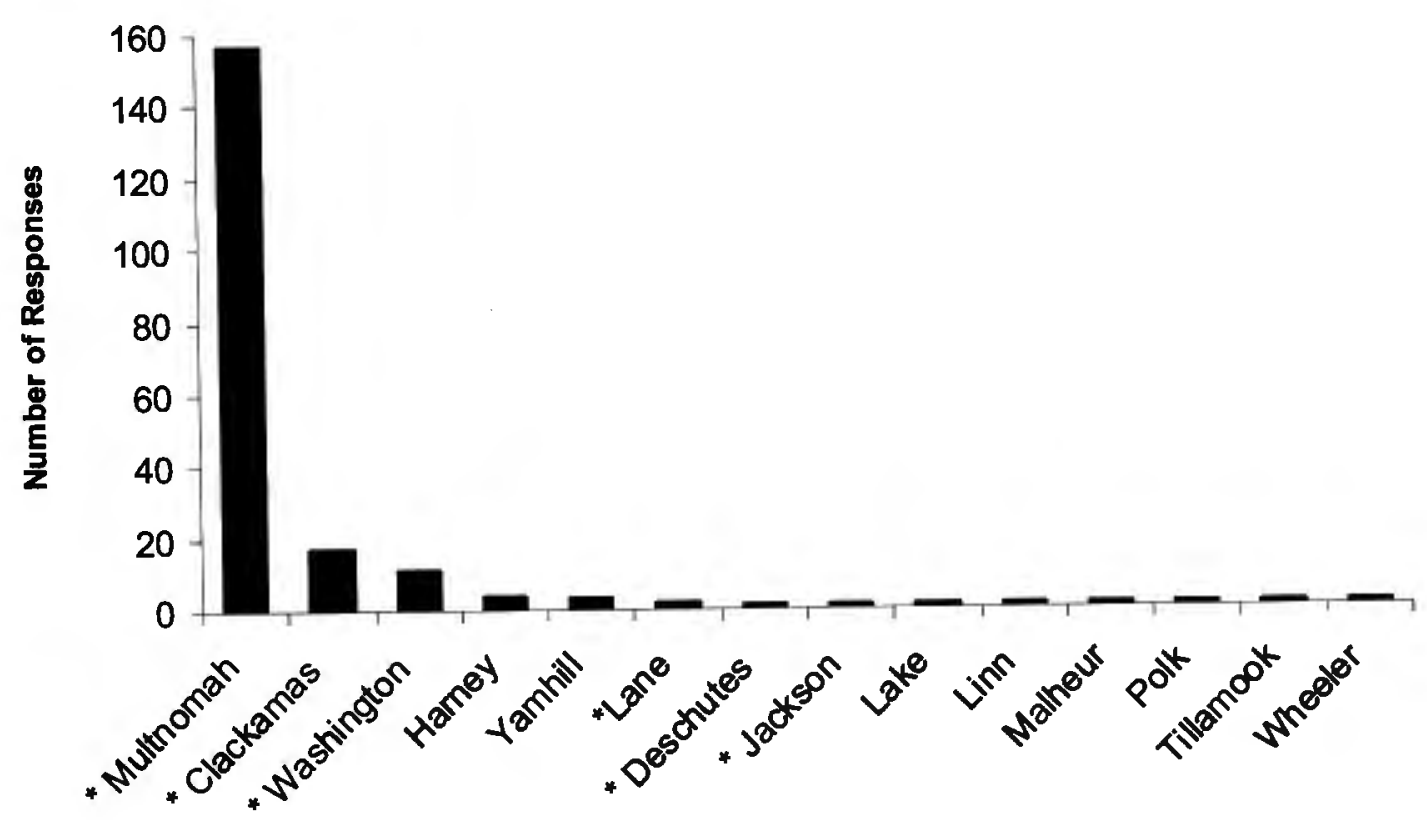

Figure 17. Responses to Question 2. Counties with an asterisk $\left(^{\star}\right)$ indicate possible correct answers.

Most students performed well on this task as seen in Figure 18. The bar graph depicts a high percentage of students from each map group able to identify a county that would probably have a large city in it.

A Pearson's chi-square test reveals that the number of students to correctly make inferences in Question 2 did not statistically deviate greater than expected 
by chance, $\chi^{2}(2,202)=1.05, p>.05$ (Table 8$)$. In other words, the chi-square result indicates that each map displayed data equally well to allow students to make inferences.

Question 2: Which county probably has a city in it?

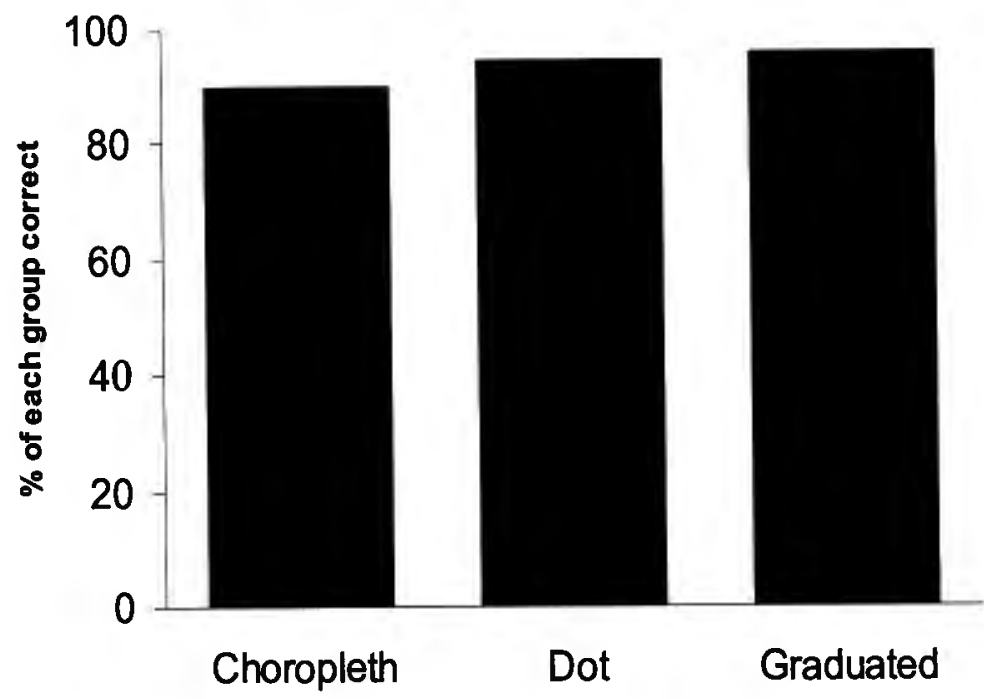

Figure 18. Performance on Question 2.

Table 8. Chi-square contingency table for Question 2.

\begin{tabular}{clrrr} 
Map Type & & Incorrect & Correct & Total \\
\hline \multirow{3}{*}{ Choropleth } & Expected Count & 4.4 & 63.6 & 68 \\
& Observed Count & 6.0 & 62.0 & 68 \\
& Residual & 1.6 & -1.6 & \\
\cline { 2 - 5 } & Std. Residual & $\mathbf{0 . 8}$ & $-\mathbf{0 . 2}$ & \\
\hline \multirow{3}{*}{ Dot } & Expected Count & 4.4 & 64.6 & 69 \\
& Observed Count & 4.0 & 65.0 & 69 \\
& Residual & -0.4 & 0.4 & \\
\cline { 2 - 5 } & Std. Residual & $-\mathbf{0 . 2}$ & $\mathbf{0 . 1}$ & \\
\hline \multirow{4}{*}{ Graduated Circle } & Expected Count & 4.2 & 60.8 & 65 \\
& Observed Count & 3.0 & 62.0 & 65 \\
& Residual & -1.2 & 1.2 & \\
\cline { 2 - 5 } & Std. Residual & $-\mathbf{0 . 6}$ & $\mathbf{0 . 2}$ & \\
\cline { 2 - 5 } & \multicolumn{1}{c}{ Total } & 13.0 & 189.0 & 202
\end{tabular}




\section{Question 4: Spatial Pattern Recognition}

3. Geographers say that a region is an area that includes more than one county. A region does not have to follow county borders. Many people see regions of high, medium, and low population on the map at the top of the page. On the map at the top of the page, draw lines around the regions that you think are high, medium, and low and label each with the word "High," "Medium," or "Low." When you get done, every place in Oregon should be in a high, medium, or low region.

Question 4 tests students' abilities to identify regions, requiring them to interpret data values and group similar values. For students' answers to be counted correct, the map on which they drew their response had to meet all three of the following criteria: a) a line/circle had to be drawn around an area and labeled, b) each identified region had to be labeled correctly, and c) at least $75 \%$ of the data on the map had to be categorized into regions. If students incorrectly labeled regions or simply labeled each county without drawing boundaries, I did not count it as correct. Examples of "correct" and "incorrect" region drawings are displayed in Figure 19. 
Correct
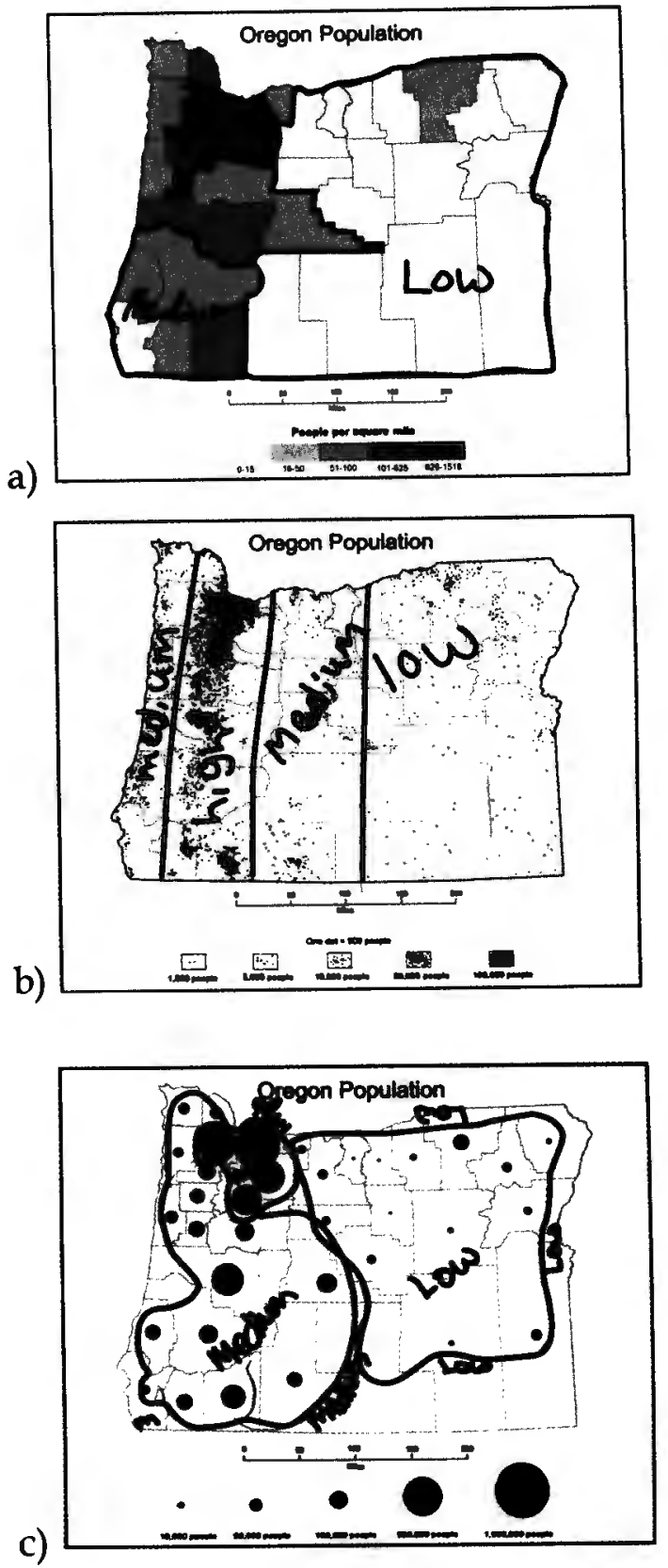

\section{Incorrect}

d)

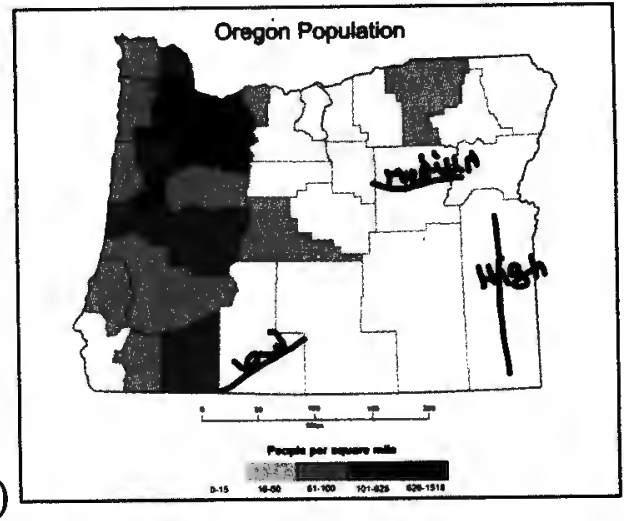

e)

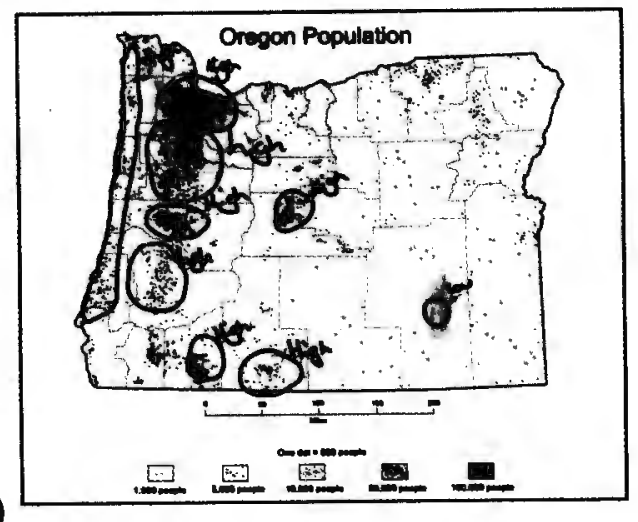

f)

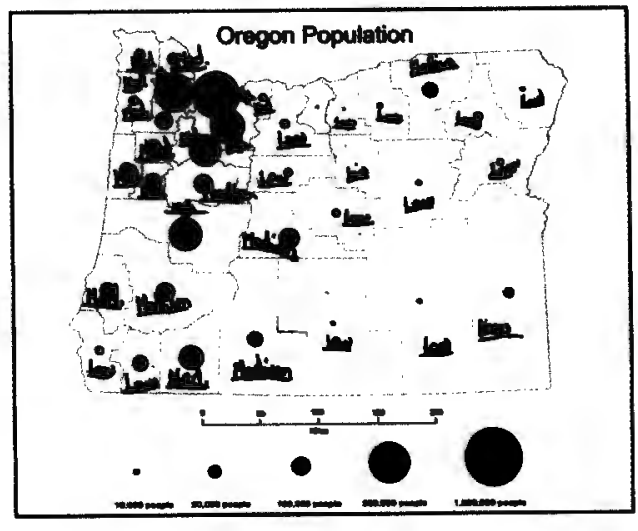

Figure 19. Examples of Question 4 answers. Examples $a, b$, and $c$ illustrate correct responses for each type of map. Example $d$ is incorrect because all high, medium, and low labels are on counties of the same population density value. Example $e$ is incorrect because less than half the state is in a region and the student only circled areas of high population. Example $f$ is incorrect because the student only labeled each county, but did not draw any region boundaries. 
The percentage of students who could correctly draw a region for each map type is presented in Figure 20.

Question 4: Draw Regions of high, medium, and low

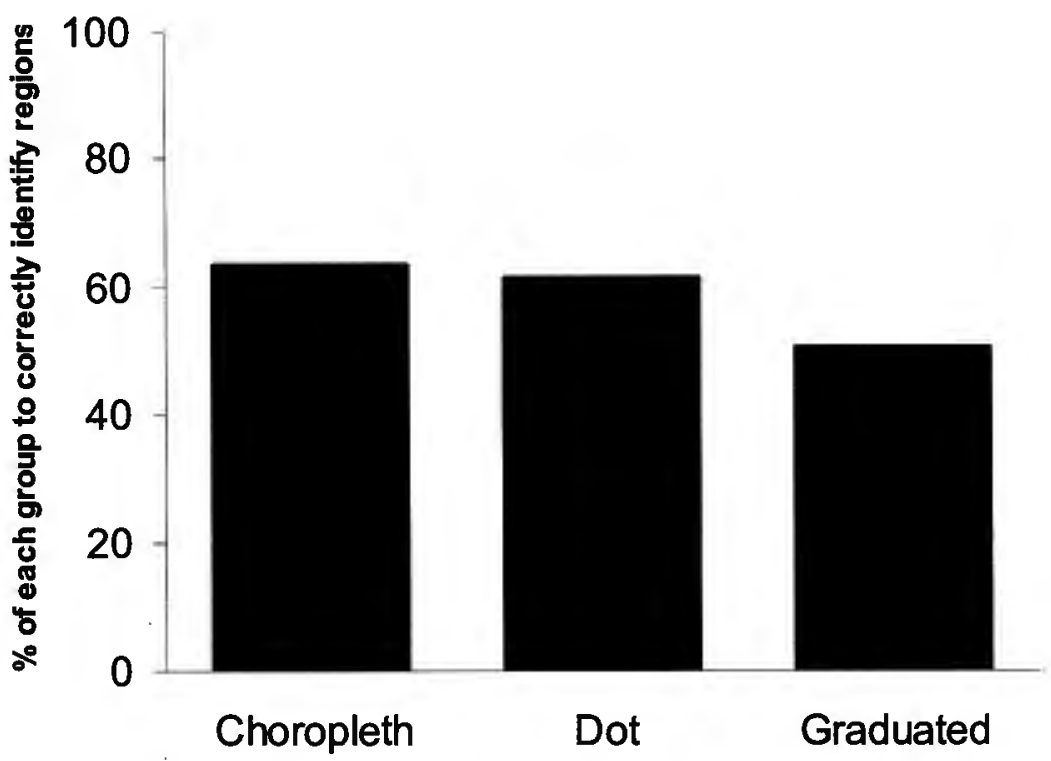

Figure 20. Correct responses to Question 4

Between one-half and two-thirds of each group was able to group similar values into regions. A Pearson's chi-square test shows no statistically significant deviances of correct and incorrect answers for this region-drawing task from what is expected by chance, $\chi^{2}(2,202)=2.68, p>.05$ (Table 9). To summarize, students performed moderately well on this task and each map displayed data equally well to allow students to categorize data into regions. 
Table 9. Chi-square contingency table for Question 4

\begin{tabular}{clrrr}
\hline Map Type & & Incorrect & Correct & \multicolumn{1}{c}{ Total } \\
\hline \multirow{3}{*}{ Choropleth } & Expected Count & 27.9 & 40.1 & 68 \\
& Observed Count & 26.0 & 42.0 & 68 \\
& Residual & -1.9 & 1.9 & \\
\cline { 2 - 5 } & Std. Residual & $-\mathbf{0 . 4}$ & $\mathbf{0 . 3}$ & \\
\hline \multirow{3}{*}{ Dot } & Expected Count & 28.4 & 40.6 & 69 \\
& Observed Count & 25.0 & 44.0 & 69 \\
& Residual & -3.4 & 3.4 & \\
\cline { 2 - 5 } & Std. Residual & -0.6 & $\mathbf{0 . 5}$ & \\
\hline \multirow{3}{*}{ Graduated Circle } & Expected Count & 26.7 & 38.3 & 65 \\
& Observed Count & 32.0 & 33.0 & 65 \\
& Residual & 5.3 & -5.3 & \\
\cline { 2 - 5 } & Std. Residual & $\mathbf{1 . 0}$ & $-\mathbf{0 . 9}$ & \\
\hline & & 83.0 & 119.0 & 202
\end{tabular}

\section{Question 5: Thinking Geographically}

Question 5 asked students to generate two questions about the distribution of the data, a skill necessary to becoming a geographically-informed person as described by the National Geography Standards. I determined if a question was correct or incorrect based on certain criteria. A question was correct if it asked about the distribution of the data or a pattern recognizable in the data but not explained by the data. Examples of correct responses include:

a) How come so many people live by the coast?

b) Why is the west side so popular?

c) Why does nobody live in the east?

d) Why do more people live on the western side of Oregon instead of the eastern?

e) In the more populated counties, what makes it appealing?

f) Is the population going up or down?

g) Why are there more people in smaller counties?

h) Why do people live in the West/NW Oregon?

i) What is it like living in a very high populated county?

j) Why is there so much land and not many people to use? 
k) What kind of geographic features make Western Oregon more desirable to live than in eastern Oregon?

l) How do so many people live in such a small place?

m) Is Oregon's population like this because of the way the land is?

n) What percentage of Oregon's population are girls?

o) How does the climate affect the population in that region?

p) How come countries that are large have small population?

q) Why is there on area in the eastern side that has 16-50 people per square mile instead of 0-15 like everywhere surrounding it?

A question was incorrect if it was: a) a geographic question already asked in the test booklet and that can be derived from the map; b) a question that asked about symbology and not distribution; or c) an incorrect answer/no answer. Examples of these three incorrect categories are:

Questions that can be answered from the map:

a) Which county has the highest population in Oregon?

b) Which counties are most populated?

Questions that deal with symbology:

c) The one dot is big and goes over other counties, do these counties have 1,000,000 people also?

d) Why are there so many dots spread out?

Other Incorrect Answers:

e) I don't know

f) Is there any eagles in Oregon?

g) Why is Oregon green?

h) Why aren't the counties labeled?

Table 10 provides the division of kinds of both correct and incorrect answers that students provided. Most students, regardless of map type that they analyzed, were able to make inquiries about data distributions (Figure 21). 
Table 10. Responses to Question 5. The first column describes the percentage of each map group that correctly provided a geographic question. The remaining two columns describe raw counts of types of incorrect answers.

\begin{tabular}{r|r||cc}
\hline & $\begin{array}{c}\text { Correct } \\
\text { Answers }\end{array}$ & \multicolumn{2}{c}{ Incorrect Answers } \\
\hline \hline Map Type & $\begin{array}{c}\text { At least one } \\
\text { correct } \\
\text { geographic } \\
\text { question }\end{array}$ & $\begin{array}{c}\text { \# of total } \\
\text { answers that are } \\
\text { geographic, but } \\
\text { already asked }\end{array}$ & $\begin{array}{c}\text { \# of total } \\
\text { answers that } \\
\text { inquired about } \\
\text { symbology }\end{array}$ \\
\hline Choropleth & $82.3 \%$ & 7 & 4 \\
\hline Dot & $71.0 \%$ & 20 & 2 \\
\hline Graduated & $69.2 \%$ & 19 & 2 \\
\hline
\end{tabular}

Correct responses to Question 5: Ask a geographic question

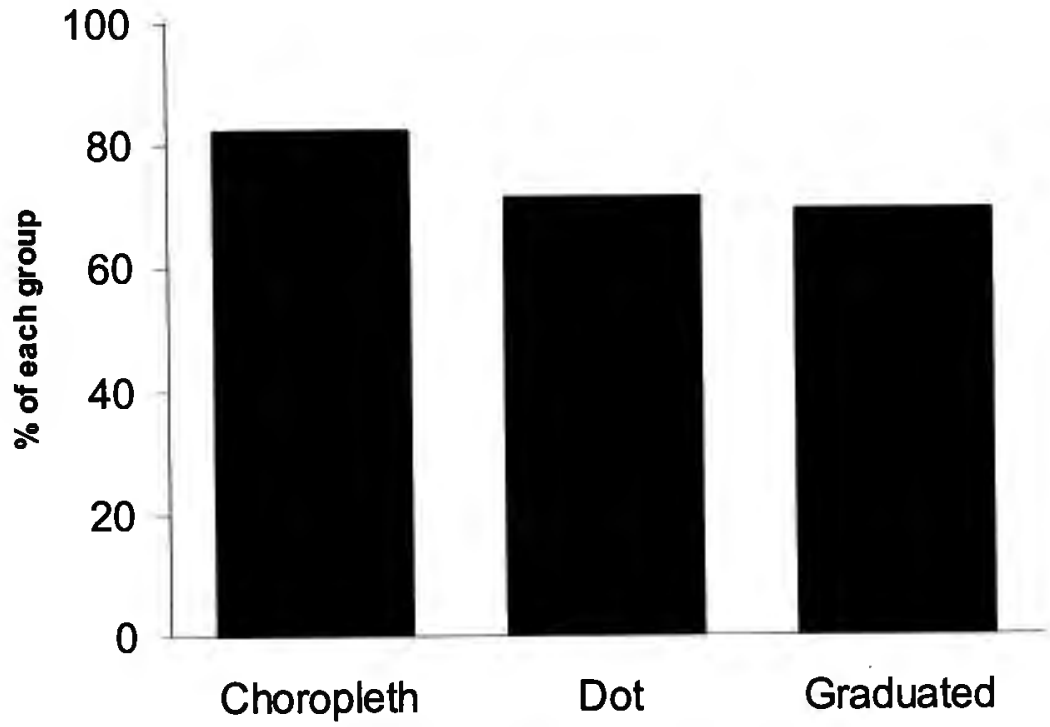

Figure 21. Percentage of correct responses to Question 5.

Of those who did not provide a correct answer, many simply repeated already asked questions. These were considered incorrect because the instructions state 
that students' questions should help them gain understanding of what they see in the data distribution. Nevertheless, about 70 percent of each group was able to inquire about causations or ramifications of data distributions.

In addition, a Pearson's chi-square test allows us to conclude that the number of students who were able to ask a geographic question and those who were not were not statistically different than was expected by chance, $\chi^{2}(2,202)=$ $3.57, \mathrm{p}>.05$ (Table 11). Students performed well at making inquiries about data distributions and the maps presented data equally well for students to complete this task. To summarize, students performed well at asking geographic questions and a chi-square test confirmed that each map displays data equally well to allow students to make inquiries about data distributions.

Table 11. Chi-square contingency table for Question 5.

\begin{tabular}{clrrr} 
Map Type & & Incorrect & Correct & Total \\
\hline \hline \multirow{4}{*}{ Choropleth } & Expected Count & 17.5 & 50.5 & 68 \\
& Observed Count & 12.0 & 56.0 & 68 \\
& Residual & -5.5 & 5.5 & \\
\cline { 2 - 5 } & Std. Residual & -1.3 & $\mathbf{0 . 8}$ & \\
\hline \multirow{4}{*}{ Dot } & Expected Count & 17.8 & 51.2 & 69 \\
& Observed Count & 20.0 & 49.0 & 69 \\
& Residual & 2.2 & -2.2 & \\
\cline { 2 - 5 } & Std. Residual & $\mathbf{0 . 5}$ & $-\mathbf{0 . 3}$ & \\
\hline \multirow{4}{*}{ Graduated Circle } & Expected Count & 16.7 & 48.3 & 65 \\
& Observed Count & 20.0 & 45.0 & 65 \\
& Residual & 3.3 & -3.3 & \\
\cline { 2 - 5 } & Std. Residual & $\mathbf{0 . 8}$ & -0.5 & \\
\hline & \multicolumn{1}{c}{ Total } & 52.0 & 150.0 & 202
\end{tabular}




\section{Question 6: Understanding the Symbolization}

Each map groups' Question 6 was different because it required students to explain how the map works. A student's response was considered correct if he or she correctly identified the way the symbol showed the data. For example, on the choropleth map, correct answers included explanations such as:

a) All of the map is shaded because it is showing the average and the map would be very confusing to read if the parts of the map were there and forests, lakes, and rivers were just white.

b) The legend is telling how many people live in each square mile. It's the average.

c) The map shows an average of the area, so if the whole class in one comer and the teacher is in another the average would be something like 5 people for every square feet.

On the dot map, explanations such as the following were considered correct:

a) Because one dot is equal to 500, so if there is no dot but people still live there, there are less than 500 people in that place.

b) The dot represents the population, not the location of each individual.l

c) When there is a dot it probably symbolizes 500 in that area. If an area has only 250 people you can't put a full dot there.

On the graduated circle map, answers that were considered correct included explanations such as:

a) Because it's showing how many people live in the county.

b) Because the circle is just showing how many people are in each region, not how many people live in the shadow of the circle.

c) The circles tell you the population, not where people live because if it did there would be dots almost everywhere. 
Certain examples for incorrect answers included explanations such as:

a) Because people can live around those areas like on house boats on lakes, or people can live in forests. And the map is only estimating.

b) There are records of where people live, bought the house and they can track you down easier.

c) Well, the map is correct depending on when they took the data. If they took the data in example $=1999$ it's been a couple of years since that year. Most of the forest has been chopped down. And the population has grown since then.

d) Maybe because not many people live there because it's not a really good place to live. Or maybe it's because they just want to live in the middle of nowhere.

e) Because you probably look on a computer.

$f$ Because it got data from the computer.

g) I have no idea

h) Maybe the mappers didn't count all the people in the area because that's hard to do.

Figure 22 summarizes the percent of each group that correctly explained the map symbolization and illustrates a poor performance on this task for students analyzing the choropleth and graduated circle maps. A Pearson's chi-square test produced statistically significant results, $\chi^{2}(2)=20.77, p<.05$ (Table 12). The standardized residuals reveal that students performed better on dot maps than expected and performed about as expected on the choropleth and graduated circle maps. To summarize, about one third to one half of each map group was able to explain their map's symbology. Additionally, the dot maps allowed students to explain how dot maps worked statistically better than would be expected compared to the symbolization of the choropleth and graduated circle maps. 
Correct responses to Question 6: Explain the map

symbology

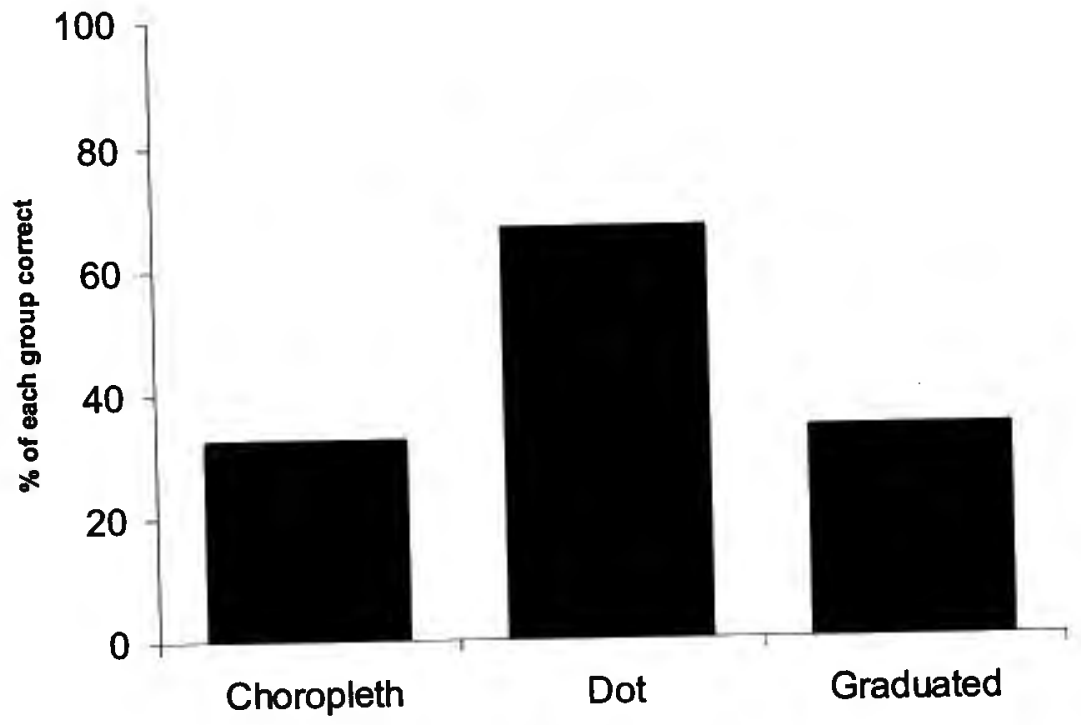

Figure 22. Percentage of correct responses to Question 6

Table 12. Chi-square contingency table for Question 6.

\begin{tabular}{clrrr} 
Map Type & & Incorrect & Correct & Total \\
\hline \multirow{3}{*}{ Choropleth } & Expected Count & 37.7 & 30.3 & 68 \\
& Observed Count & 46.0 & 22.0 & 68 \\
& Residual & 8.3 & -8.3 & \\
\cline { 2 - 5 } & Std. Residual & $\mathbf{1 . 4}$ & $\mathbf{- 1 . 5}$ & \\
\hline \multirow{3}{*}{ Dot } & Expected Count & 38.3 & 30.7 & 69 \\
& Observed Count & 23.0 & 46.0 & 69 \\
& Residual & -15.3 & 15.3 & \\
\cline { 2 - 5 } & Std. Residual & $\mathbf{- 2 . 5}$ & $\mathbf{2 . 8}$ & \\
\cline { 2 - 5 } & Expected Count & 36.0 & 29.0 & 65 \\
& Observed Count & 43.0 & 22.0 & 65 \\
\cline { 2 - 5 } Graduated Circle & Residual & 7.0 & -7.0 & \\
\cline { 2 - 5 } & Std. Residual & $\mathbf{1 . 2}$ & $\mathbf{- 1 . 3}$ & \\
\cline { 2 - 5 } & & 112.0 & 90.0 & 202
\end{tabular}




\section{Student Demographic Characteristics}

In order to see if students' age, gender, teacher, or class were associated with their performance, I calculated an overall performance score for each student (Figure 23).
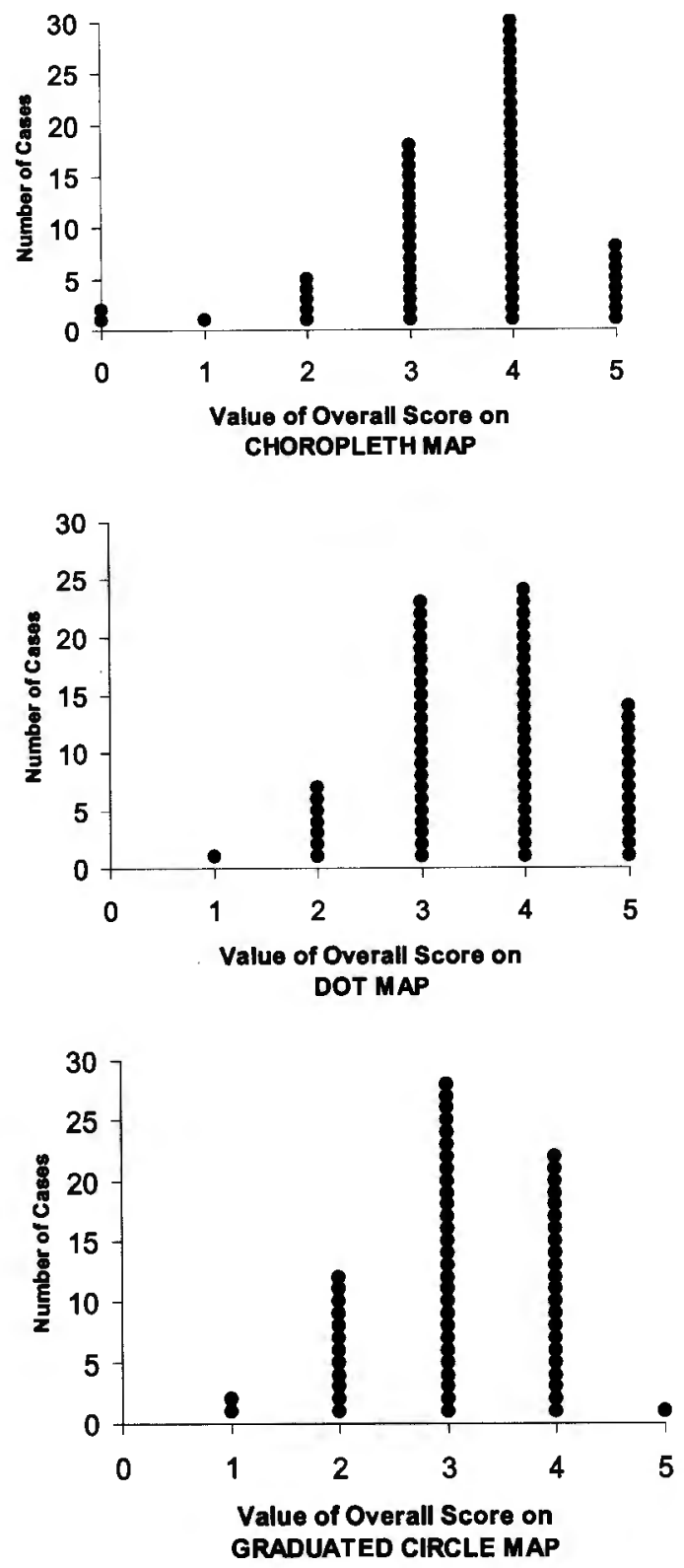

Figure 23. Overall performance by map group. 
A student's overall performance could rank from 0 (no answers given to Question 1 through 5 were correct) to 5 (all answers to Question 1 through 5 were correct). The figures illustrate that dot maps' overall scores cluster at the higher end of the range, the choropleth map scores cluster at the mid-to-upper end of the range, and the graduated circle overall scores cluster at the mid-range of scores. Table 13 displays percentages of overall scores and indicates that students performed better on the dot map and worse on the graduated circle map.

Table 13. Overall performance by map group. Values in each cell indicate percentage of their respective group.

\begin{tabular}{rrrr} 
Map Type & All Correct & $\begin{array}{r}\text { At least 4 } \\
\text { questions } \\
\text { correct }\end{array}$ & $\begin{array}{r}\text { At least 3 } \\
\text { questions } \\
\text { correct }\end{array}$ \\
\hline Choropleth & 11.8 & 55.9 & 82.3 \\
\hline Dot & 20.3 & 55.1 & 88.4 \\
\hline Graduated Circle & 1.5 & 35.4 & 78.4 \\
\hline
\end{tabular}

A Pearson's chi-square test determined that there is a statistically significant difference on performance across all tasks from what would be expected, $\chi^{2}$ (6, 202) $=16.21, \mathrm{p}<.05($ Table 14$)$. 
Table 14. Chi-square contingency table for overall scores. The overall scores of 0,1 , and 2 were combined into one column/score because these values were so low for all three map types.

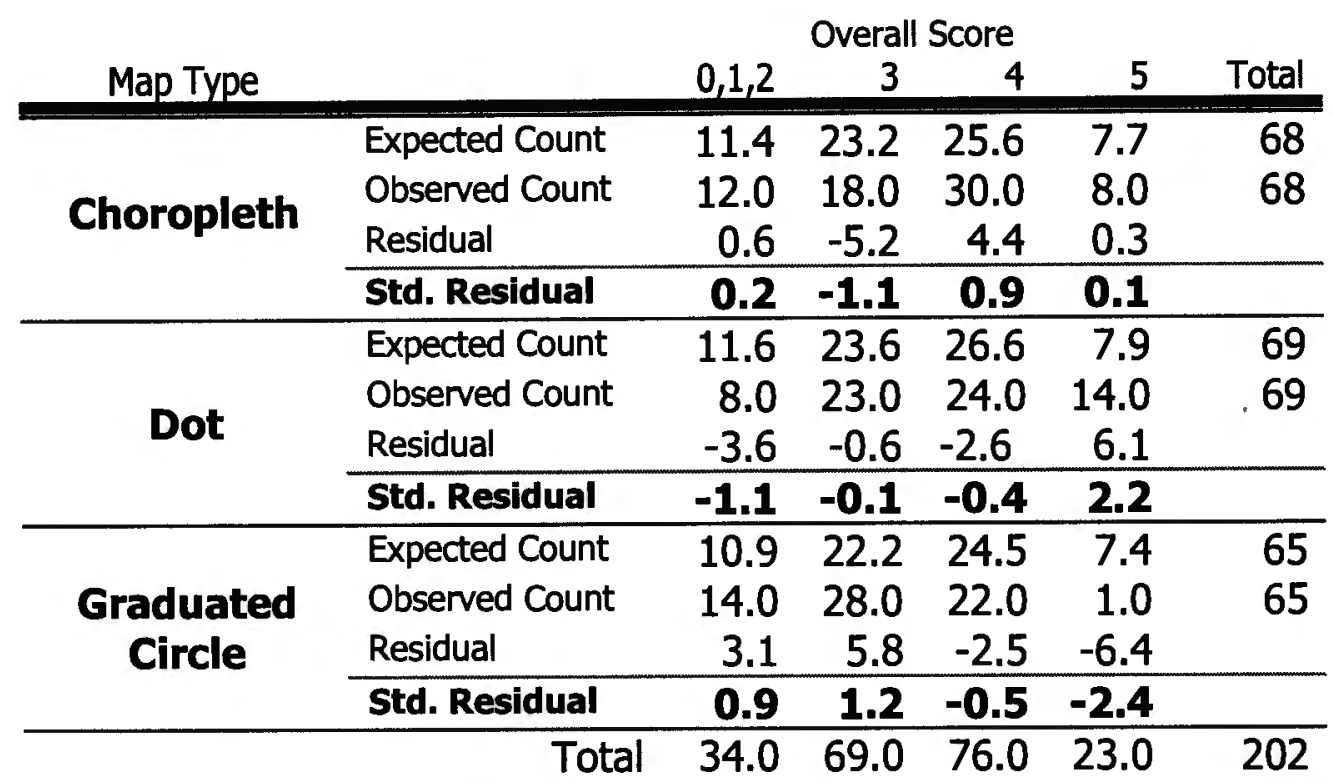

The standardized residuals reveal that students performed better than expected on dot maps and worse than expected on graduated circle maps, while performance on choropleth maps was not different than expected by chance.

I then ran a Pearson's chi-square test to see if overall performance on the survey and age, gender, teacher, or class were associated with each other. None of the test results were significant as reported in Table 15.

Table 15. Chi-square values of tests between characteristics and overall performance.

\begin{tabular}{crcc}
$\begin{array}{c}\text { Column } \\
\text { Variable }\end{array}$ & $x^{2}$ Value & df & $\begin{array}{c}\text { Significant } \\
\text { (at the .05 } \\
\text { level) }\end{array}$ \\
\hline \hline Teacher & 5.57 & 15 & No \\
Age & 12.41 & 15 & No \\
Gender & 9.47 & 5 & No \\
Class & 29.5 & 35 & No
\end{tabular}


Because this finding explains that students' overall performance score was independent of their teachers, ages, genders, or classes, it is easier to generalize this study's results to a broader population of sixth-graders.

\section{Chapter Review}

Each question in the survey required students to perform different map reading tasks that require different levels of skill. The ability to interpret symbolization required two steps. Between 91 percent and 97 percent of each map group was able to correctly tell which of two counties had the higher population (Question 3), but less than one third of each map group could correctly provide an exact value for a specified county's population with dot map readers doing better than expected on this question (Question 1). The students performed better on Question 2 that asked them to identify a county that probably has a large city in it, with between 90 percent and 95 percent of each group correctly making inferences from the map data. Students also were able to class similar data values into regions, with between 50 percent and 63 percent of each group correctly completing Question 4. Between 70 and 82 percent of each map group was able to inquire about patterns present in the data as illustrated from Question 5 results. When the students' overall scores were combined for the entire exercise, those analyzing the dot maps did statistically better than expected by chance and those reading the graduated circle maps did worse than expected. However, those who analyzed the choropleth maps performed no differently than expected by chance. Finally, overall performance was independent of a student's teacher, class, age, or 
gender; allowing these results to be more likely generalized to the entire population of sixth-graders. 


\section{CHAPTER 5:}

\section{ANALYSIS AND DISCUSSION}

The results indicate that students performed well, with the exception of Question 1, on the map-reading tasks. This chapter will discuss the results and explain if they provide support for or against the four hypotheses. It will also explore additional questions that the results uncovered about what children do and do not understand about the three thematic maps.

\section{Interpreting the Symbolization: Questions 1 and 3}

Interpreting the symbolization on maps is one of the first steps needed to understand the distributions they depict. Questions 1 and 3 test students on their abilities to execute these primary tasks and results provide support for Hypothesis 1:

\section{Sixth-grade students can interpret symbolization on graduated circle maps, dot maps, and choropleth maps.}

Hypothesis 1 is supported by the finding that between 91 percent and 97 percent of students in each map group correctly answered Question 3. They had to make decisions about which county had a higher population than the other and the results illustrate they can interpret map symbology. Additionally, all three map symbologies displayed data equally well for students to answer this question as revealed by the insignificant chi-square test result. However, the very low 
percentage of students able to correctly answer how many people live in Wheeler County (Question 1) suggests that students need additional work with these three map types to become proficient at thematic map reading.

Question 1 would appear to be a basic, if not simple, question. It requires students to interpret the map symbology using the map key to identify the number of people living in Wheeler County. Yet, the performance on this task yields two surprising results.

First, students performed very poorly on this task, with under one-third of each group obtaining the correct answer. One possible explanation for poor performance is that students may need additional work at extracting ratio-level values from maps. More students were able to correctly answer the ordinal data question (Question 3) than the question concerning exact values (Question 1). This seems logical because the abilities to use ratio data is taught to students after they grasp ordinal data concepts. For example, students are first taught to count (they understand the number 10 comes after the number 9 because it is larger) before they are taught to multiply and divide. However, because there were students who could correctly extract the answer of about 1,500 people from the maps' symbologies to answer Question 1 in addition to the many students who could answer Question 3, the low score on this task may mean that students need additional practice with data extraction. Additionally, it may mean that there is a better explanation for poor performance on Question 1. 
Another explanation of such a poor performance on this task might stem from problems understanding how to interpret and apply the map key. As illustrated in Figure 24, map keys do not display every data value found on the

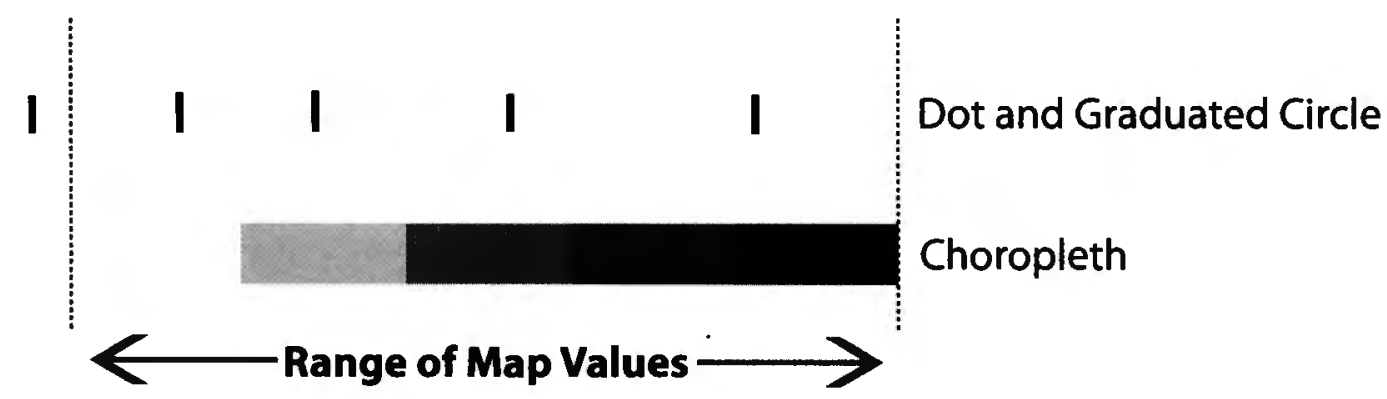

Figure 24. Range of map values covered by each map legend. The dot and graduated circle map keys only provide five examples of the continuous range of values found on the map, while the choropleth map key covers all values displayed on the map.

map unless it is a choropleth map. In the dot and graduated circle maps, students are only given five values in the wide range of map values and are expected to extrapolate or interpolate values to determine the majority of the values found in the map data.

A high percentage of students recorded the lowest value provided in the graphic map key as the answer to the question (Figure 25 and Table 16). This may indicate that students approach map data interpretation as a multiple choice task. In other words, students expect the map key to contain all values that are present on the map. Because there are so many values presented in dot maps and graduated circle maps, rarely do the graphic map keys show every single data 


\section{Choropleth Map Key}

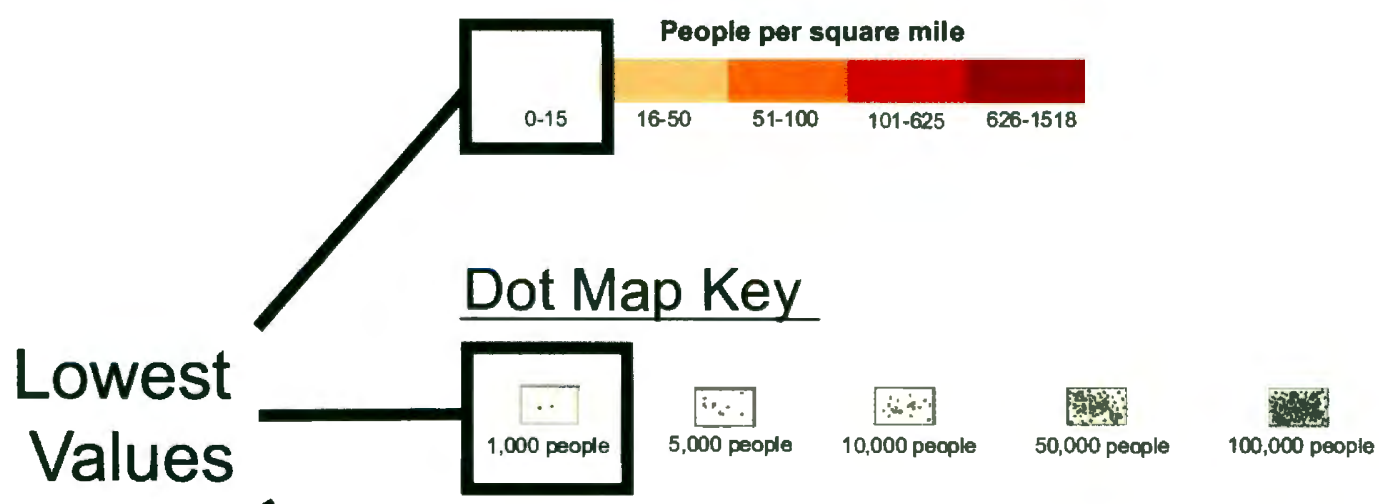

\section{Graduated Circle Map Key}

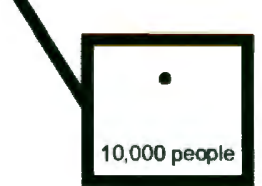

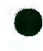

50,000 pecple

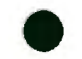

100,000 people

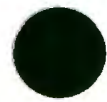

500,000 people

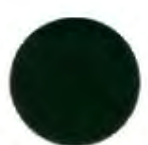

$1,000,000$ people

Figure 25. Lowest value in each map key. The key in the dot map shows 1,000 people as the lowest value (although the verbal description explains that "one dot $=500$ people") while the key in the graduated circle map shows 10,000 as the lowest value. Cartographically speaking, this does not mean that the lowest value found on the map is the lowest value in the key. In the choropleth map, $0-15$ people per square mile is the lowest value, but is also the answer because there are only five values on the map and all can be included in the key.

Table 16. The percentage of students within each group that wrote the low est value in their map's key as the answer to Question 1. In the dot and graduated circle maps, this is an incorrect answer. However, in the choropleth map it is correct because the choropleth map only has five values and all can be displayed in the key.

\begin{tabular}{|r|r|r|r|}
\multicolumn{1}{r|}{ Map Type } & \multicolumn{1}{c}{$\begin{array}{c}\text { Lowest value } \\
\text { in key }\end{array}$} & $\begin{array}{c}\text { \% of each group } \\
\text { that put lowest } \\
\text { value as answer }\end{array}$ & $\begin{array}{c}\text { Correct } \\
\text { Answer for } \\
\text { Question 1? }\end{array}$ \\
\hline \hline Dot & 1,000 & 36.2 & No \\
\hline Graduated & 10,000 & 56.9 & No \\
\hline Choropleth & $0-15$ people $/ \mathrm{mi}^{2}$ & 17.6 & Yes \\
\hline
\end{tabular}


value used on the map. Judging from the results, students might not understand that the map key is intended as a guide from which map-readers derive data values.

Nonetheless, we should be hesitant to assume these are only problems observed with children. A third explanation may be that adults may make these same mistakes. However, we cannot support this explanation with tested evidence, but instead offer this explanation as a direction for future research. Therefore, we should look at the positive side of these results and work to improve map keys so that map readers are aware of how to use them. The findings to Question 1 illustrate that students can locate a county on the map, identify that it contains a low number of people, and consult the key for an answer; showing that they do recognize that the key will help them "unlock" the map's data, but need help understanding how to interpret and apply the map key.

The second unusual finding from Question 1's statistical results is that students can extract values better than expected from dot maps. This should be alarming because traditionally it is more difficult for people to determine exact values from dot maps (Provin 1977). When there are countless dots coalescing into an area, it is impossible to judge the exact number of dots and therefore one must make a best guess. In the exercise used in this study, the county from which students extracted data values only contained three dots. A student can easily distinguish these three from one another and use simple math to derive the exact value. Therefore, this result probably cannot be generalized to explain student's 
ability to extract all data from dot maps. If we were to test if students could correctly identify a heavily populated area, we would probably see different results. Yet, although this finding may not be useful to determine if students can extract all values from dot maps, it does explain that those analyzing the dot map do understand how to extract data values from the symbols.

Although the high percentage of students to answer Question 3 allows us to support Hypothesis 1 that sixth-grade students can interpret all three map symbologies, it also requires us to be aware that children need additional practice with more advanced map reading skills, as illustrated by the low percentage of students able to correctly answer Question 1. This should not be a surprising or discouraging result because sixth grade is the grade level that begins the process of meeting the eighth-grade geography standards. Therefore, students have two more years before they reach eighth grade in which they will be tested on these skills. Identifying deficiencies that affect the early stages of learning will only allow for more improvement as the sixth-graders progress through middle school. Additionally, it may be an issue with map design and may indicate that cartographers should work on developing more intuitive map keys.

\section{Making Inferences: Question 2}

Making inferences from map data is the next step that bridges the ability to read data from a map and make educated hypotheses about it. This research tested if students could perform this task with Question 2 that asked them to identify a county that would most likely have a large city in it. The high 
percentages of students in each map group to correctly answer this question provides support for Hypothesis 2:

\section{Sixth-grade students can make inferences by analyzing information on graduated circle maps, dot maps, and choropleth maps.}

Additionally, answers for each map group did not deviate from expected counts, indicating that the three map types displayed data equally well for students to make inferences. Two trends appeared in the data for Question 2 that can be investigated further. First, students most frequently identified Multnomah County as the place that contains a large city (Table 17).

Table 17. Percentage of students who
marked Multnomah County for Question 2.
\begin{tabular}{rc} 
Map Type & Percentage \\
\hline Choropleth & 80.9 \\
\hline Dot & 72.5 \\
\hline Graduated & 80.0 \\
\hline
\end{tabular}

Because the percentages show this tendency was consistent for all three map types, we must ask why students chose Multnomah County. From the data collected, it is impossible to provide a definite answer. Students could be familiar with Oregon's geography and know that Portland is Oregon's largest city and is located in Multnomah County. Conversely, they could have marked Multnomah County as a direct result from studying the map. One way to identify if prior 
knowledge introduced bias into this test is to compare those students' answers who live near Portland and those who do not.

Although no subjects of this study lived in Portland city proper, five classes of students live in the Portland metropolitan area and may be more familiar with which county Portland resides than those living in Eugene. However, a Pearson's chi-square test revealed that students' location (Portland Metro Area or Eugene) had no association with whether they marked Multnomah County as the one that was most likely to contain a city, $\chi^{2}(1,202)=2.58, \mathrm{p}>.05$ (Table 18).

Table 18. Chi-square contingency table for location of students and those who marked Multnomah County for Question 2.

\begin{tabular}{|c|c|c|c|c|}
\hline Location & & $\begin{array}{r}\text { Marked } \\
\text { Multnomah }\end{array}$ & $\begin{array}{r}\text { Did Not } \\
\text { Mark } \\
\text { Multnomah }\end{array}$ & Total \\
\hline \multirow{4}{*}{$\begin{array}{c}\text { Portland Metro } \\
\text { Area }\end{array}$} & Expected Count & 27.6 & 96.4 & 78 \\
\hline & Observed Count & 23.0 & 101.0 & 78 \\
\hline & Residual & -4.6 & 4.6 & \\
\hline & Std. Residual & -0.9 & 0.5 & \\
\hline \multirow{5}{*}{ Eugene } & Expected Count & 17.4 & 60.6 & 124 \\
\hline & Observed Count & 22.0 & 56.0 & 124 \\
\hline & Residual & 4.6 & -4.6 & \\
\hline & Std. Residual & 1.1 & -0.6 & \\
\hline & Tota & 45.0 & 157.0 & 202 \\
\hline
\end{tabular}

I should note that this is not a fool-proof test because even those students who attended Eugene schools may be aware of Portland's location and mark Multnomah County as a result. Further investigation using a map that students are completely unfamiliar with is needed to be certain they chose Multnomah County as a result of analyzing the map data. This was advised against for this 
study because we felt students needed a map of a location they could identify themselves with so they felt more comfortable and invested in answering questions.

Although most students were able to provide the correct answer for Question 2, there were fourteen students who provided incorrect answers to Question 2. The second trend that appeared in the data was that half of those students who gave incorrect answers, or seven total, marked either Harney, Lake, or Lane Counties as the county most likely to have a city in it. This is worth noting because these three counties have three of the largest areas in Oregon. Their answer, then, may either be a result of chance or, alternatively, problems with what Piaget refers to as conservation (as described in Chapter 2). Piaget suggested that students who have not mastered conservation do not understand that an object's position or arrangement in space does not affect its physical properties (such as population). Therefore, these students may have difficulty understanding that because one county is larger than another does not mean that its population must also be larger.

Although further testing is needed to test this phenomenon, we should instead focus on the findings indicating that the three maps displayed data equally well from which students can make inferences. 


\section{Spatial Pattern Recognition: Question 4}

Another task that follows symbol interpretation is the ability to classify similar symbol values into groups. Question 4 requires students to draw regions around similar data values to indicate areas of highs, mediums, and lows.

Between 50 and 63 percent of each map group was able to identify regions and provides support for Hypothesis 3:

Sixth-grade students can identify and classify regions of similar values on graduated circle maps, dot maps, and choropleth maps.

Although the percentages are not overwhelmingly convincing, more than half of each group was able to classify regions. Additionally, many students who were considered unable to classify regions showed precursory steps to being able to do so. These findings are explained below. Of those students who were not counted as able to correctly draw regions, many of them labeled each county and drew no lines as illustrated in Figure 26. 

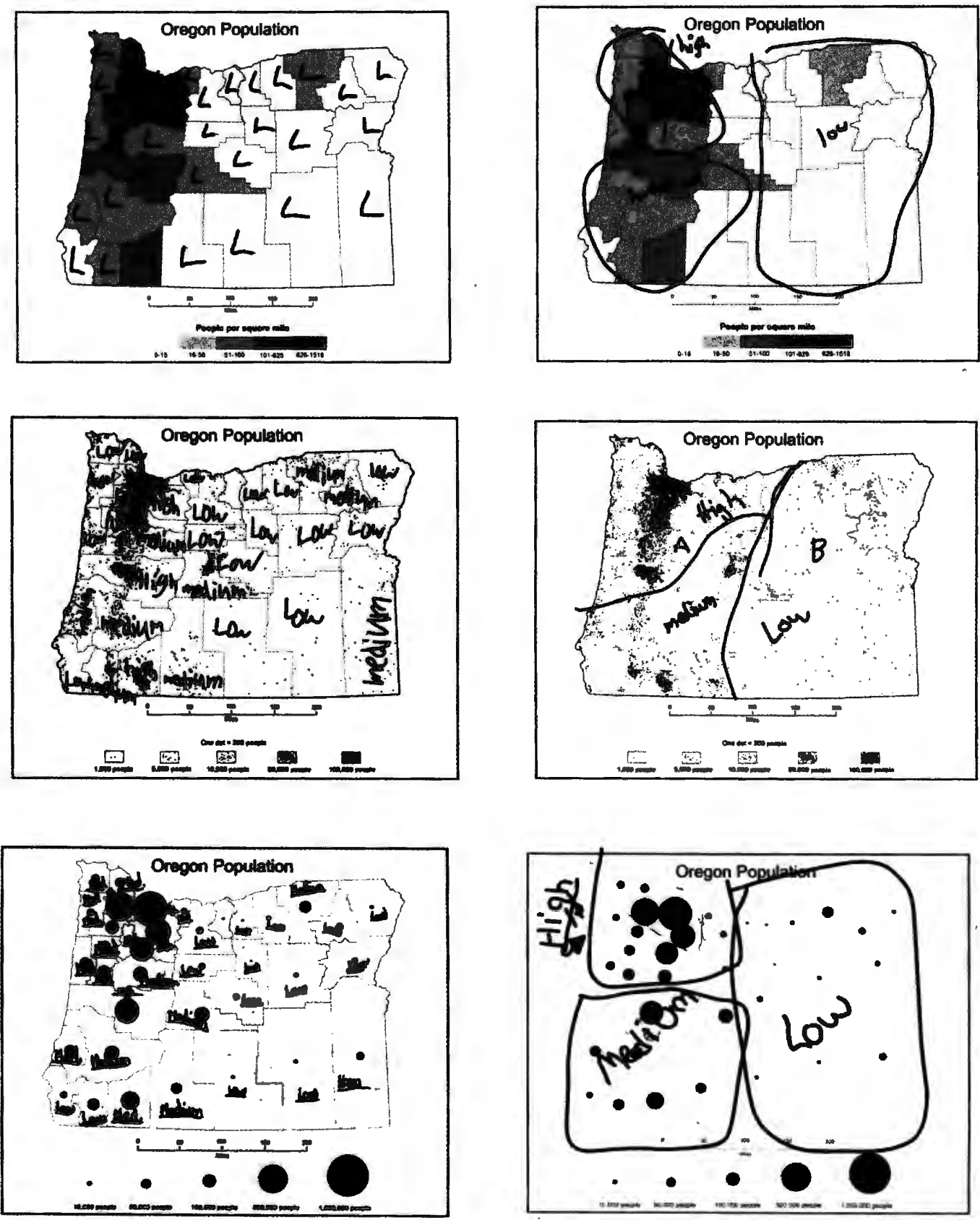

Figure 26. Examples of county labeling provided by students. The three examples in the left column illustrate the phenomena of labeling each county without drawing any regions, while the examples in the right column show proper region labeling. 
Figure 27 summarize these statistics and illustrate that a high percentage of students only labeled counties without identifying large regions. A Pearson's chiIncorrect answers that labeled just counties

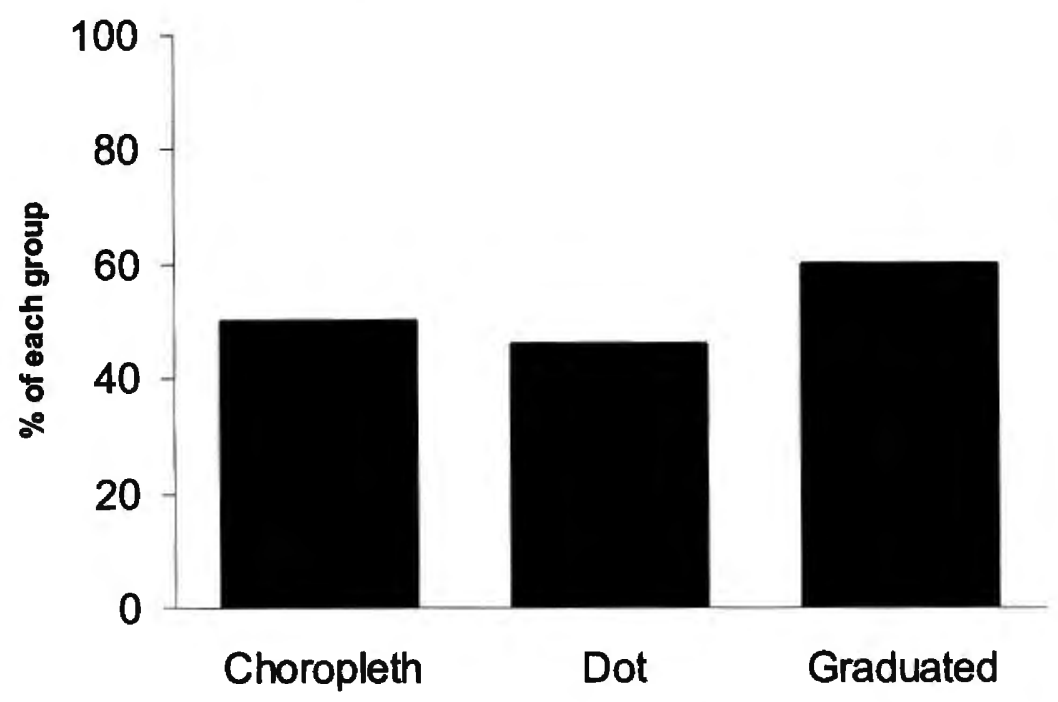

Figure 27. Percentage of incorrect answers that only had labeled counties.

square test of the students who simply labeled each county instead of drawing regions showed no differences than expected by chance, $\chi^{2}(2,83)=1.06, p>.05$ (Table 19). In other words, the chi-square test result indicates this was an occurrence consistent for all three maps and may reveal a few problems we should be aware of. Students may have misinterpreted the question and thought they had to label each county. This may be caused by the last sentence in the question that instructs students that, "When you get done, every place in Oregon should be in a high, medium, or low region." 
Table 19. Chi-square contingency table for those of the incorrect answers labeled each county.

\begin{tabular}{clrrr} 
Map Type & & & Only & \\
\hline \multirow{4}{*}{ Choropleth } & Incorrect & Labels & Total \\
& Expected Count & 13.2 & 12.8 & 26 \\
& Observed Count & 14.0 & 12.0 & 26 \\
& Residual & 8.3 & -8.3 & \\
\cline { 2 - 5 } & Std. Residual & $\mathbf{0 . 2}$ & $-\mathbf{0 . 2}$ & \\
\hline \multirow{4}{*}{ Dot } & Expected Count & 12.7 & 12.3 & 25 \\
& Observed Count & 14.0 & 11.0 & 25 \\
& Residual & -15.3 & 15.3 & \\
\cline { 2 - 6 } & Std. Residual & 0.4 & -0.4 & \\
\hline \multirow{3}{*}{ Graduated Circle } & Expected Count & 16.2 & 15.8 & 32 \\
& Observed Count & 14.0 & 18.0 & 32 \\
& Residual & 7.0 & -7.0 & \\
\cline { 2 - 6 } & Std. Residual & -0.5 & 0.6 & \\
\hline
\end{tabular}

Another explanation could be that students were distracted from identifing regions because they were focused on interpreting each symbol. If this was the case, then this might be the precursory step to identifying regions similarly to what Lange-Küttner (2006) discovered. As described in Chapter 2, Lange-Küttner found that younger children have difficulties creating a plan to scan an entire page of data in order to classify symbols into groups. Instead, they get distracted by interpreting symbols and this affects the way they classify. If students practice more and develop their spatial cognitive skills, the next logical step would be to classify those individual values into regions.

Over half of each map group was able to delineate regions. Of those students who were able to identify regions, two notable trends appeared. First, students either drew boundaries around symbols or they drew boundaries directly 
following county boundaries as illustrated in Figure 28. This was an occurrence common to all three map types (Figure 29).

Regions Follow Boundaries
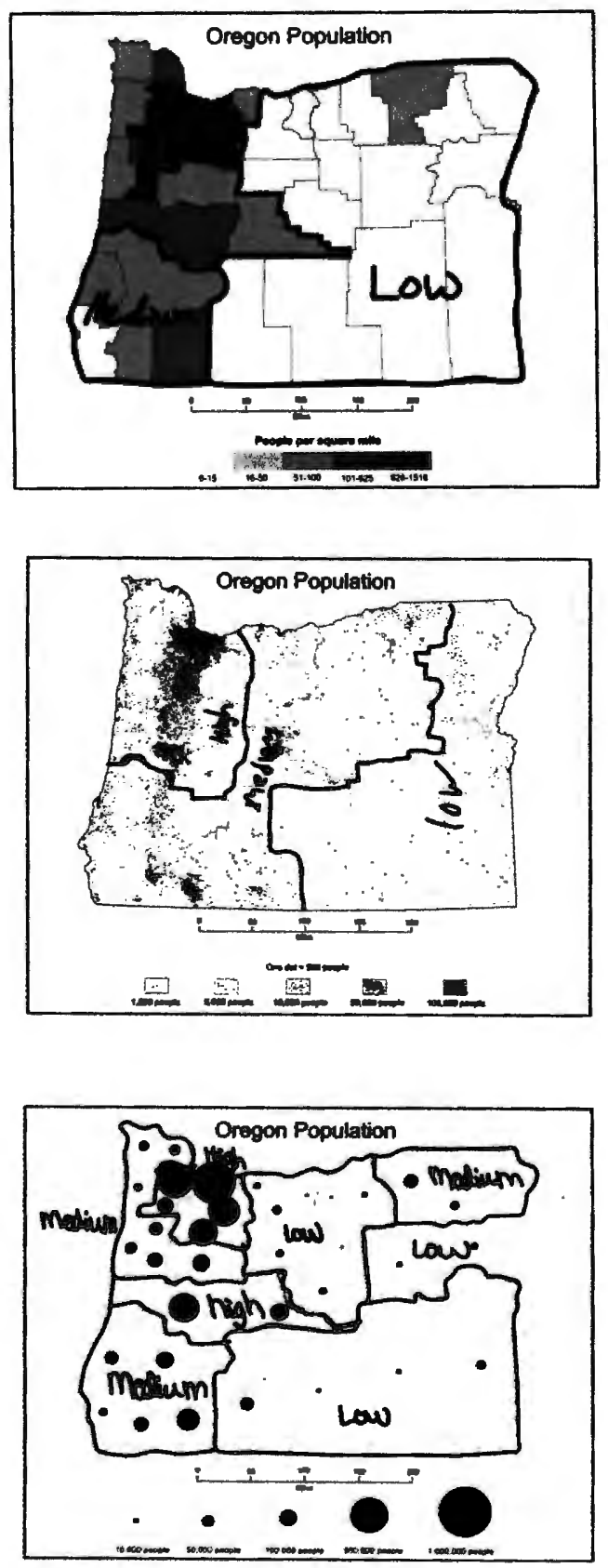

Regions Follow Symbols
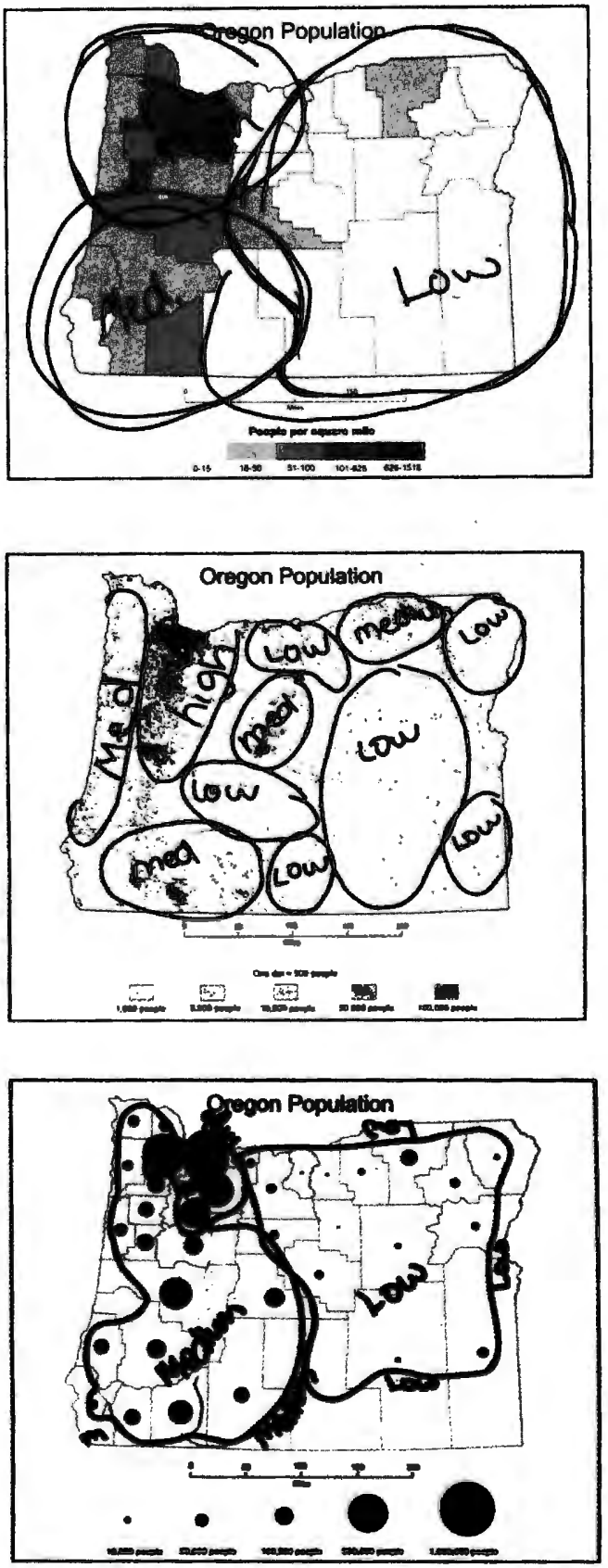

Figare 28. Examples from students illustrating region lines following county boundaries. 
Correct answers that followed county boundaries

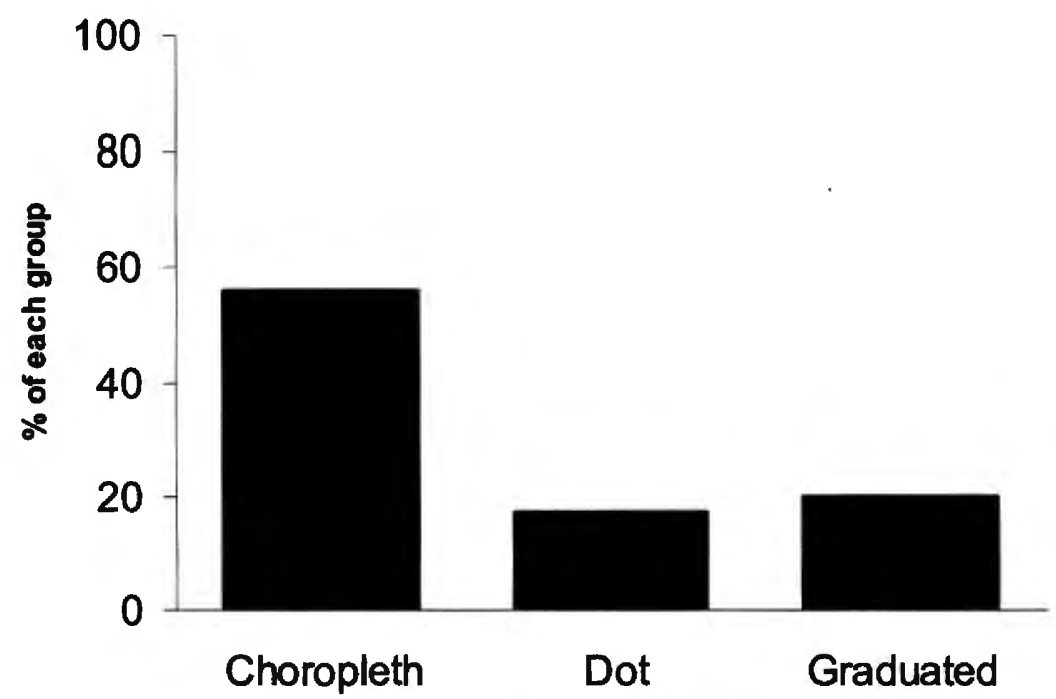

Figure 29. Percentage of correct answers that followed county boundaries.

\begin{abstract}
Although some students in all map groups used county boundaries as the division line, those analyzing the choropleth map did so at a much higher rate. A statistically significant Pearson's chi-square test reveals that the number of students who drew lines directly on county borders exceeded what was expected by chance, $\chi^{2}(2,119)=36.19, p<.05$ (Table 20$)$. The standardized residuals indicate that students more often drew lines/circles directly on the county borders of the choropleth map regions and drew lines/circles directly on county borders of the dot map less frequently than expected.
\end{abstract}


Table 20. Chi-square contingency table for region boundaries to follow county borders.

\begin{tabular}{|c|c|c|c|c|}
\hline Map Type & & $\begin{array}{r}\text { Did not } \\
\text { draw on } \\
\text { boundaries }\end{array}$ & $\begin{array}{r}\text { Drew on } \\
\text { county } \\
\text { boundaries }\end{array}$ & Total \\
\hline \multirow{4}{*}{ Choropleth } & Expected Count & 22.2 & 19.8 & 42 \\
\hline & Observed Count & 7.0 & 35.0 & 42 \\
\hline & Residual & 8.3 & -8.3 & \\
\hline & Std. Residual & -3.2 & 3.4 & \\
\hline \multirow{4}{*}{ Dot } & Expected Count & 23.3 & 20.7 & 44 \\
\hline & Observed Count & 35.0 & 9.0 & 44 \\
\hline & Residual & -15.3 & 15.3 & \\
\hline & Std. Residual & 2.4 & -2.6 & \\
\hline \multirow{4}{*}{ Graduated Circle } & Expected Count & 17.5 & 15.5 & 33 \\
\hline & Observed Count & 21.0 & 12.0 & 33 \\
\hline & Residual & 7.0 & -7.0 & \\
\hline & Std. Residual & 0.8 & -0.9 & \\
\hline & Tota & 63.0 & 56.0 & 119.0 \\
\hline
\end{tabular}

This could be explained by a few possibilities. First, the beginning questions in the survey ask about counties (i.e. which county has a higher population, which county probably has a city, etc.). We could have "primed" students to think in terms of county boundaries. Another logical explanation is the inherent properties of the symbols. In a choropleth map, the entire county is shaded a uniform color and the county border serves as the edge of the symbol. The dot and graduated circle maps leave spaces on the map between the symbols that allows students to make decisions about where to draw the line: on the county border or around the symbols.

If this phenomenon is not a result of priming students to think within county boundaries, it might suggest that divisions such as county boundaries provide logical breaks for students, which may or may not be conducive to 
geographic learning. In some cases, it may be important to identify phenomena by county, but in most cases it is important to recognize geographical distributions beyond arbitrary political boundaries. Cartographers should be aware of this finding that children group based on county boundaries because cartographers frequently include political boundaries for reference information, especially in children's literature.

The second pattern visible in the data was the number of regions students drew as summarized in Table 21. Because Question 4 allowed students to draw as

Table 21. Summary statistics for number of regions drawn.

\begin{tabular}{rrrrrrr} 
Map Type & $\begin{array}{c}\text { Mean \# of } \\
\text { regions }\end{array}$ & \multicolumn{1}{c}{ Median } & Minimum & Maximum & $\begin{array}{l}\text { Standard } \\
\text { Deviation }\end{array}$ \\
\hline Choropleth & 5.78 & 3 & 3 & 36 & 6.47 \\
\hline Dot & 6.30 & 3 & 3 & 36 & 7.96 \\
\hline Graduated & 7.03 & 3 & 3 & 36 & 8.99 \\
\hline
\end{tabular}

many regions as they deemed appropriate, there were multiple correct responses that were accepted as long as they correctly labeled the regions. The graduated circle map has both the highest mean number of regions and the largest standard deviation, the dot map second, and the choropleth the least number of regions. However, a one-way ANOVA test on the number of regions drawn between regions produced no statistically significant results, $F(2,199)=1.01, p>.05$. One might expect that the dot map would have the highest number of regions because it has essentially an infinite amount of "symbol values." In other words, it 
graphically presents the strongest image of "numerousness" because map readers are required to count many discrete symbols. Because the visual task of the dot map is to identify density values of the data, there are an infinite number of regions of different densities that can be identified on the map.

Following this logic, one might expect the graduated circle map to have the second highest number of regions because it has the next largest amount of symbol values. In the graduated circle map, each circle is a different size because each county population is different. This results in 36 different symbol sizes.

Lastly, the choropleth map only provides five symbol values because it classes data into groups. Therefore, we might expect students to see fewer regions on the choropleth map. Yet, the ANOVA test reveals that the groups were not statistically different. Thus, we can conclude the maps presented data equally well for students to identify regions of similar values.

To summarize, more than half of each group was able to correctly interpret symbology and categorize similar values into regions. Also, many students showed precursory steps to being able to draw regions. Those students analyzing the choropleth map drew region boundaries directly on county borders at a higher frequency than expected compared to the dot and graduated circle maps. Nevertheless the three map types presented data equally well for this task. These findings provide support for Hypothesis 3 that students are able to class similar values into regions on choropleth, graduated circle, and dot maps. 


\section{Thinking Geographically: Question 5}

One skill that illustrates that a student has understood the data is the ability to take that comprehended data and inquire why or how the data patterns exist. Question 5 illustrates students have developed skills essential to geography's methodologies and the results support Hypothesis 4:

\section{Viewing symbologies on these maps can help students formulate geographic questions.}

Between 69 and 82 percent of each map group was able to provide at least one geographic question that inquired about data patterns, supporting Hypothesis 4 that sixth-graders can generate geographic questions by viewing map symbologies. Additionally, all maps presented data equally well for this task as determined by an insignificant chi-square test result.

The ability to ask questions about the data derives from the ability to identify distributions and patterns on maps. It would seem logical that questions about patterns and distributions would be more easily obtained from dot maps because dot maps most closely resemble the actual patterns and distributions of the data. However, students' performance on asking a geographic question about the data did not statistically deviate from performance expected by chance, suggesting all maps displayed data equally well for this task. Although this finding strays from what we might expect, it is encouraging for educators because 
it illustrates that all three maps are equally useful for showing distributions that will lead to further inquiry of the data.

Additionally, the types of questions students asked were similar no matter the type of map students read (Table 22). This was verified by a Pearson's chi-

Table 22. Types of correct questions asked. Most students asked about why the western part of the state was more populated than the eastern (West vs. East). However, others asked whether physical geography such as weather or landforms explains why people live where they do (Physical geography), why there is a trend for counties with small areas to have the largest populations (Many in small places), or about whether population has always remained this way (Movement/change).

\begin{tabular}{r|rrrrr}
\hline \multicolumn{1}{c}{ Map Type } & West vs. East & $\begin{array}{c}\text { Physical } \\
\text { geography }\end{array}$ & $\begin{array}{c}\text { Many in small } \\
\text { places }\end{array}$ & $\begin{array}{c}\text { Movement } \\
\text { /change }\end{array}$ \\
\hline Choropleth & 40 & 8 & 5 & 3 \\
\hline Dot & 32 & 5 & 2 & 1 \\
\hline Graduated & 25 & 4 & 13 & 1 \\
\hline
\end{tabular}

square test that produced no statistically significant results. The most frequent question in the data was something similar to, "Why is the western part of the state more populated than the eastern side?"

An unexpected pattern was obtained from this question that does not directly correspond to the hypotheses, but is important to note as evidence of map learning. Eighty-eight students used cardinal directions in their questions, although the map did not include a directional arrow. Out of the 88 who used a directional comparison, only four of them did not use cardinal direction names and instead used phrases such as "upper left" or "bottom right." Additionally, everyone who used a cardinal direction, did so correctly; illustrating that by sixth grade, many students are skilled in orientation. This finding may be a result of 
early exposure to and practice with general reference maps in previous educational exercises.

The results obtained from Question 5 allow us to conclude that all three symbologies display data effectively so that students can formulate geographic questions equally well for each map. Yet, there is still more room for improvement as students progress through their middle school curriculum. Teaching students how to identify patterns on maps and inquire about the patterns' existence is a crucial step to having them become well-rounded geographic thinkers. Fortunately, many sixth-graders seem to be well on their way to being competent at this task as illustrated with the results from Question 5.

\section{Understanding the Symbolization: Question 6}

The results of Question 6 that asks students to explain their map's symbolization suggest that all maps were equally challenging to students. However, because this was a question intended to help clarify other answers, it was not directly counted as identifying if a student could interpret, infer, classify, and inquire about the map data. Additionally, I would be surprised to find that adults could perform better on this map-reading task because the ability to explain in one's own words how the map works is difficult. Taking that into consideration, educators should be encouraged that at least a third of students in each map group can explain their map's symbology. 


\section{Overall Performance}

Students' overall performance, as measured by the summation of correct answers of Questions 1 through 5, allows us to conclude that students can understand the three thematic maps. Statistically speaking, students perform better on dot maps and worse on graduated circle maps than expected by chance. This indicates that the dot map is the most effective, but this result should be evaluated cautiously. Nearly all students were able to correctly answer more than half of the five questions, no matter which map type they analyzed. This indicates that all three maps can be used in classroom skill instruction, but it might suggest that if one were to give a presentation to sixth-graders, one might use a dot map to display data because more students would understand more aspects of it as a whole than one of the other two maps. However, it does not mean that the other two maps should be taken from the curriculum, because students do understand them. Only two students were not able to correctly answer any questions about the map. Although students performed best on the dot map, the other two map types still worked and students were able to read and interpret them, making all three map types viable as communication devices.

\section{Student Demographic Characteristics}

Students' overall performance and student characteristics (i.e. class, teacher, age, gender) have no associations as determined by an insignificant chisquare test, allowing us to conclude that students' overall performance is 
independent of these four traits. Of greatest concern to most researchers is gender. This study revealed no difference in performance between girls and boys. This may be a result of today's society providing similar situations to both genders than societies may have in the past.

Secondly, a student's performance was independent of his or her teacher or class. This result allows us to conclude that different methods of instruction and classroom skill-level are not associated with students' abilities to read these quantitative thematic maps. More importantly, it allows us to understand that sixth-graders have been influenced by many teachers and other educational experiences inside and outside the classroom. Thirdly, there was no association between age and performance. However, most students in sixth-grade are 11 and 12 years old and Piaget would predict that at the same age students would be at similar levels of development. Because overall performance had no association with gender, teacher, class, or age, we can more easily generalize our findings to the broad population of Oregon sixth-graders.

\section{Summary}

Overall, students performed better than expected at analyzing dot maps than choropleth or graduated circle maps. Although this finding is important, the performance of each task may be more useful for educators to design curriculum or cartographers to improve map design for children. The summary of the four tasks' performances are outlined in Table 23. It illustrates that students can 
understand these three thematic maps and use them to advance their geographic skills.

Table 23. Hypothesis overview.

\begin{tabular}{|c|c|c|c|}
\hline Hypothesis & $\begin{array}{l}\text { Was the } \\
\text { Hypothesis } \\
\text { Supported? }\end{array}$ & What Evidence? & $\begin{array}{c}\text { Any performance } \\
\text { deviances by } \\
\text { map type? }\end{array}$ \\
\hline $\begin{array}{l}\text { \#1: Interpret } \\
\text { symbology }\end{array}$ & Yes & $\begin{array}{l}90 \% \text { of students } \\
\text { answered Question } 3 \\
\text { correctly. However, } \\
\text { between } 3 \text { and } 35 \% \text { of } \\
\text { each group answered } \\
\text { Question } 1 \text { correctly, } \\
\text { showing that students } \\
\text { do need more practice } \\
\text { with this task. }\end{array}$ & $\begin{array}{l}\text { On Question } 3 \text {, no. } \\
\text { On Question } 1 \text {, yes. } \\
\text { Dot map performance } \\
\text { was better than } \\
\text { expected and } \\
\text { graduated circle was } \\
\text { worse than expected }\end{array}$ \\
\hline $\begin{array}{l}\text { \#2: Make } \\
\text { Inferences }\end{array}$ & Yes & $\begin{array}{l}\text { Between } 90 \% \text { and } 95 \% \\
\text { of each group was able } \\
\text { to answer Question } 2 \\
\text { correctly. }\end{array}$ & No \\
\hline \#3: Draw Regions & Yes & $\begin{array}{l}\text { Between } 50 \% \text { and } 63 \% \\
\text { of each group correctly } \\
\text { drew regions as } \\
\text { required in Question 4, } \\
\text { while others showed } \\
\text { precursory steps to } \\
\text { correctly perform this } \\
\text { task. }\end{array}$ & No \\
\hline \#4: Ask Questions & Yes & $\begin{array}{l}\text { Between } 70 \% \text { and } 82 \% \\
\text { of each group was able } \\
\text { to ask a geographic } \\
\text { question about the map } \\
\text { data }\end{array}$ & No \\
\hline
\end{tabular}




\section{RECOMMENDATIONS AND CONCLUSIONS}

The findings of this study support the four hypotheses that show students can understand quantitative thematic maps. Results support Hypothesis 1 that students can interpret symbology from the three maps. Nearly $90 \%$ of students in each map group were able to interpret their map's symbology to determine which of two counties had the higher population. However, a low percentage of each map group was able to correctly extract data values from one county's symbolization, with dot maps statistically being easier for students. The results of these two tasks together suggest that sixth-graders can interpret map symbology, but need additional practice understanding higher-order tasks of extracting exact values, which may or may not be a problem unique to sixth-graders.

Nevertheless, teachers should recognize this deficiency and develop strategies to aid students with this task. To do this, teachers could begin teaching students how to extract data from dot maps because students obtained data values more frequently with this type of map. Following Vygotsky's theory of scaffolding, once students understand how to extract data from dot maps, the choropleth and graduated circle maps' symbology could be compared and contrasted to the dot map's in order to help students understand how to read data values from these more difficult maps. This strategy might improve students' abilities to extract exact data values from maps. 
The finding that nearly three quarters of students in each map group were able to identify a county that probably has a city in it supports Hypothesis 2 that students can make inferences from the three maps. The results indicate that there is still room for improvement on this map reading task, especially if students were able to answer this question as a result of being familiar with Oregon's population. With this understood, educators can slowly guide students to be proficient at this task. First, an Oregon teacher could provide his or her students with a population map of Oregon because students can identify with the state in which they live. Then, the teacher could emphasize how Portland is located in Multnomah County and how the county's symbology explains why a city might be located there. Next, the teacher could provide another map that depicts an unfamiliar region to the students. The teacher could point out similarities of this map with the Oregon map and then allow students to infer from the data on the unfamiliar map. Again, employing Vygotsky's theory of scaffolding, students should gradually improve their abilities to make inferences in addition to gaining a more comprehensive understanding of the maps' symbologies and distributions they present.

The finding that over $50 \%$ of students in each map group were able to correctly draw regions, with more showing precursory steps to being able to identify regions, supports Hypothesis 3. Students' performance on this task showed no deviations from what is expected, suggesting the three map symbologies displayed data equally well for allowing students to categorize 
similar values into regions. Educators can direct students in specific areas to improve students' abilities to complete this task. For example, if the students' inability to draw regions was caused by the inability to understand the symbols instead of the way the question was phrased (as discussed in Chapter 5), then teachers may want to follow advice from Lange-Küttner (2006) for this task. This would require teachers to instruct students to study the overall map before they focus on one symbol. Additionally, teachers may demonstrate how to draw regions to illustrate this skill and then instruct students to work on their own with other maps. Nevertheless, students are at the beginning stages of using these thematic maps to identify regions.

The finding that three quarters of each map group was able to generate a geographic question supports Hypothesis 4. Performance on this task did not deviate from what is expected by chance, suggesting each map was equally effective at displaying information to allow students to inquire about certain data patterns. This should be encouraging to geographers because this shows that students not only understand different map symbologies, but can use them all equally well to identify patterns and question distributions, illustrating that they are developing skills essential to the discipline of geography.

The results from all five tasks, in addition to the overall combined score results, indicate that sixth-grade students do understand quantitative thematic maps. Therefore, we can argue that these maps should be used more frequently in school curriculum to help develop students' map skills to ensure they develop 
spatial cognitive abilities essential to geography. Additionally, if sixth-grade students practice with these types of maps, by the time they reach eighth grade where they will be tested on these abilities to meet state standards, they will be proficient at using quantitative thematic maps.

Overall, these results yield certain recommendations for educators and research opportunities for cartographers. One recommendation to curriculum planners and educators is to integrate map work into other subjects such as math. In schools, there are many subjects that need to be taught, each competing for precious classroom time. Therefore, only the most important concepts and skills are taught within each discipline. If we combine concepts of certain subjects, we save time and therefore students can be exposed to more content. This would work well with maps. For example, students will be taught fractions and other complex computations in math, which could be paired with map work. Students could compute population densities for each county in Oregon. This would allow them to practice multiplication and division skills. Then, they could classify the data values into groups to determine the symbol to apply to each county. This will introduce the concept of mathematical ranges and illustrate different methods to measure central tendency. Using such exercises will provide three benefits. First, it will supply students with real-world data to work with which makes learning the mathematical methods more applicable. Secondly, it will apply math skills to a graphic that will help visual learners understand the often-difficult 
mathematical concepts. Third, it will allow teachers to introduce more quantitative maps for practice.

Another recommendation to educators is to instruct students how thematic maps are actually made by cartographers or have students make maps themselves as a means to learn what is required to produce specific types of maps. After the students took the survey, I explained to them how each map showed the same data, but used different symbology to do so. I then highlighted the strengths and weaknesses of each map, to which students were completely intrigued. Once they realized each map had negative attributes to it, they began to raise their hands to offer suggestions on how a cartographer could improve the design for more effective communication. This enthusiasm displayed in each class suggests that once students understand how a map is created, they seem more likely to understand how to read it. Thus, it might be a useful strategy for teachers to employ mapmaking as a way for children to understand maps.

Cartographers can also aid students by working to improve the clarity of specific map elements. This study indicates that students' performance may be affected by map key misunderstanding, so a recommendation to cartographers is to design alternative map keys for children. By using map keys such as the three presented in Figure 30, cartographers may be able to provide a missing link between replicative symbols and abstract symbols. The three map keys also take into consideration that students had problems understanding that map keys on dot 
GRAPHICAL KEY FOR DOT MAP

$$
\begin{gathered}
-\quad=\quad \ell^{\ell} ! \\
\text { one dot } \quad \text { five people }
\end{gathered}
$$

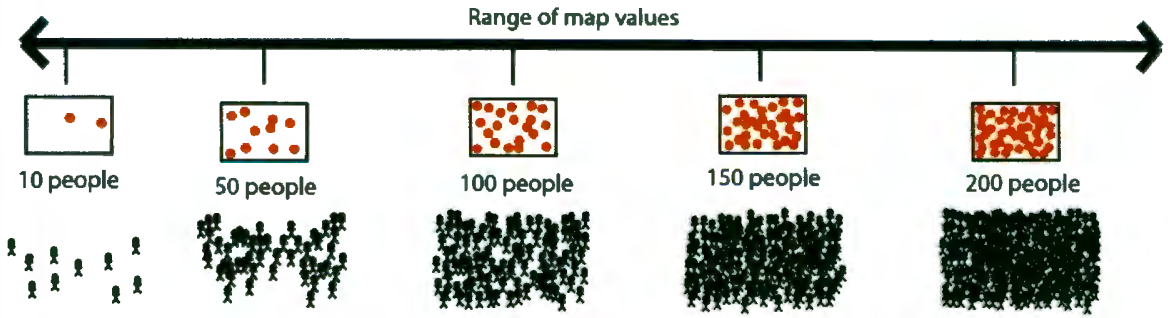

As the dots get closer together, the more people live in one space.

These are five examples.

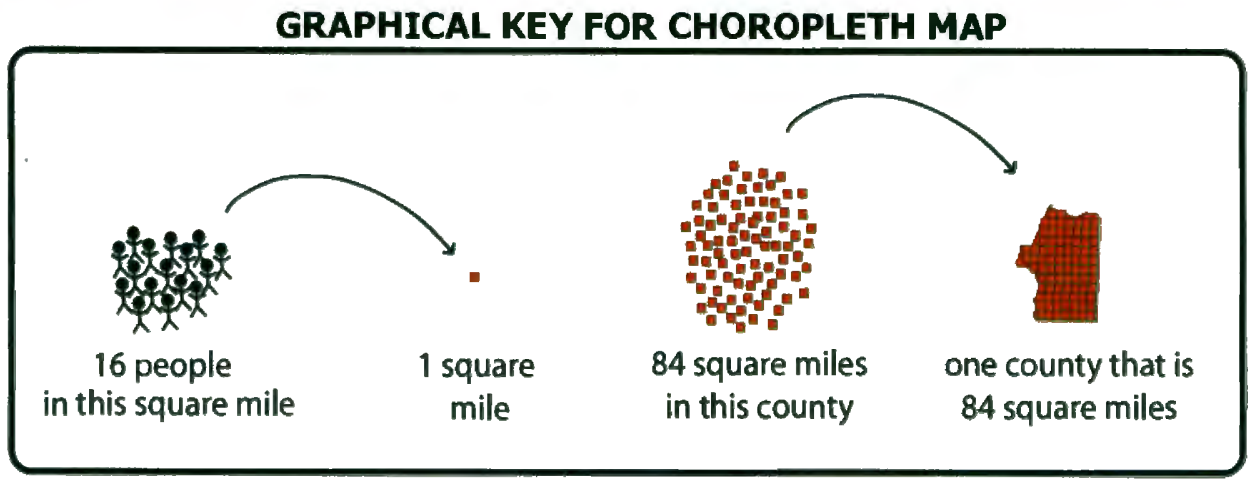

GRAPHICAL KEY FOR GRADUATED CIRCLE MAP

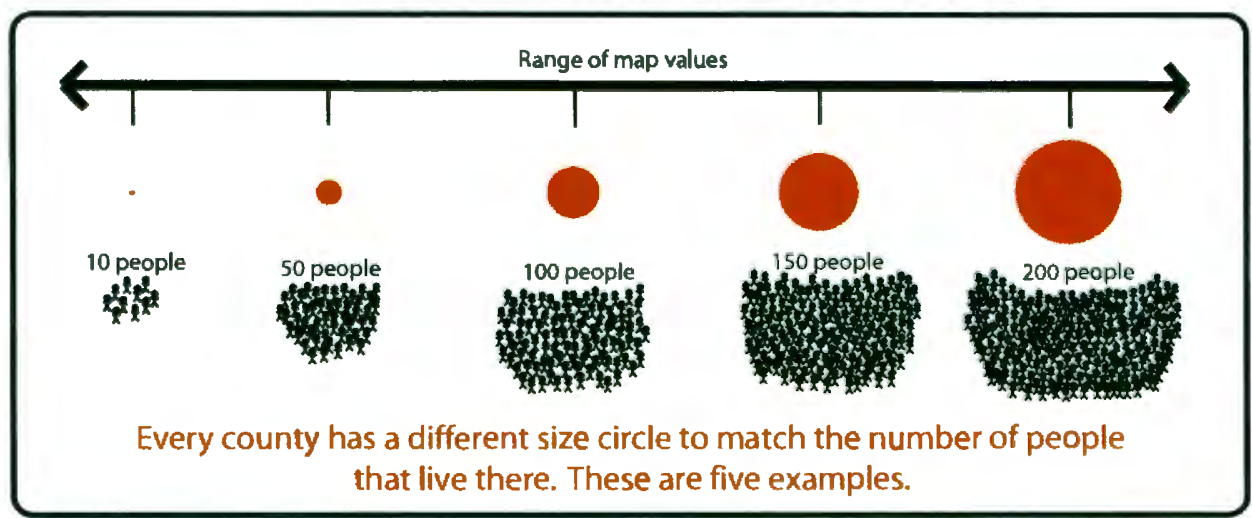

Figure 30. Examples of supplemental map keys. All three use pictorial symbols in coordination with the more abstract symbols used on the map. The dot map key attempts to illustrate the concept of density while showing that the five values presented in the key are five from many found in the "range of map values" bar. The choropleth map key attempts to illustrate the meaning of "people per square mile" through a simple example. The graduated circle key attempts to illustrate that the size of the circle is directly proportional to the number of people present and that the five values are only five of many actually found on the map. 
and graduated circle maps depict only five values from a continuous range and that choropleth map keys display population density. Instead of providing the traditional map keys given to students in the survey, we could test the effectiveness of alternative designs such as those presented in Figure 30. Although these suggested map keys in Figure 30 may not depict the intended ideas most effectively, they offer one alternative option for each map that cartographers can test. Additionally, they illustrate that there are different approaches to making map keys, leaving an uncharted territory for cartographic researchers to explore.

Lastly, because middle school students can understand thematic maps, more specific standards should be written to ensure children are exposed to numerous types of maps. Perhaps before we pursue this endeavor, researchers might test if children are able to grasp geographic concepts such as regional patterns better if they simultaneously viewed more than one type of map displaying the same data. I would suspect children could compare and contrast how data is shown on the multiple maps to gain a more comprehensive understanding of the data. If this is found to be true, then it might provide a stronger argument to advocate that geography standards include a requirement for students to use a number of specifically identified quantitative thematic maps.

Nevertheless, the results obtained from this study suggest that the reason quantitative thematic maps are underrepresented in textbooks is not because students do not understand them. If this is the case, we can pose the question, "If students understand thematic maps, why aren't they used more frequently in 
school curriculum?" Students can use common quantitative thematic maps, specifically choropleth, dot, and graduated circle maps, to practice geographic skills such as pattern recognition, region formation, and geographic questioning. In addition, when they do use these quantitative thematic maps, they practice with numerical spatial data - data of greater potential information content than found on qualitative thematic maps. If we begin to trust that students do understand these quantitative thematic maps and can use them to refine their geographic skills, we will have them on the road to becoming inquisitive geographers. 


\section{WORKS CITED}

Ault, R. 1983. Children's cognitive development. New York: Oxford University Press.

Blades, M., S. Sowden, and C. Spenser. 1995. Young children's use of spatial relationships in tasks with maps and models. Cartographica, 32: 18-28.

Blaut, J.M., D. Stea, C. Spencer, and M. Blades. 2003. Mapping as a cultural and cognitive universal. Annals of the Association of American Geographers, 93:165-185.

Bluestein, M. and L. Acredolo. 1979. Developmental changes in map reading skill. Child Development, 50: 691-697.

Brewer, C. 1994. Color use guidelines for mapping and visualization. In Visualization and Modern Cartography, (Ed.) A.M. MacEachren and D.R.F. Taylor: 123-127. New York: Elsevier.

Castner, H.W. 1997. Modifying our view of geography in light of research in spatial development. In The first assessment: Research in geographic education, (Ed.) R.G. Boehm and J.F. Petersen: 46-53. San Marcos: The Gilbert M. Grosvenor Center for Geographic Education, Southwest Texas State University.

Chang, K. 1980. Circle size judgment and map design. American Cartographer, 7: 155-162.

Color Brewer. 2002. Sequential 5-Class Legend. Available at: http://www.personal.psu.edu/cab38/ColorBrewer/ColorBrewer.htm1. (Last accessed 10 October 2006).

DeLoache, J.S., Miller, K.F. \& Pierroutsakos, S.L. 1998. Reasoning and problem solving. In Handbook of Child Psychology: Vol 2. Cognition, perception, \& language_( $5^{\text {th }}$ ed.), (Eds.) D. Kuhn \& R. Siegler: $810-850$. New York: Wiley.

Dent, B.D. 1996. Cartography: Thematic Map Design ( $5^{\text {th }}$ ed.). Boston: McGrawHill.

Downs, R.M., L.S. Liben, and D. G. Daggs. 1988. On education and geographers: The role of cognitive developmental theory in geographic education. Annals of the Association of American Geographers, 78: 680-700.

Downs, R.M. and L.S. Liben. 1991. The development of expertise in geography: A cognitive-developmental approach to geographic education. Annals of the Association of American Geographers, 81: 304-327.

Flannery, J.J. 1971. The relative effectiveness of some common graduated point symbols in the presentation of quantitative data. The Canadian Cartographer, 8: 96-109.

Geography Education Standards Project (GESP). 1994. Geography for Life: National Geography Standards 1994. Washington, DC: National Geographic Research and Exploration. 
Gerber, R. 1984. The development of competence and performance in cartographic language by children at the concrete level of map-reasoning. Cartographica, 21: 99-119.

Gregg, M. 1997. Problem posing from maps: Utilizing understanding. Journal of Geography, 96: 250-256.

Griffin, T.L.C. 1985. Group and individual variations in judgment and their relevance to the scaling of graduated circles. Cartographica, 22: 21-37.

Griffin, T.L.C. 1990. The importance of visual contrast for graduated circles. Cartography, 19: 21-30.

Gilmartin, P.P. 1981. Influences of map context on circle perception. Annals of the Association of American Geographers, 71: 253-258.

Hetherington, E.M., Parke, R.D., Gauvain, M., Locke, V.O. 2006. Child Psychology: A contemporary Viewpoint. Boston: McGraw-Hill.

Jenks, G.F. 1974. The average map-reader lives! Paper presented at annual meeting of the Association of American Geographers.

Kotovsky, L. and Gentner, D. 1996. Comparison and categorization in the development of relational similarity. Child Development, 67: 2797-2822.

Lange-Kuttner, C. 2006. Drawing boundaries: From individual to common region- the development of spatial region attribution in children. British Joumal of Developmental Psychology, 24: 419-427.

Liben, L.S. and R.M. Downs. 1994. Fostering geographic literacy from early childhood: The contributions of interdisciplinary research. Joumal of Applied Developmental Psychology, 15, 549-569.

Leinhaardt, G., C. Staintoon, J.M. Bausmith. 1998. Constructing maps collaboratively. Journal of Geography, 97: 19-30.

MacEachren, A.M. 1995. How Maps Work: Representation, Visualization, and Design. New York: Guilford Press.

Mackay, J.R. 1949. Dotting the map: An analysis of dot size, number and visual tone density. Surveying and Mapping, 9:3-10.

Mandler, J.M., P.J. Bauer, and L. McDonough. 1991. Separating the sheep from the goats: Differentiating global categories. Cognitive Psychology, 23: 263298.

Marzolf, D.P. and DeLaoche, J.S. 1994. Transfer in young children's understanding of spatial representations. Child Development, 65: 1-15.

Mashoka, Z., H.H.L. Bloemer, and J. Pickles. 1986. Dot maps vs. proportional circle maps, an assessment of readability, legibility and preference. The Society of University Cartographers Bulletin, 20: 1-6.

McGrew, J.C. and C.B. Monroe. 2000. An Introduction to Statistical Problem Solving in Geography ( $\left.2^{\text {nd }} e d\right)$. Boston: McGraw Hill.

Michaelidou, E.C., B.P. Nakos, and V.P. Filippakopoulou. 2004. The ability of elementary school children to analyze general reference and thematic maps. Cartographica, 39: 65-88.

Newcombe, N.S. and J. Huttenlocher. 2000. Making Space: The Development of Spatial Representation and Reasoning. Cambridge: MIT Press. 
Oregon Department of Education (ODE). 2006. Social Studies Standards in Geography. Available at :

http://www.ode.state.or.us./teachlearn/real/standards/. (last accessed 2 October 2006).

O'Sullivan, D. and D. J. Unwin. 2003. Geographic Information Analysis. New York: John Wiley \& Sons.

Piaget, J. 1970. Piaget's theory. In Carmichael's Manual of Child Psychology, (Ed.) Ph.H. Mussen, New York: John Wiley \& Sons.

Provin, R.W. 1977. The perception of numerousness on dot maps. The American Cartographer, 4: 111-125.

Quin, P.C. 2002. Early categorization: A new synthesis. In Blackwell Handbook of Childhood Cognitive Development, (Ed.) U. Goswami: 84-101. Oxford: Blackwell Publishing.

Rittschof, K. A. and R.W. Kulhavy. 1996. Learning and remembering from thematic maps of familiar regions. Educational Technology Research and Development, 46: 19-38.

Robinson, A. H., R.D. Sale, J.L Morrison, and P.C. Muehrcke. 1984. Elements of Cartography $\left(5^{\text {th }} \mathrm{ed}\right.$.). New York: John Wiley \& Sons.

Rossano, M.J. and T.T. Morrison. 1996. Learning from maps: General processes and map-structure influences. Cognition and Instruction, 14: 109-137.

Sadahiro, Y. 2000. Perception of spatial distribution in point distributions. Cartography and Geographic Information Science, 27: 51-64.

Sauer, C. O. 1956. The education of a geographer. Annals of the Association of American Geographers, 46: 287-368

Slocum, T.A., R.B. McMaster, F.C. Kessler, and H.H. Howard. 2005. Thematic Cartography and Geographic Visualization ( $2^{\text {nd }}$ ed.). Upper Saddle River: Pearson Prentice Hall.

Sowden, S., D. Stea, M.Blades, C. Spenser, and J.M.Blaut. 1996. Mapping abilities of four-year-old children in York, England. Journal of Geography, 95: 107-111.

Trifonoff, K.M. 1994. Using thematic maps in the early elementary grades. $\mathrm{PhD}$ dissertation: University of Kansas, Department of Geography.

United States Census Bureau. 2000. Census 2000 Data for the State of Oregon. Available at: www.census.gov. (last accessed 10 October 2006).

Verdi, M.P. and R.W. Kulhavy. 2002. Learning with maps and texts: An overview. Educational Psychology Review, 14: 27-46.

Vygotsky, L.S. 1978. Mind in society: The development of higher psychological functions. Cambridge: Harvard University Press.

Wiegand, P. 2003. School students' understanding of choropleth maps: Evidence from collaborative mapmaking using GIS. Jourmal of Geography, 102: 243242.

Young, J.E. 1994. Learning from Thematic Maps: Children's Cognitive Processing and the Integration of Mapped Information. $\mathrm{PhD}$ dissertation: University of Minnesota, Department of Education. 


\section{APPENDIX A: \\ MIDDLE SCHOOL TEXTBOOKS INCLUDED IN SURVEY}

Appleby, J., J.M. McPherson, and A. Brinkley. 2005. The American Joumey. New York: McGraw Hill Glencoe.

Appleby, J., J.M. McPherson, A. Brinkley, D.A. Ritchie, and A.S. Broussard. 2005. The American Republic to 1877. New York: McGraw Hill Glencoe.

Bednarz, S.W., M.C. Schug, I.M. Miyares, and C.S. White. 2005. World Cultures and Geography. Boston: Houghton Mifflin.

Boehm, R.G. 2003. Our World Today: People, Places, and Issues. Columbus: Glencoe/McGraw-Hill.

Boehm, R.G. 2005. The World and its People. Columbus: Glencoe/McGraw-Hill. Davidson, J.W. and M.B. Stoff. 2005. The American Nation Civil War to the Present. Upper Saddle River: Pearson Prentice Hall.

Garcia, J., D.M. Ogle, C.F. Ringer, J. Stevos, and W.D. Jordan. 2001. Creating America: $A$ History of the United States. Evanston: McDougal Littell.

Helgren, D.M., R.J. Sager, and A.S. Brooks. 2005. Western World: People, Places, and Change. Austin: Holt, Rinehart, and Winston.

Kracht, J.B. World Explorer: People, Places, and Cultures. Needham: Prentice Hall.

Pearson Prentice Hall. 2005. History of our World. Needham: Pearson Prentice Hall.

Spielvogel, J.J. 2006. World History: Joumey Across Time. New York: McGraw Hill Glencoe.

Stuckey, S. and L.K. Salvucci. 2005. Call to Freedom: The Beginnings to 1914. Austin: Holt, Rinehart, and Winston. 


\section{APPENDIX B: \\ MAP CREATION METHODOLOGY}

\section{Data}

The Oregon county population dataset that was used in each map was downloaded from the United States Census Bureau (2000). The summary statistics for Oregon's population by county is described in Table B1.

Table B1. Summary statistics of population in the 36 Oregon counties. The results illustrate the wide range of county population values.

\begin{tabular}{rr} 
Statistic & $\begin{array}{c}\text { People per } \\
\text { County }\end{array}$ \\
\hline Min: & 1,547 \\
Max: & 660,486 \\
Range: & 658,939 \\
Mean: & 95,039 \\
Median: & 39,595 \\
Mode: & None \\
\hline
\end{tabular}

The data has a large range, and similarly to much geographic data, is positively skewed, meaning that there are more counties that have low to medium population values and very few that have extremely high values. This poses some design issues that have to be addressed differently by each symbolization method. 


\section{Dot Map}

Dot maps have often been referred to as the "simplest" point-symbol map because the placement of dots reduces abstractness of symbols (Jenks 1974). Although it may appear to be a "simple" map, there are many design issues including symbol size, value, placement, and visual hierarchy that have to be addressed by the cartographer to produce a clear map for the reader.

To show the Oregon population data, I chose one dot to represent 500 people based on the guideline that a dot value should be chosen so that the area with the fewest data counts is represented by at least 2 to 3 symbols (Mackay 1949, Dent 1996). Wheeler County only has 1,547 people. One third of that value is 500 , which happens to be an easy, round number that people understand.

Once I determined its value, I then had to decide where to place the dots within the county boundaries. Symbols in dot maps must be placed so they represent locations where phenomena most likely occur (Dent 1996). I chose to use the forest shapefile from the Oregon Geospatial Clearinghouse to provide a rough estimate of where dots should not be concentrated. Then, I used the city boundary shapefile, also from the Oregon Geospatial Clearinghouse, to aid in dot placement and concentration.

Once the dots were placed, I had to choose a dot size that would show the densest areas' dots beginning to coalesce (Mackay 1949, Dent 1996). This guideline allows the dots of the densest areas to be more tightly-packed than in sparsely populated areas, suggesting a high density. Therefore, I used 
Multnomah County's distribution - the most densely populated county in the state- to aid in determining the size of the dot. As Figure B1 illustrates, Multnomah County and surrounding areas have dots that are so closely placed, they appear to be almost a solid block, suggesting a very high density. If the dots would have been larger, it would be impossible to distinguish individual dots, defeating the purpose of the dot map. If the dots would have been smaller, there would be too much space between dots in the densest areas and people would perceive the area as lower density. Also, it would make the single dots in very low density areas difficult to detect.
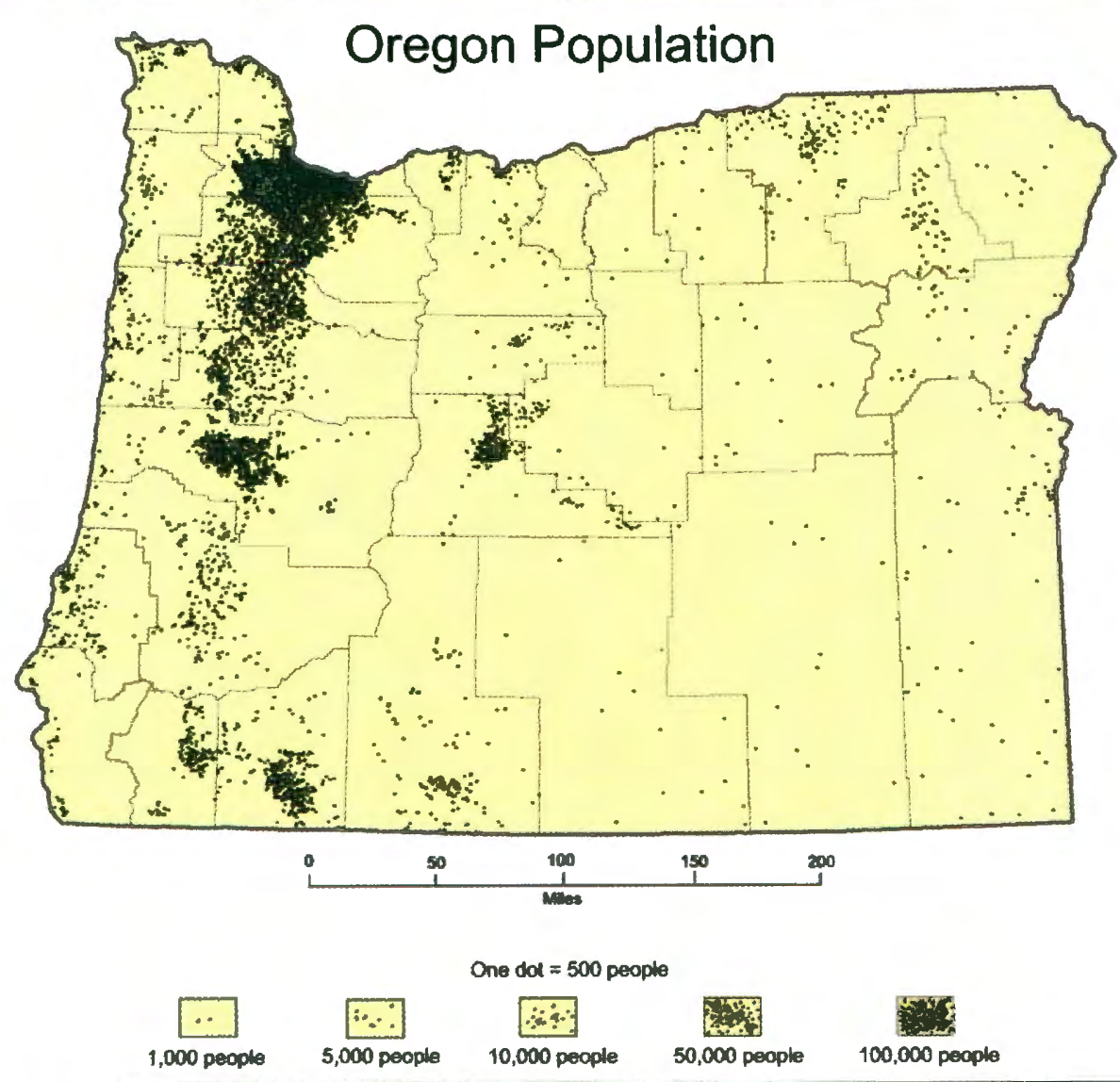

Figure B1. The dot map used for the study 
One common method cartographers employ to deal with outlier counties such as Multnomah County is to use a graduated circle to represent the dense enumeration unit or high-density urban areas. However, because this thesis aims to determine differences between symbols, it is illogical to use two symbols on one map. The dot size seen in Figure Bl shows a selected dot size effective to allow the eye to detect areas of high densities and areas of low densities.

The last consideration of dot maps is figure-ground relationships. To have the points become the figure, or the object that stands out as most important, the points should strongly contrast with the background information (Slocum et al. 2005). For this reason, I chose black dots on a light yellow background with $30 \%$ gray county outlines to provide contrast.

\section{Graduated Circle Map}

Graduated circle maps have been used by cartographers because of the ease of converting numerical data to circle symbols where the circle area represents the respective value (Slocum et al. 2005). Additionally, circles are "more visually stable than other symbol forms and thus cause little eye wandering" (Dent 1996, 167). The scaling of circles may involve making the areas directly proportional to the value represented or making the areas psychologically-scaled according to a perceptual scaling factor. Research shows that people underestimate the size of large circles, so some have suggested a method of perceptual scaling to adjust for the underestimation of circles 
(Flannery 1971). Critics of Flannery argue that perceptual scaling cannot accurately account for those people who are poor estimators and as a result, negatively affect those who do estimate well (Griffin 1985, Griffin 1990). When perceptual scaling is used, there is more variance in estimation than when mathematical scaling is used, even though everyone underestimates.

Additionally, Chang (1980) found that using multiple values in the legend generally spanning the range of circle sizes appearing in the map aided readers in estimating circle size as compared to just including one circle size in the legend. Based on this research, I am choosing to scale the circles directly proportional to the data values and including five circle sizes in the legend.

Additionally, the size of circles should be chosen so that overlap of circles should be moderate (Dent 1996). If the circles are too large, the map appears crowded. Conversely, if the circles are too small, the map appears empty. The base circle size was chosen so that the Multnomah, Washington, and Clackamas County area circles did not overlap excessively. I placed circles at the centroid of each county (Slocum et al. 2005).

With regard to figure-ground relationships, opaque symbols are useful to create the greatest visual contrast (Griffin 1990). For this reason, I chose black opaque symbols on a light yellow background.

Another factor to consider in graduated circle maps is surrounding circle sizes affecting the perception of adjacent circles (Gilmartin 1981). This phenomena is generally known as the Ebbinghaus illusion (Slocum et. al 2005). 
Both Gilmartin (1981) and Dent (1996) note that there is little that can be done to correct for this problem, but just be aware that it exists. The final graduated circle map can be found in Figure B2.

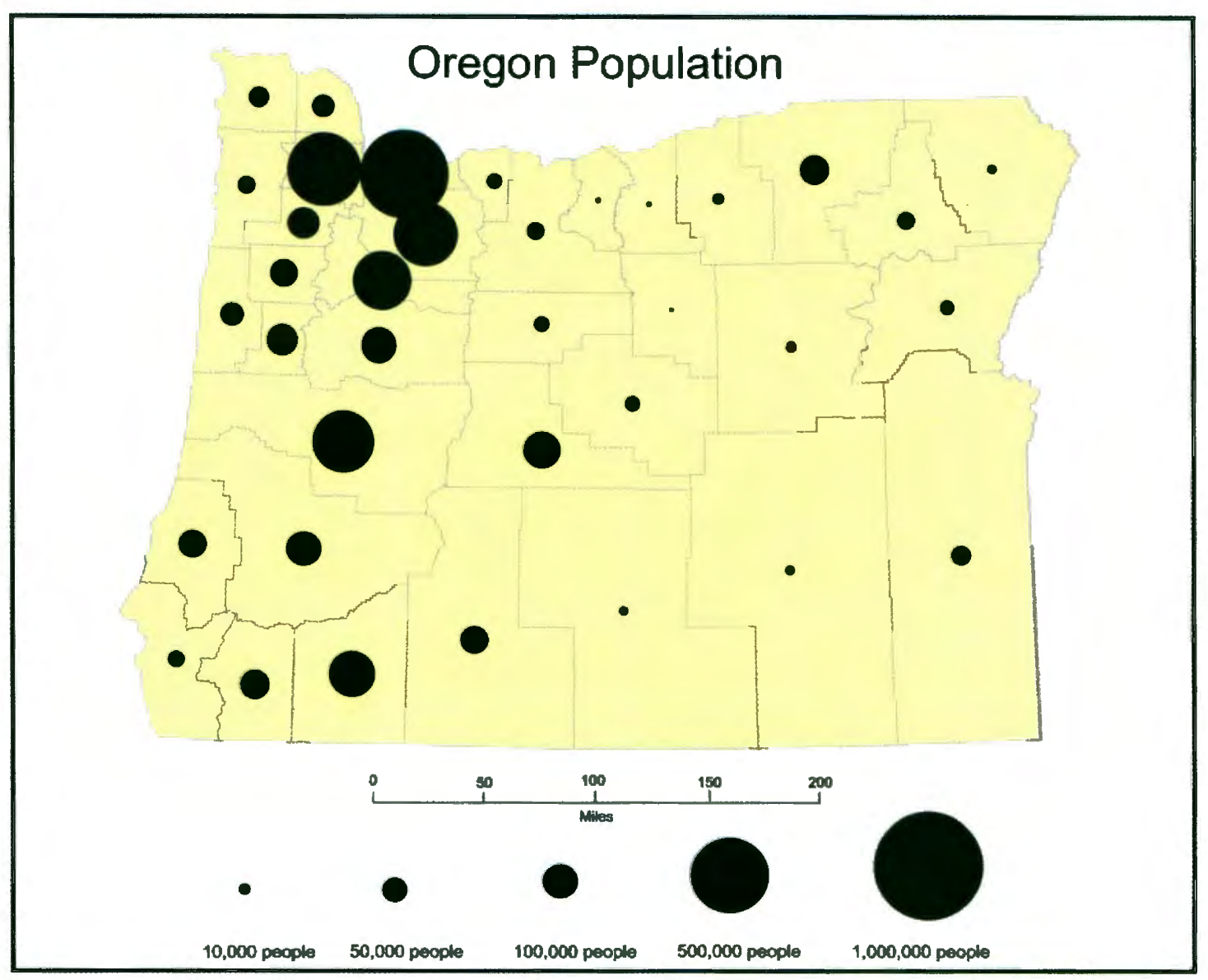

Figure B2. The graduated circle map used in the survey.

\section{Choropleth Map}

The choropleth map is probably the most commonly employed map type in everyday use for population data because it is very easily constructed. It divides a map into enumeration units, in this case counties, and shades each unit with a tone representing a standardized value. In Figure B3, I chose to 
standardize the county population values to the commonly used people per square mile. To obtain this, each county's population is divided by the total county land area to produce the range of values seen in Table B1. These values are then grouped into classes. The optimal number of classes is between four and six (Dent 1996). I chose five classes so that there is a clear "middle" value and because Wiegand (2003) found students tended to class data into five categories when given the task to class data on choropleth maps. I chose Jenks optimal classification method, a widely recommended approach (MacEachren 1995, Slocum et. al 2005).

The last concern for choropleth maps is an effective color scheme. Cartographic literature almost universally suggests not using a sequence of hues, since color differences are usually associated with qualitative differences. Instead, it is recommended that a sequential scheme of tones be used where a hue is darkened as values become higher and hue and saturation are held relatively constant. Therefore, the lightest color represents the lowest value (or groups of values) and the darkest color represents the highest value (s) (Brewer 1994). Brewer also suggests that saturation values can be darkened to create clear contrast between all classes. I used a color sequence from Colorbrewer (2002) and chose orange because it is a common color seen in textbooks to symbolize population. 


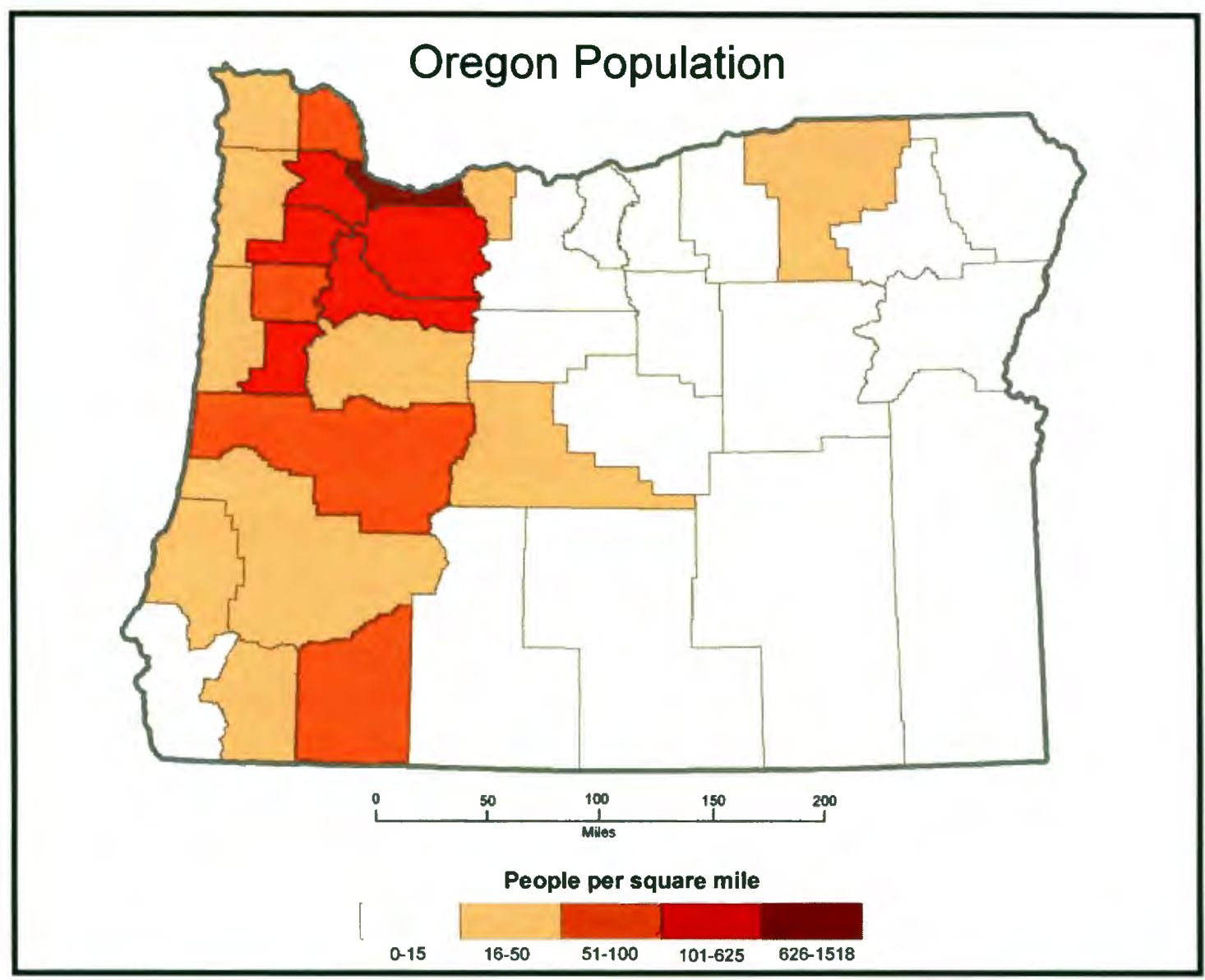

Figure B3. The choropleth map used in the surveys. 


\section{APPENDIX C: \\ MAP SURVEYS \\ (pages of surveys have been reformatted \\ to fit page margin requirements)}




\section{Choropleth Map Survey (4 pages)}

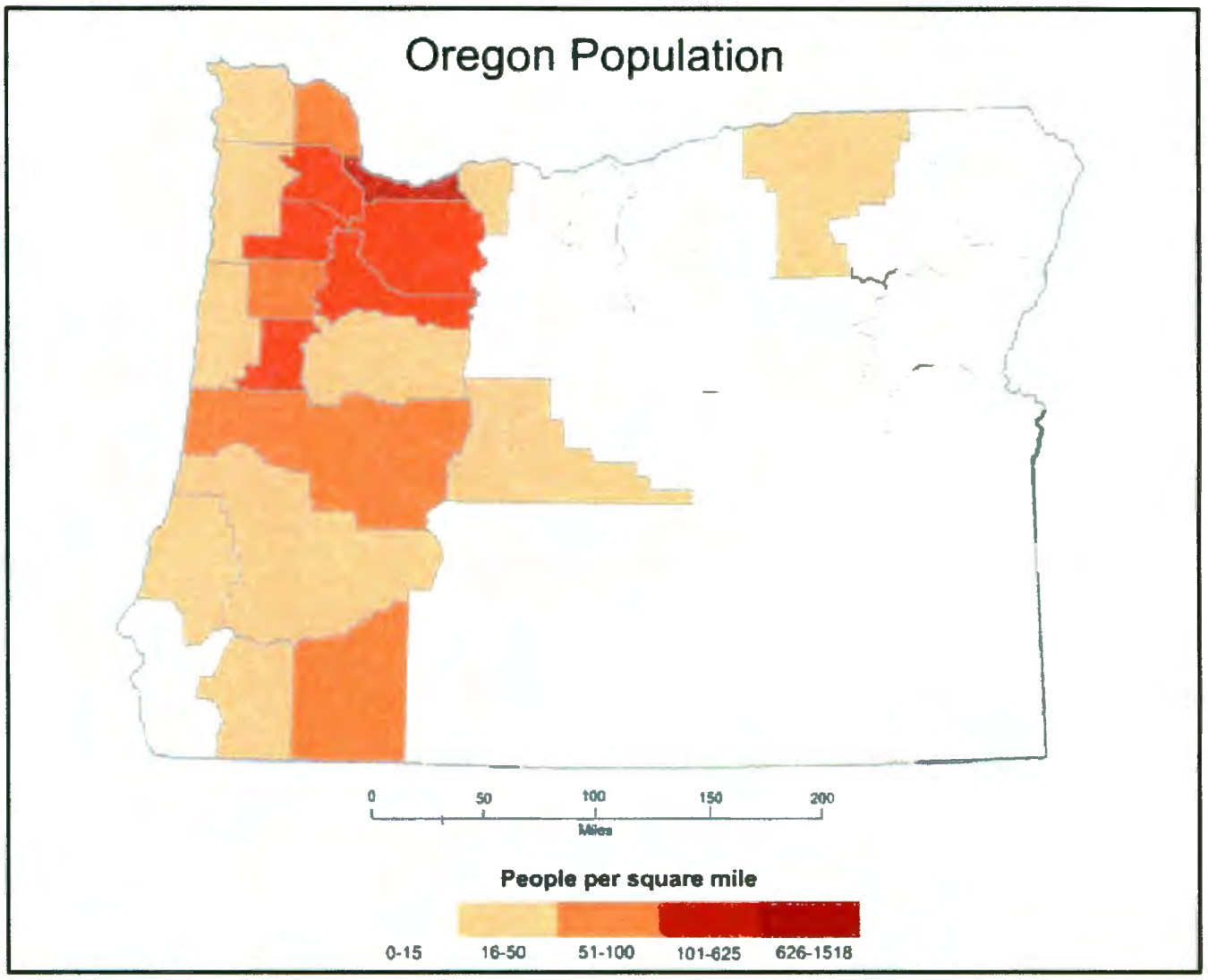

1. On the map at the right there is a highlighted county. Find the same county in the map above. According to the map above, how many people would you estimate live in that county?

2. Mark an $X$ in one county on the map at the top of the page that you think has a city with many people living in it. There may be more than one correct answer, but only put an $\mathrm{X}$ in one county. 


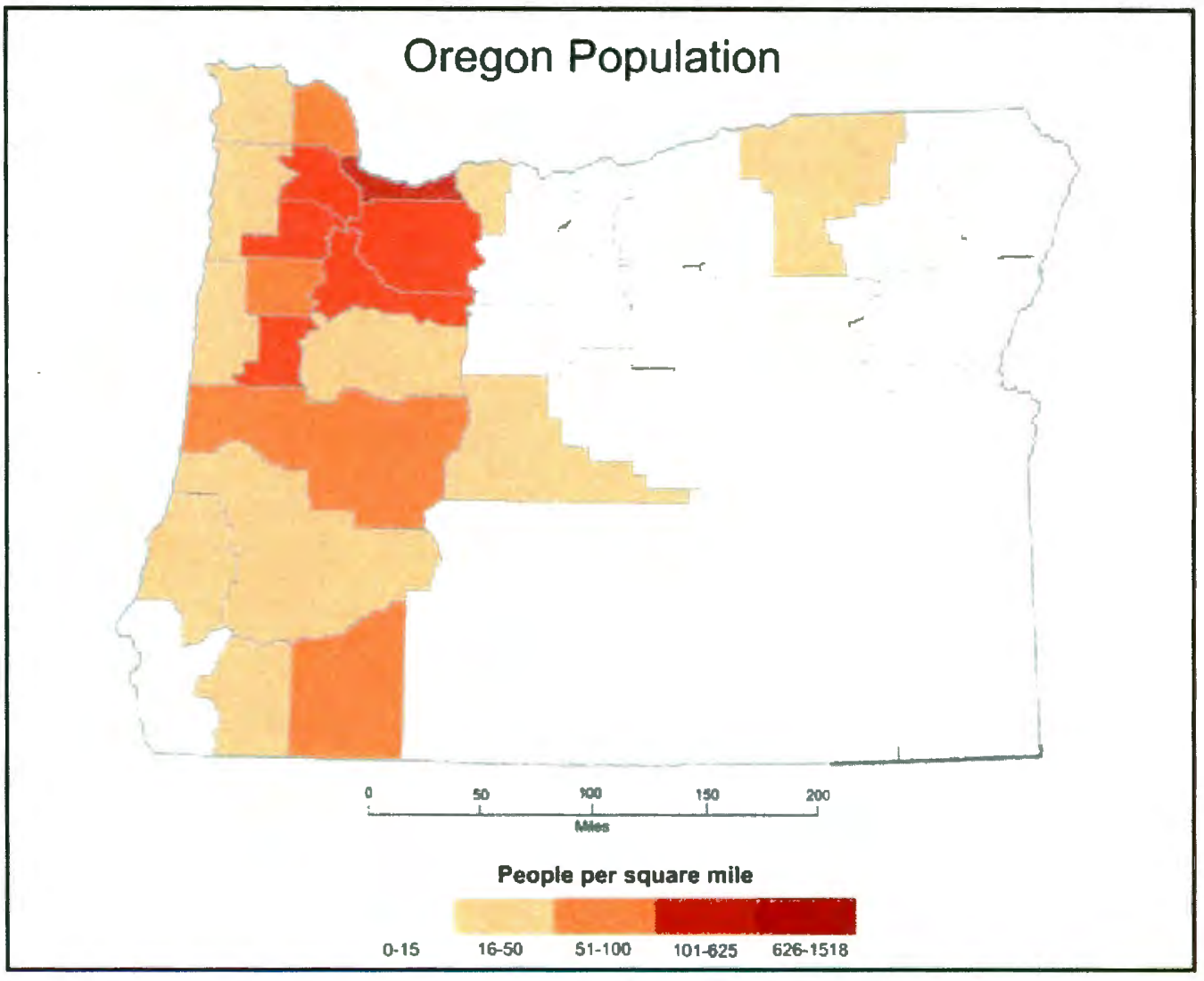

3. On the map at the right, look at the highlighted County A and County B. Find these counties in the map above. Looking at the map above, which A B county has more people: county $\mathrm{A}$ or county B?

4. Geographers say that a region is an area that includes more than one county. A region does not have to follow county borders. Many people see regions of high, medium, and low population on the map at the top of the page. On the map at the top of the page, draw lines around the regions that you think are high, medium, and low and label each with the word "High," "Medium," or "Low." When you get done, every place in Oregon should be in a high, medium, or low region. 


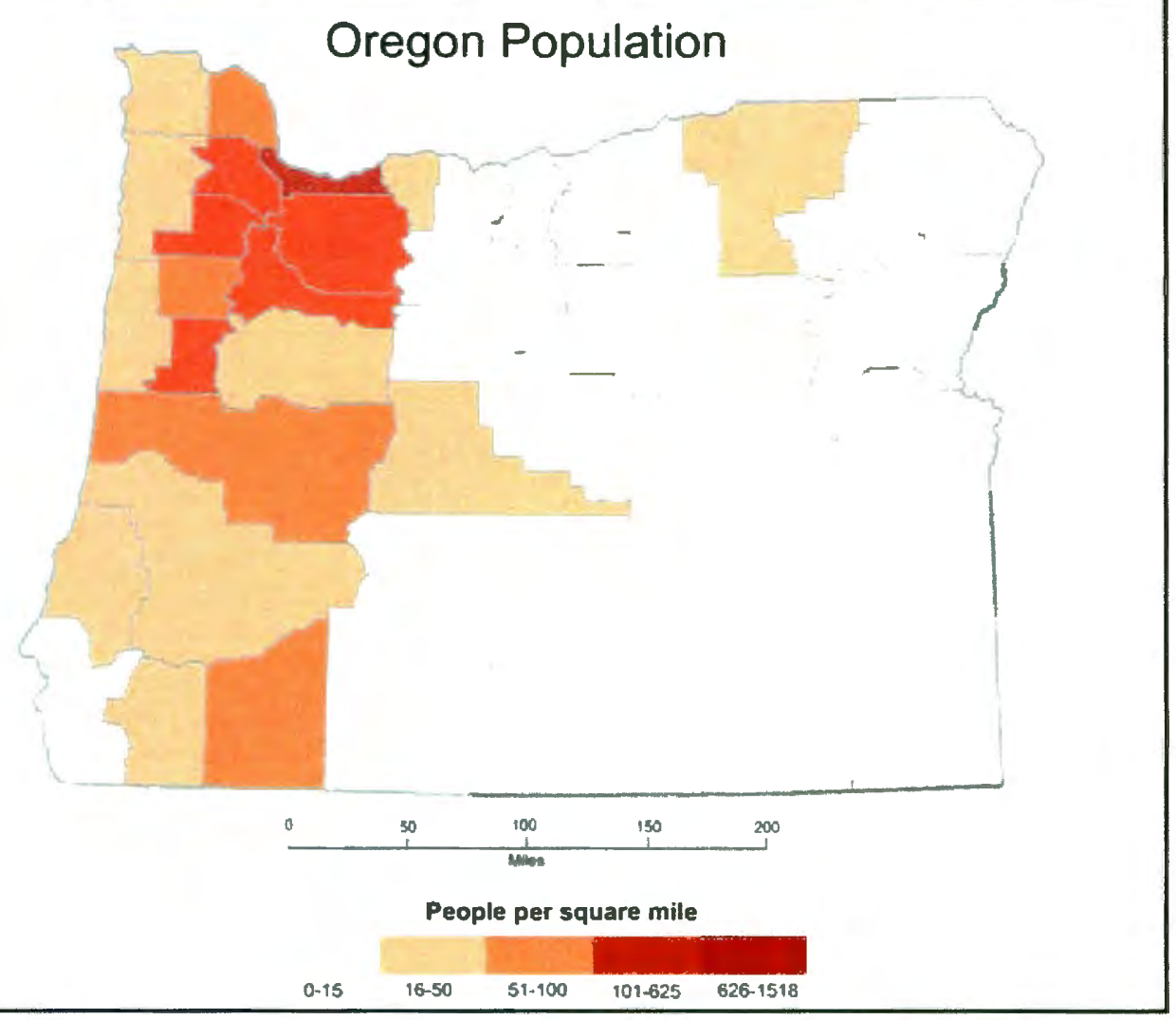

5. Imagine that you are a reporter from another state and you wanted to write a story about where people live in Oregon. You look at this map as a starting point and learn where people live in Oregon. However, looking at the map and where it shows people living, you begin to think of other questions about Oregon's population. If you can find answers to these questions, it will help you write an informative story on Oregon population. Before you do any more research, you need to have questions that you will try to find answers to. So, think of some questions you have about Oregon's population by looking at the map.

In the space below, write down 2 questions that the map makes you ask about where people live in Oregon that will be important to writing your story. Do not write the answers, just the questions. 
6. The whole map is shaded a color, but people don't live in every square mile in Oregon because there are forests, lakes, and rivers where people cannot live. But, the map is still correct. Explain how:

7. How old are you?

8. Are you a boy or a girl?

9. What words would you use to describe this exercise? Confusing? Interesting? Hard? Easy? Fun? Or something else? Please explain your answer. 
Dot Map Survey (4 pages)

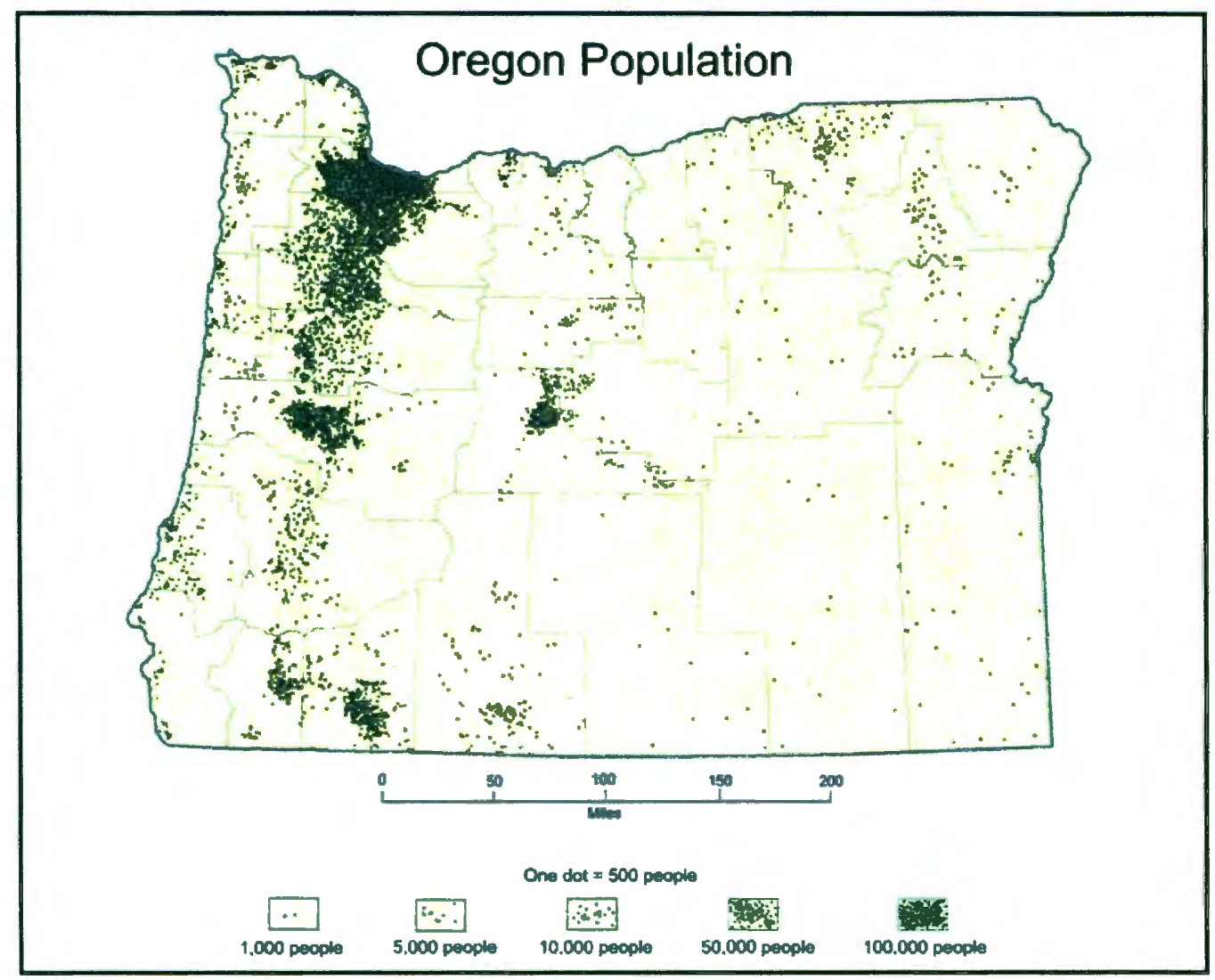

1. On the map at the right there is a highlighted county. Find the same county in the map above. According to the map above, how many people would you estimate live in that county?

2. Mark an $X$ in one county on the map at the top of the page that you think has a city with many people living in it. There may be more than one correct answer, but only put an X in one county. 


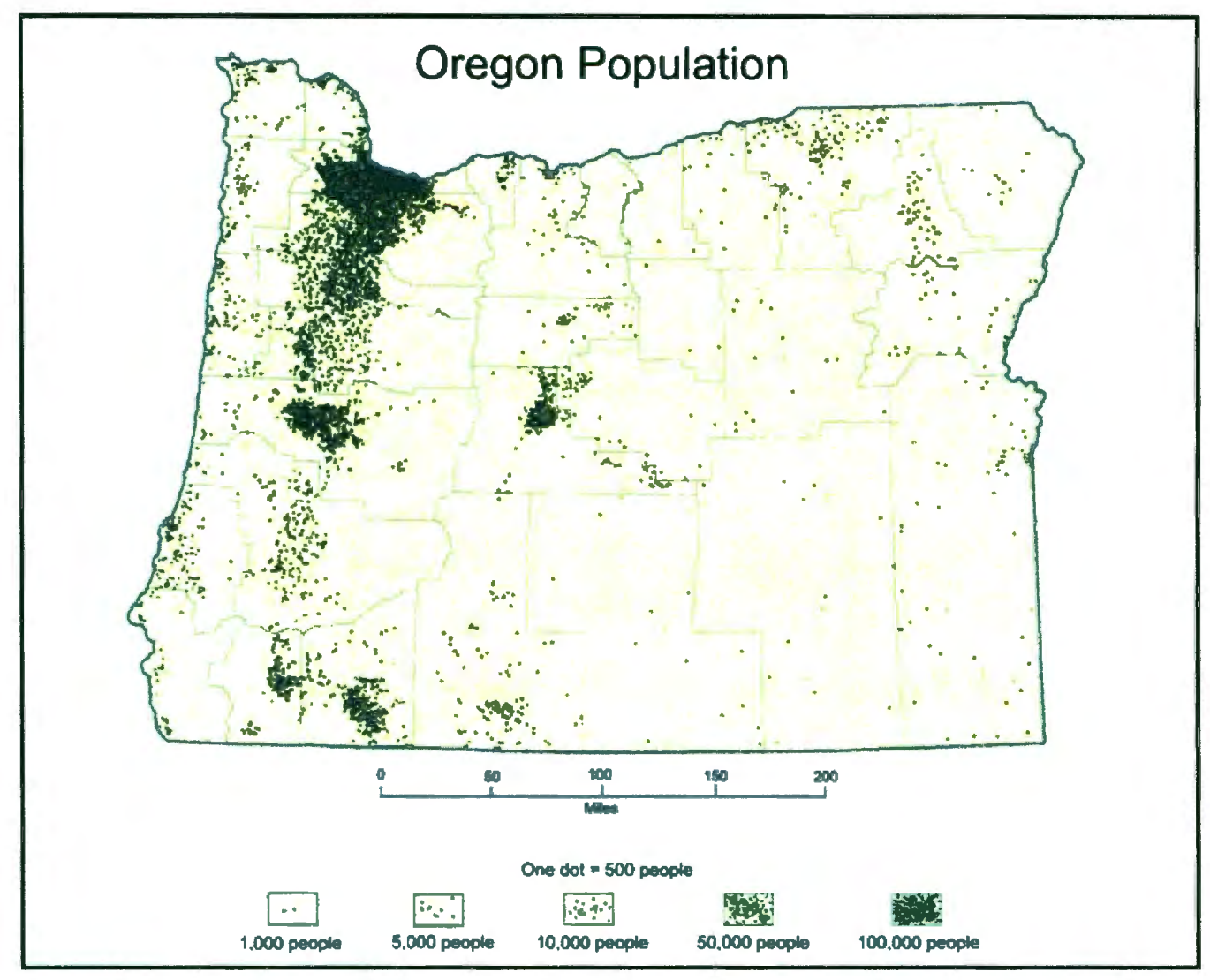

3. On the map at the right, look at the highlighted County A and County B. Find these counties in the map above. Looking at the map above, which county has more people: county A or county B?

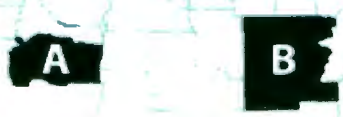

4. Geographers say that a region is an area that includes more than one county. A region does not have to follow county borders. Many people see regions of high, medium, and low population on the map at the top of the page. On the map at the top of the page, draw lines around the regions that you think are high, medium, and low and label each with the word "High," "Medium," or "Low." When you get done, every place in Oregon should be in a high, medium, or low region. 


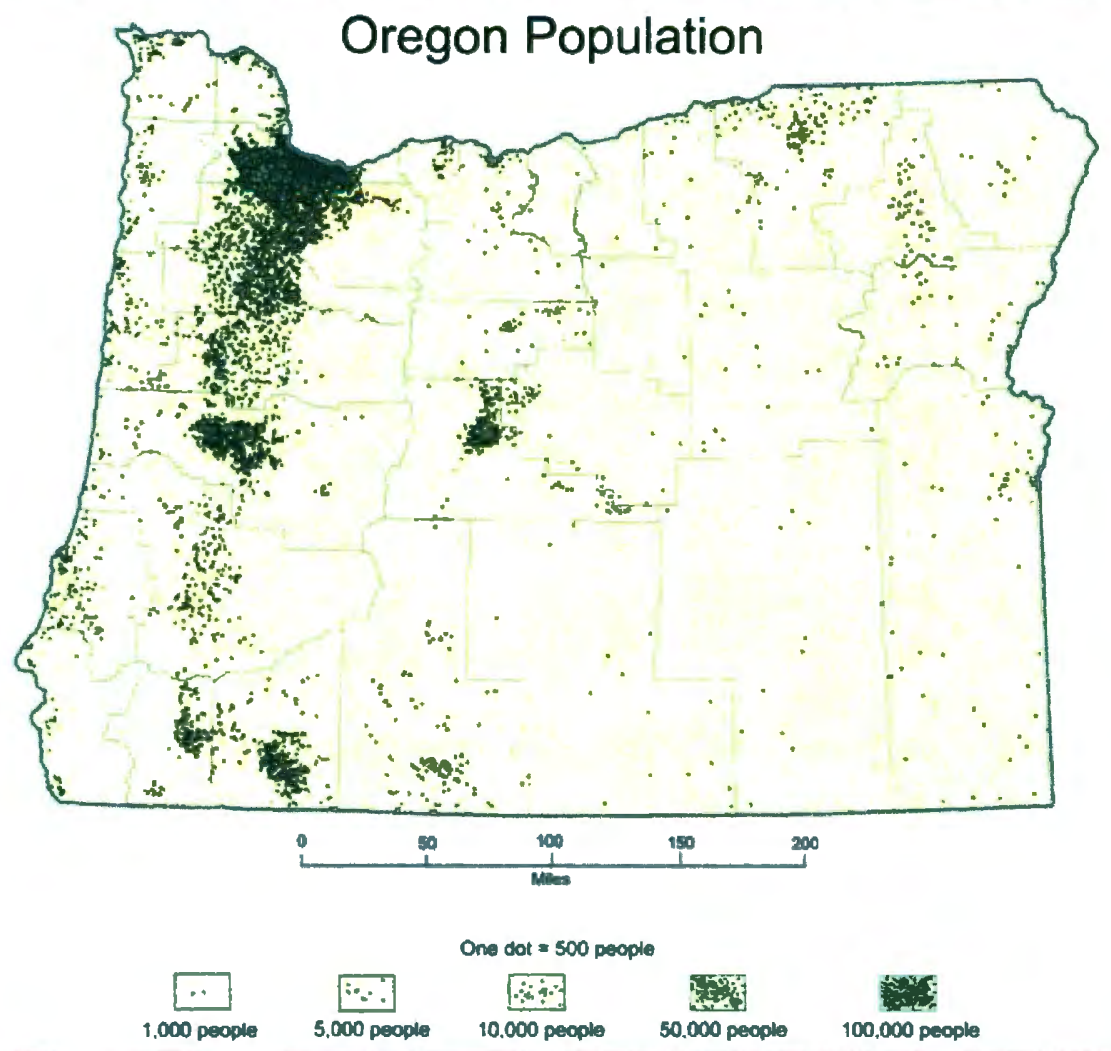

5. Imagine that you are a reporter from another state and you wanted to write a story about where people live in Oregon. You look at this map as a starting point and learn where people live in Oregon. However, looking at the map and where it shows people living, you begin to think of other questions about Oregon's population. If you can find answers to these questions, it will help you write an informative story on Oregon population. Before you do any more research, you need to have questions that you will try to find answers to. So, think of some questions you have about Oregon's population by looking at the map.

In the space below, write down 2 questions that the map makes you ask about where people live in Oregon that will be important to writing your story. Do not write the answers, just the questions. 
6. People live in parts of Oregon where there are no dots on the map, but the map is still correct. How can this be?

7. How old are you?

8. Are you a boy or a girl?

9. What words would you use to describe this exercise? Confusing? Interesting? Hard? Easy? Fun? Or something else? Please explain your answer. 
Graduated Circle Map Survey (4 pages)

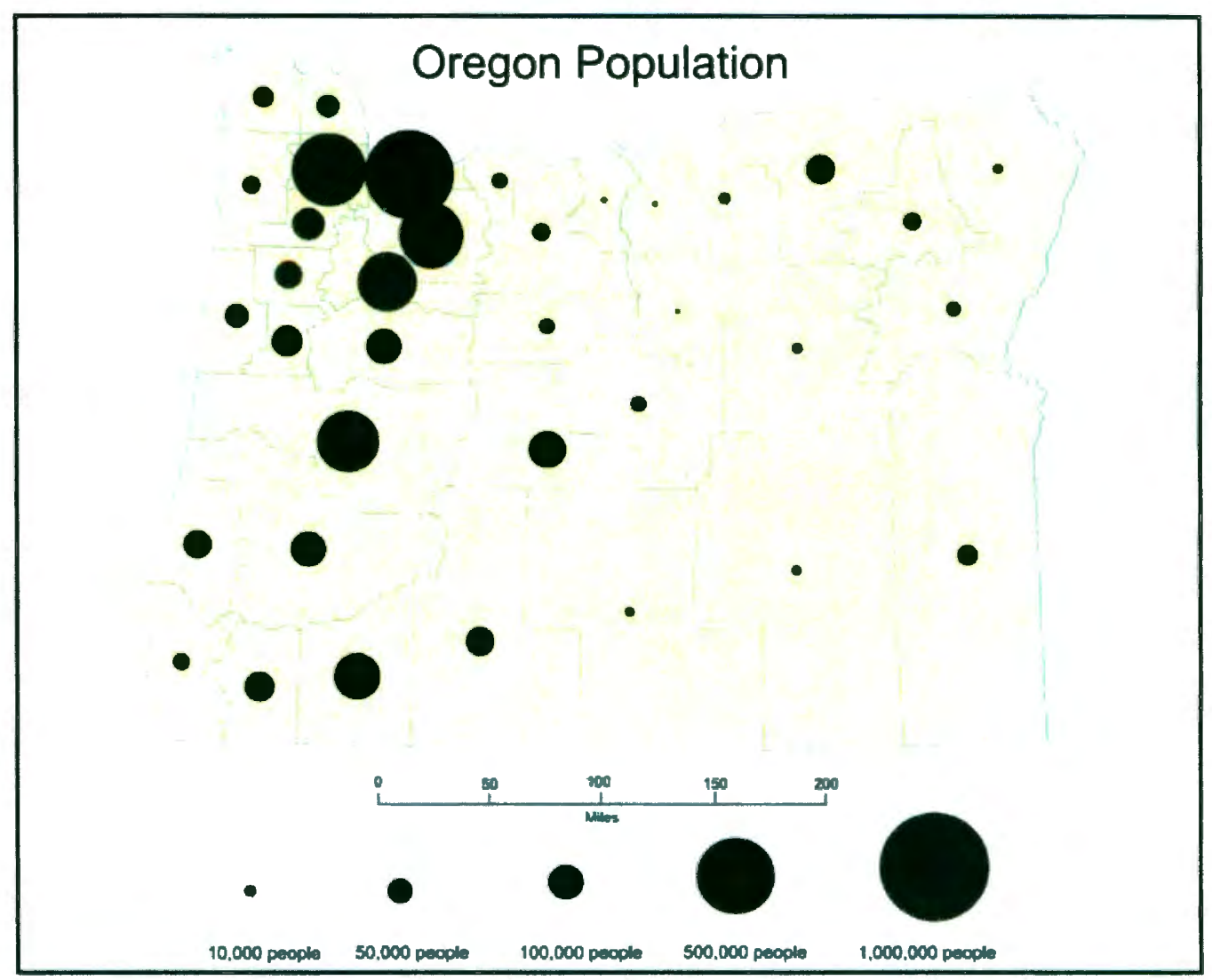

1. On the map at the right there is a highlighted county. Find the same county in the map above. According to the map above, how many people would you estimate live in that county?

2. Mark an $X$ in one county on the map at the top of the page that you think has a city with many people living in it. There may be more than one correct answer, but only put an $\mathrm{X}$ in one county. 


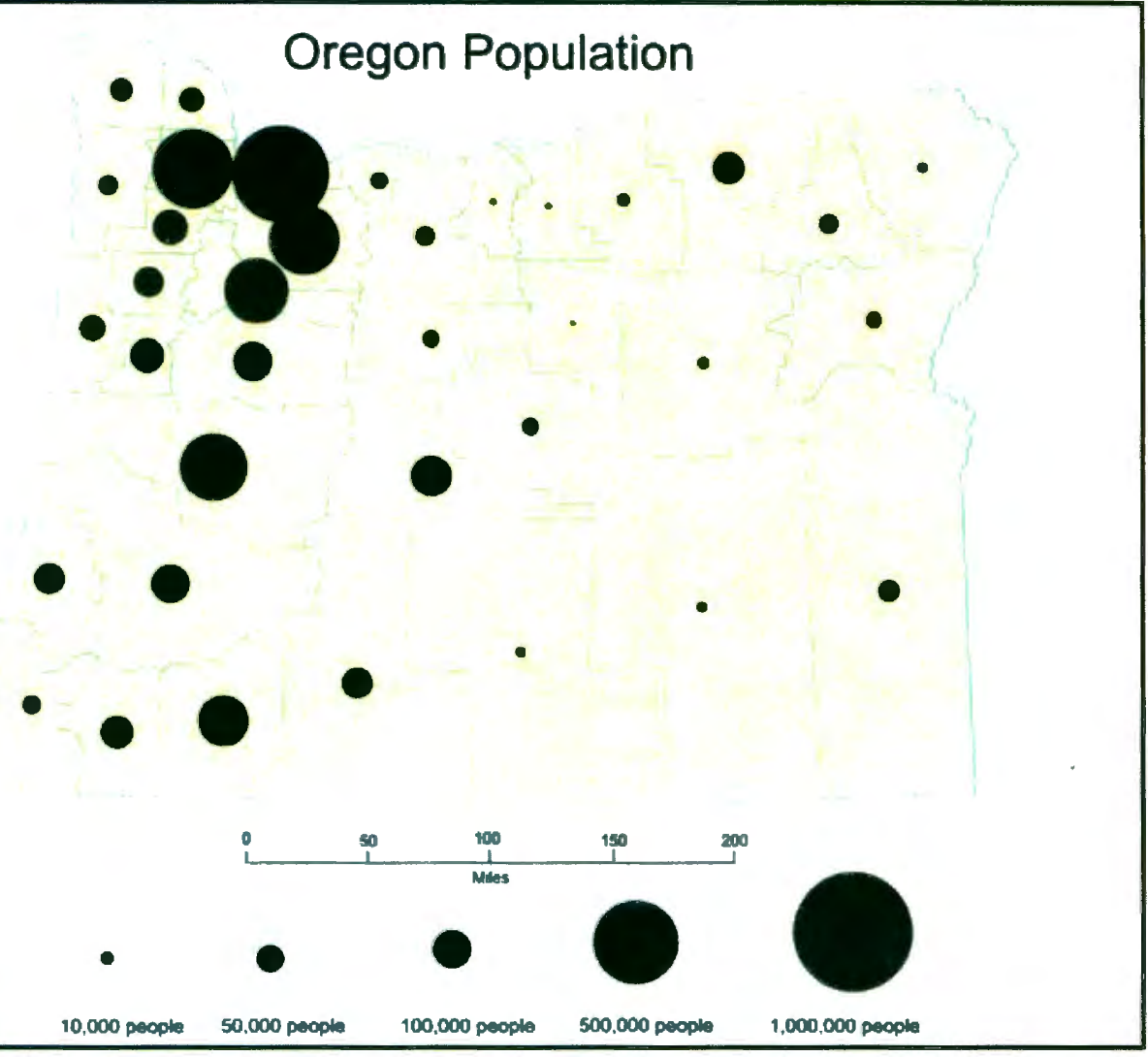

3. On the map at the right, look at the highlighted County A and County B. Find these counties in the map above. Looking at the map above, which A $\quad$ B county has more people: county A or county B?

4. Geographers say that a region is an area that includes more than one county. A region does not have to follow county borders. Many people see regions of high, medium, and low population on the map at the top of the page. On the map at the top of the page, draw lines around the regions that you think are high, medium, and low and label each with the word "High," "Medium," or "Low." When you get done, every place in Oregon should be in a high, medium, or low region. 


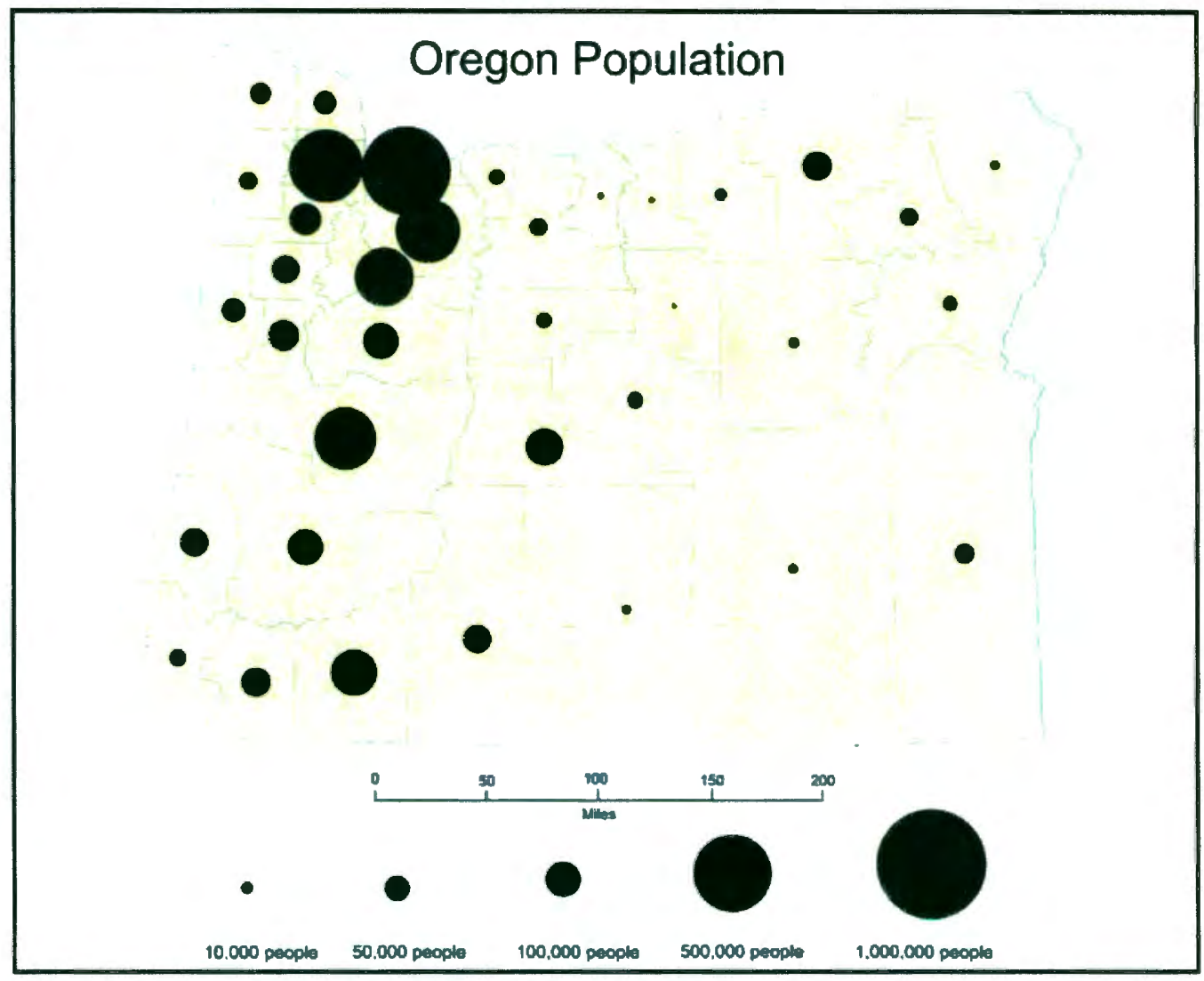

5. Imagine that you are a reporter from another state and you wanted to write a story about where people live in Oregon. You look at this map as a starting point and learn where people live in Oregon. However, looking at the map and where it shows people living, you begin to think of other questions about Oregon's population. If you can find answers to these questions, it will help you write an informative story on Oregon population. Before you do any more research, you need to have questions that you will try to find answers to. So, think of some questions you have about Oregon's population by looking at the map.

In the space below, write down 2 questions that the map makes you ask about where people live in Oregon that will be important to writing your story. Do not write the answers, just the questions. 
6. People live in areas in Oregon that aren't covered by the circle in each county, but the map is still correct. How can this be?

7. How old are you?

8. Are you a boy or a girl?

9. What words would you use to describe this exercise? Confusing? Interesting? Hard? Easy? Fun? Or something else? Please explain your answer. 


\section{APPENDIX D:}

\section{HUMAN RESOURCES APPROVAL}

Hurnen Subjects Reseanch Review Committee

Past Oince Bax 751

Portund, Orecon $97207-0751$

$503-725-4288$ ted

$503-725-3416 \mathrm{tmx}$

narrefllists.pax.edu

December 20, 2006

To: Stephanie Gaspen

From: Nency Koroloff, HSRRC Chiit

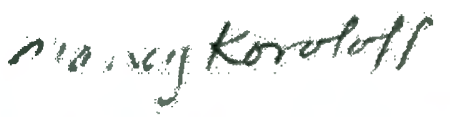

Re: HSRRC waived review of your upplicution tided "Which Thematic Maps Are Practical for Educational Purposes? A Study with Middle Sehool Students" (HSRRC Proposal \# 06346).

Dear Stephanic,

Your proposal is exempt from further Human Subjects Research Review Commitwee review, and you may proceed with the study.

Even with the exemption above, it wras necessary by University policy for you to notify this Committee of the proposed research, and we appreciacte your timely attention to this matter. If you make changes in the research protocol, the Committee muat be notified in writing and changes must be approved before being implemented.

If you have questions or concerns, please conmet the HSRRC in the Office of Research and Sponsored Projects (ORSP), (503) 725-4288, 111 Cramer Hall.

\section{Ce Joseph Ponesky}




\section{APPENDIX E:}

\section{SCRIPT}

Hello everyone. Thanks for having us today! My name is Steph and this is my friend Jenny. We are both geographers at Portland State University. Today we need your help by doing a short little exercise with us about maps. You will be helping me tremendously. Without you, I won't be able to do my homework. And you all know how it feels when you can't get your homework done, it's not a good feeling, is it?

If you all agree to help me out, you won't have to worry about this counting for your grade because the things you write down won't be recorded by Ms. In fact, you aren't even going to write your name on it, so we won't know which paper is even yours. Also, you don't have to worry about putting down a "wrong" answer because most of the questions don't have a wrong answer. I'm just interested in what you think about the maps. So, it should be a fun exercise that will help you learn a little about maps and about Oregon population.

Does anyone not want to participate? Just let me know and you won't have to, it's ok.

Ok, good. Jenny is going to hand you a packet that you will keep face down. Do not flip it over until we tell you to so we can go over the instructions first. Also, please do not write your name on it because like I said before, we don't want to know which paper is yours when you are done.

So, all of your packets have a map showing Oregon population. When you get your packet, there are 4 pages with a total of 9 simple questions for you to answer about the map. You can write your answers directly on the paper. If at any time you have a question about anything in the exercise, raise your hand and Jenny or I will come and help you out.

Remember, if you have any questions during the exercise, please raise your hand and we'll come and help you. If you still can't figure out the question after we help you, it may be that the question is just too hard. And it's ok, because some of the questions may be pretty hard, so it's ok to put that you don't know and that will help me just as much as an answer you get. But, try to do your best at answering the questions because I will be using what you write to help me learn about maps, so your answers are really important to me. And remember, if you need help or are confused, just raise your hand.

When you are all done, turn your packet over and raise your hand so we can collect your packet.

Does anyone have any questions so far? Does anyone need me to repeat anything? Ok, then you can all turn your packet over and begin the exercise. 